Supporting Information for

\title{
Organocatalyzed Photoredox Radical Ring-Opening Polymerization of Functionalized Vinylcyclopropanes
}

\author{
Dian-Feng Chen, Simone Bernsten, and Garret M. Miyake* \\ Department of Chemistry, Colorado State University, Fort Collins, CO 80523, United \\ States
}

\section{Table of Contents}

1. Materials and Methods................................................................................... 2

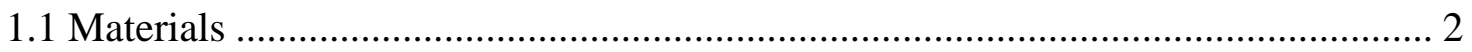

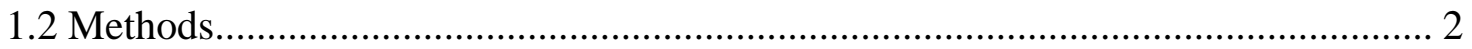

2. Synthesis of Functionalized Vinylcyclopropanes ............................................. 4

3. Impact of Additives On Organocatalyzed Photoreodx Radical Ring-Opening

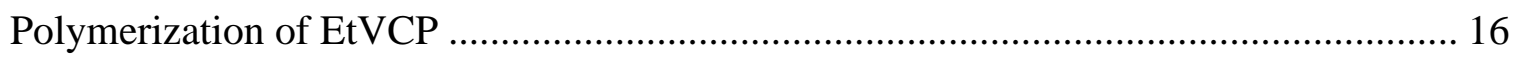

4. Polymerization of CNVCP, EtVCP-CN and EtVCP-NHPh ................................ 17

4.1 General Experimental Procedures............................................................. 17

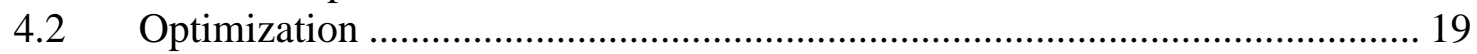

$4.3{ }^{1} \mathrm{H}-\mathrm{NMR}$ Spectrum of Poly(EtVCP-CN) and Poly(EtVCP-NHPh) ................. 20

4.4 GPC Traces of Poly(EtVCP-CN) and Poly(EtVCP-NHPh) .......................... 22

4.5 DCS and TGA Analysis of Poly(EtVCP-CN) and Poly(EtVCP-NHPh) ......... 23

5. Polymerization of Natural Product-Derived Vinycylopropanes............................. 26

$5.1 \quad$ General Experimental Procedures.............................................................. 26

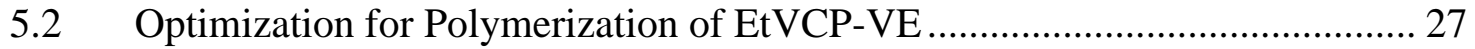

5.3 Kinetic Study and Pulsed-Irradiation Experiment ....................................... 28

5.4 Chain-Extension Experiment ....................................................................... 30

$5.5{ }^{1} \mathrm{H}-\mathrm{NMR}$ Spectrum of Natural Product-Derived Poly(vinylcyclopropanes) .... 31

5.6 GPC Traces of Natural Product-Derived Poly(vinylcyclopropanes) ................. 36

5.7 DSC and TGA Curves of Natural Product-Derived Poly(vinylcyclopropanes) 40

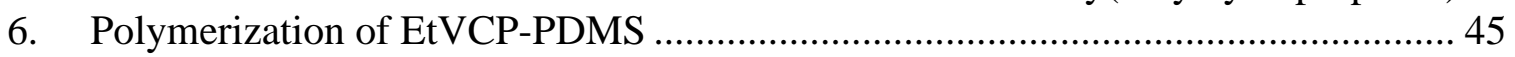

6.1 General Experimental Procedure ..................................................................... 45

$6.2{ }^{1} \mathrm{H}-\mathrm{NMR}$ Spectrum of Brush Poly(EtVCP-PDMS) .......................................... 46

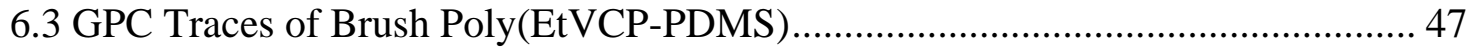

6.4 DSC and TGA Curves of Brush Poly(EtVCP-PDMS) ...................................... 48

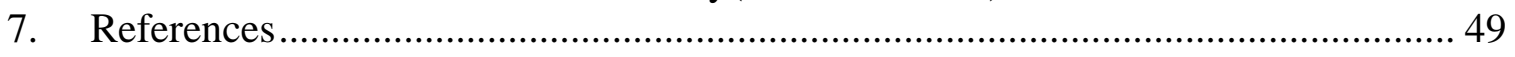




\section{Materials and Methods}

\subsection{Materials}

Phenoxazine was purchased from Beantown Chemical. Trans-1,4-dibromo-2-butene, diethyl malonate and 6-bromohexanoic acid were purchased from TCI America. Dehydroabietic acid was purchased from Astatech. Deuterated chloroform was purchased from Cambridge Isotope Laboratories. All other chemicals and anhydrous solvents were purchased from Sigma-Aldrich.

Ethyl $\alpha$-bromophenylacetate (EBP), methyl 2-bromopropionate (M2BP) and diethyl 2-bromo-2-methylmalonate (DBMM) were purified by vacuum distillation, degassed by three freeze-pump-thaw cycles and stored at $-34{ }^{\circ} \mathrm{C}$ in the freezer of a nitrogen-filled glovebox. All alkyl halides were allowed to warm to room temperature before use.

Photocatalysts (PCs) N,N-diaryl dihydrophenazines 1-2, ${ }^{1}$ 3,7-di(4-biphenyl)-Nnaphthylphenoxazine $3{ }^{2}$ and EtVCP-C2 ${ }^{3}$ were synthesized according to our previous reports.

\subsection{Methods}

Thin layer chromatography was performed on EMD Milipore silica gel 60 F254 glass plates. Column chromatography was performed on SiliCycle®SilicaFlash ${ }^{\circledR}$ P60, 40-63 $\mu \mathrm{m}$, 60A. Visualization was accomplished with silica-I2.

White-light LED beaker: a $45 \mathrm{~cm}$ of double density white LEDs (Creative Lighting Solutions, item no. CL-FRS1210-5M-12V-WH, 18 W/5 meters) was wrapped around the inside of a $400 \mathrm{~mL}$ beaker, and powered by a 12VDC power Supply (2.1A Commercial, 25W Power Supply by Creative Lighting Solutions LLC). The wattage of this setup is $\sim 1.6$ W.

The $34 \mathrm{~W}$ blue LED (HP150, 1.5 A, 34W) was purchased from Kessil.

${ }^{1} \mathrm{H}$ and ${ }^{13} \mathrm{C}$ spectra were recorded on a Bruker $400 \mathrm{~Hz}\left(100 \mathrm{~Hz}\right.$ for $\left.{ }^{13} \mathrm{C}\right)$ spectrometer at ambient temperature. All NMR spectra are referenced to the residual solvent $\left(\mathrm{CHCl}_{3}\right)$ signal.

Analysis of polymer molecular weight and dispersity was performed using gel permeation chromatography (GPC) coupled with multi-angle light scattering (MALS), using an Agilent HPLC fitted with one guard column and three PLgel $5 \mu \mathrm{m}$ MIXED-C gel 
permeation columns in series. The detectors were a Wyatt Technology TrEX differential refractometer and a Wyatt Technology mini DAWN TREOS light scatter detector. The solvent used was THF with a flow rate of $1.0 \mathrm{~mL} /$ minute.

Glass transition temperatures $\left(T_{\mathrm{g}}\right)$ and melting point $\left(T_{\mathrm{m}}\right)$ of the polymers were measured by differential scanning calorimetry (DSC) on a DSC 2500 (TA Instrument) at a rate of $10{ }^{\circ} \mathrm{C} / \mathrm{min}$. $T_{\mathrm{g}}$ values reported were from the second heating scan (from -80 to $200{ }^{\circ} \mathrm{C}$, isothermed for $1 \mathrm{~min}$ ) after the thermal history (the first scan) being removed. Decomposition temperatures $\left(T_{\mathrm{d}}\right)$ at $10 \%$ of weight loss and maximum rate decomposition temperatures ( $\left.T_{\max }\right)$ of the polymers were measured by thermal gravimetric analysis (TGA) on a Q50 TGA Analyzer (TA Instrument), by heating the polymer samples from 25 to $700{ }^{\circ} \mathrm{C}$ at the rate of $10^{\circ} \mathrm{C} / \mathrm{min}$. 


\section{Synthesis of Functionalized Vinylcyclopropanes}

\section{Ethyl 1-cyano-2-vinylcyclopropane-1-carboxylate (EtVCP-CN)}

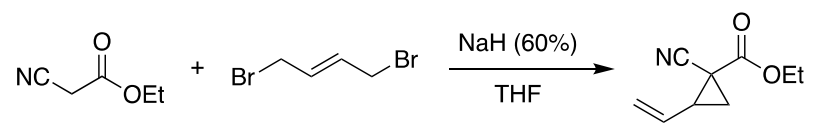

To a mixture of $\mathrm{NaH}$ (60\% pure, $4.8 \mathrm{~g}, 120.0 \mathrm{mmol}, 2.4$ equiv.) in anhydrous THF $(150 \mathrm{~mL})$ at $0{ }^{\circ} \mathrm{C}$, under $\mathrm{N}_{2}$ atmosphere, trans-1,4-dibromo-2-butene (11.0 g, $50.0 \mathrm{mmol}$, 1.0 equiv.) was added in one portion, followed by dropwise of the malonate $(5.32 \mathrm{~mL}, 50.0$ mmol, 1.0 equiv.). The mixture was then vigorously stirred at room temperature for $30 \mathrm{~min}$. The reaction was carefully quenched by slow addition of sat. $\mathrm{NH}_{4} \mathrm{Cl}$, extracted with diethyl ether three times. The combined organic layer was washed with $\mathrm{H}_{2} \mathrm{O}$ and brine, dried over anhydrous $\mathrm{Na}_{2} \mathrm{SO}_{4}$ and concentrated under vacuum. The residue was purified by vacuum distillation. Colorless oil, inseparable 7:3 mixture of diasteromers. $6.7 \mathrm{~g}, 81 \%$ yield. $\mathrm{R}_{\mathrm{f}}=$ 0.35 (30\% EtOAc in hexane). ${ }^{1} \mathbf{H}$ NMR (400 MHz, $\left.\mathrm{CDCl}_{3}\right) \delta 5.72-5.57$ (m, 1H), 5.47 $5.40(\mathrm{~m}, 1 \mathrm{H}), 5.37(\mathrm{~d}, J=10.3 \mathrm{~Hz}, 0.70 \mathrm{H}), 5.27$ (d, $J=10.4 \mathrm{~Hz}, 0.30 \mathrm{H}), 4.32-4.12(\mathrm{~m}$, 2H), $2.69-2.44(\mathrm{~m}, 1 \mathrm{H}), 2.01-1.83(\mathrm{~m}, 1.30 \mathrm{H}), 1.64(\mathrm{dd}, J=7.9,5.1 \mathrm{~Hz}, 0.70 \mathrm{H}), 1.38$ - $1.31(\mathrm{~m}, 3 \mathrm{H}) .{ }^{13} \mathbf{C}$ NMR $\left(100 \mathrm{MHz}, \mathrm{CDCl}_{3}\right) \delta 167.1,165.2,132.1,130.5,121.3,120.8$, $118.7,116.7,63.0,62.8,35.7,33.7,23.9,22.5,21.1,20.3,14.1,14.1$.

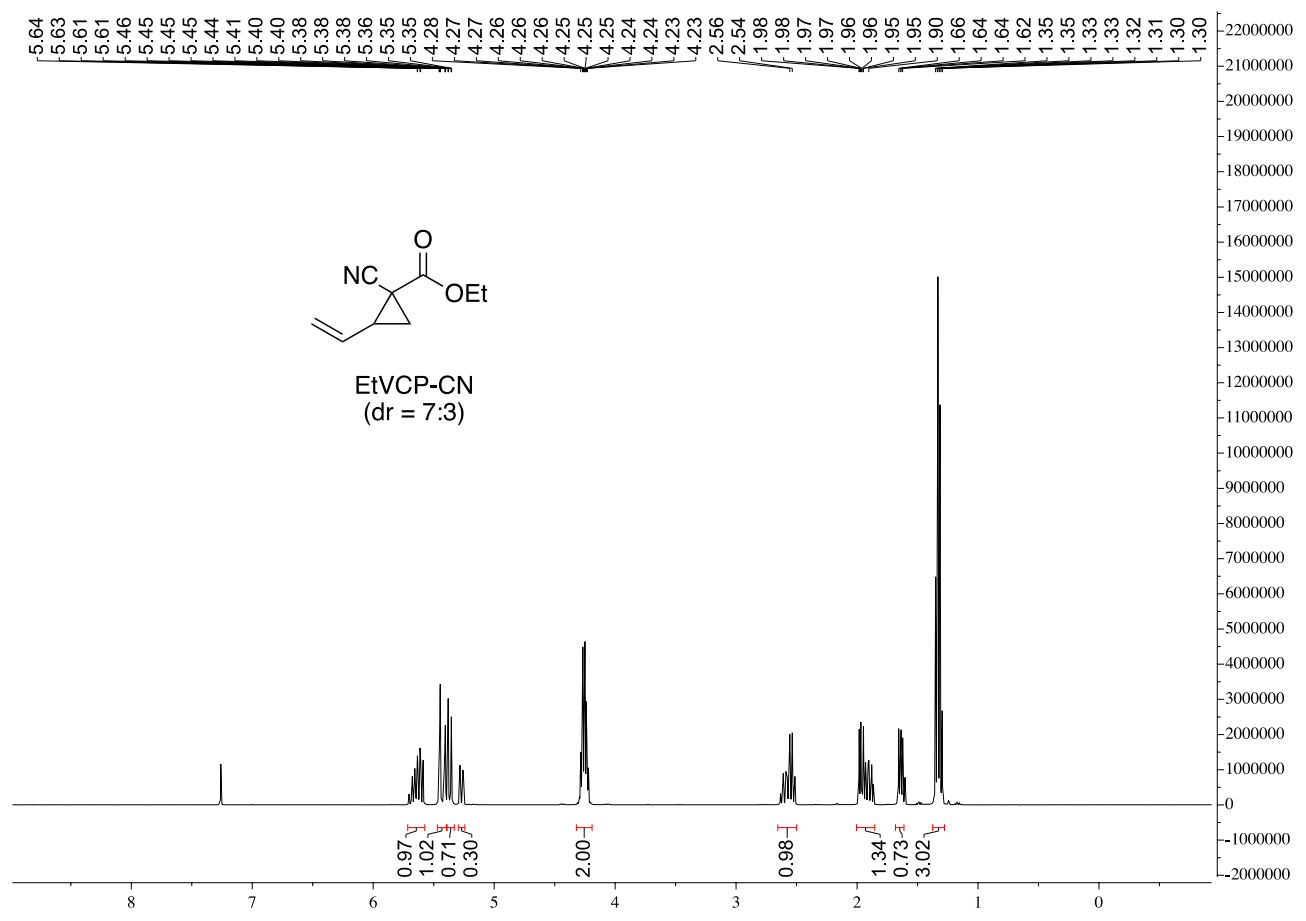

Figure S1. ${ }^{1} \mathrm{H}-\mathrm{NMR}$ spectrum of EtVCP-CN in $\mathrm{CDCl}_{3}$. 


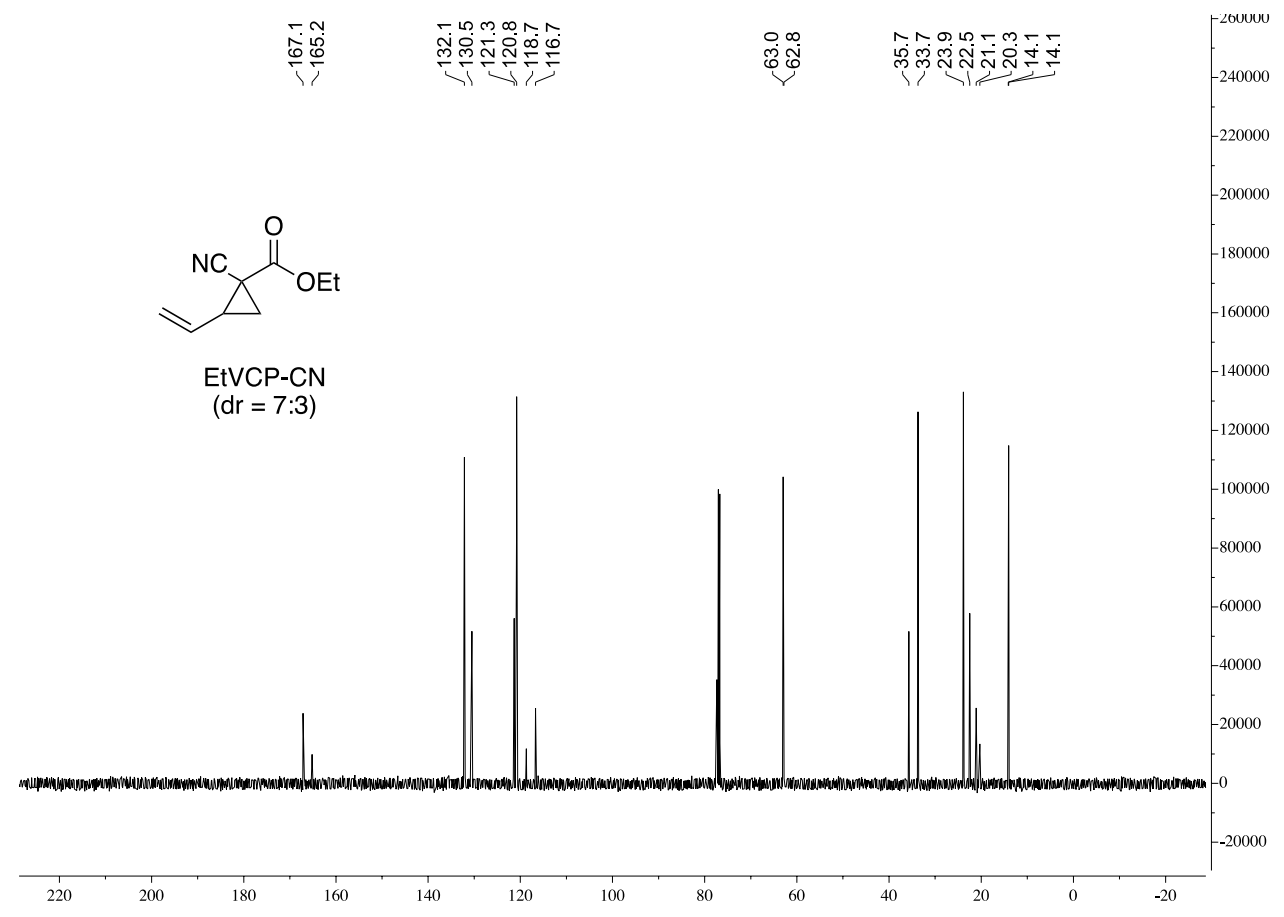

Figure S2. ${ }^{13} \mathrm{C}-\mathrm{NMR}$ spectrum of EtVCP-CN in $\mathrm{CDCl}_{3}$.

\section{Ethyl 1-(phenylcarbamoyl)-2-vinylcyclopropane-1-carboxylate (EtVCP-NHPh)}

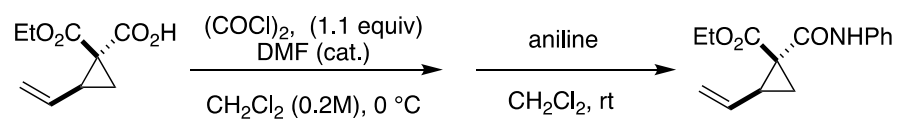

To a solution of 1-(ethoxycarbonyl)-2-vinylcyclopropane-1-carboxylic acid ${ }^{4}$ (3.68 g, $20.0 \mathrm{mmol}, 1.1$ equiv) and 3 drops of DMF in anhydrous DCM $(100 \mathrm{~mL})$ at $0{ }^{\circ} \mathrm{C}$, oxalyl chloride (1.86 mL, $22.0 \mathrm{mmol}, 1.1$ equiv) was added dropwise. The reaction was then vigorously stirred at room temperature for $3 \mathrm{~h}$. Then the mixture was cooled to $0{ }^{\circ} \mathrm{C}$, and aniline (1.82 mL, $20.0 \mathrm{mmol}, 1.0$ equiv) was added dropwise. The mixture was gradually warmed up to room temperature and stirred overnight. The white precipitate was filtered off and the filtrate was concentrated in vacuo. The residue was purified by silica gel flash chromatography using hexane/EtOAc (10:1) as the eluent to give the EtVCP-NHPh as an inseparable 10:1 mixture of diasteromers. White solid (2.78 g, 54\% yield for two steps). $\mathrm{R}_{\mathrm{f}}$ $=0.35$ (25\% EtOAc in hexane). ${ }^{1} \mathbf{H}$ NMR (400 MHz, $\left.\mathrm{CDCl}_{3}\right) \delta 10.59(\mathrm{~s}, 1 \mathrm{H}), 7.66-7.49$ (m, 2H), $7.36-7.28(\mathrm{~m}, 2 \mathrm{H}), 7.14-7.04(\mathrm{~m}, 1 \mathrm{H}), 5.71$ (ddd, $J=17.1,10.2,8.7 \mathrm{~Hz}, 1 \mathrm{H})$, $5.37(\mathrm{dd}, J=17.1,1.1 \mathrm{~Hz}, 1 \mathrm{H}), 5.21(\mathrm{dd}, J=10.1,1.2 \mathrm{~Hz}, 1 \mathrm{H}), 4.33-4.17(\mathrm{~m}, 2 \mathrm{H}), 2.64$ $(\mathrm{dd}, J=8.7,8.1 \mathrm{~Hz}, 1 \mathrm{H}), 2.20(\mathrm{dd}, J=9.2,4.4 \mathrm{~Hz}, 1 \mathrm{H}), 1.98(\mathrm{dd}, J=8.1,4.4 \mathrm{~Hz}, 1 \mathrm{H}), 1.33$ 
$(\mathrm{t}, J=7.1 \mathrm{~Hz}, 3 \mathrm{H}) .{ }^{13} \mathrm{C} \mathbf{N M R}\left(100 \mathrm{MHz}, \mathrm{CDCl}_{3}\right) \delta 171.7,166.1,138.1,133.0,128.9,124.1$, 120.0, 61.8, 38.1, 34.7, 21.7, 14.2.

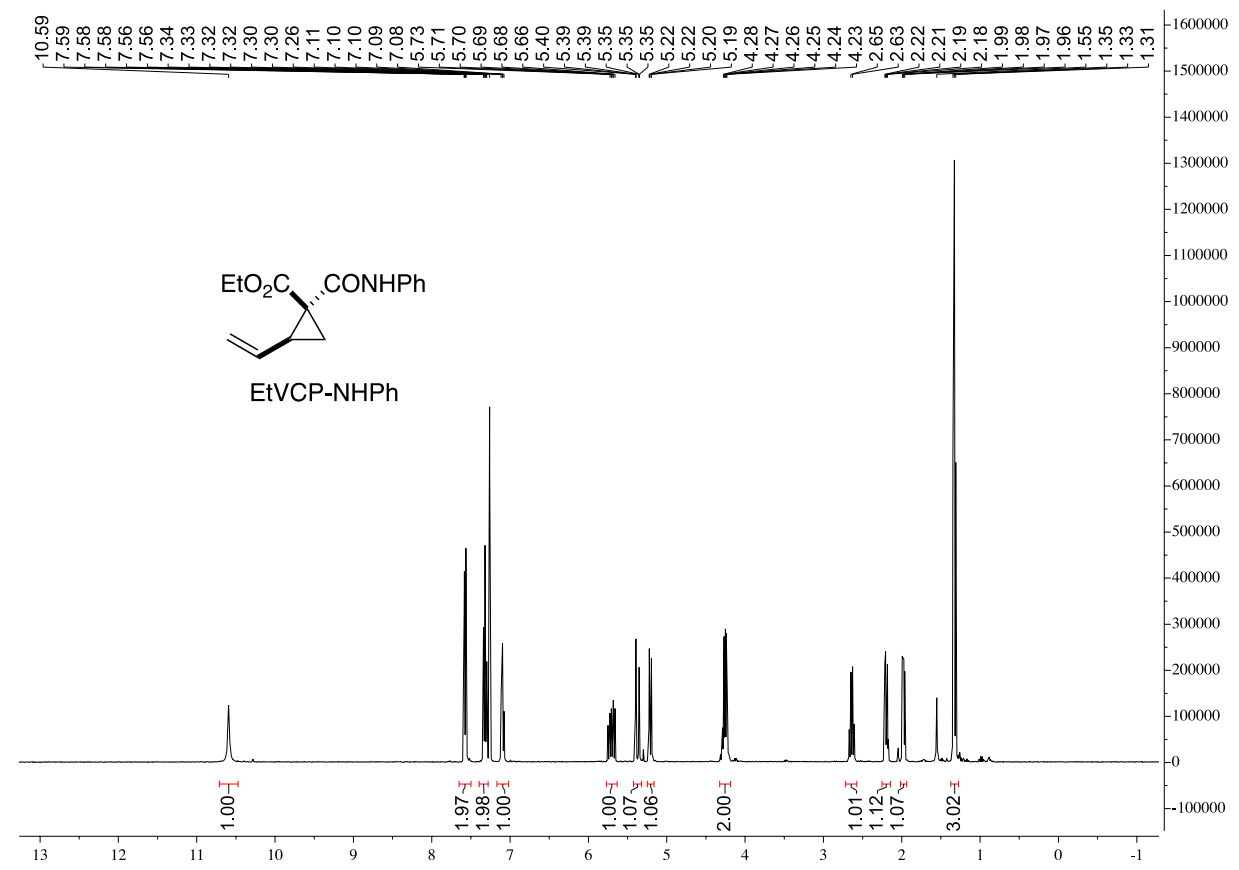

Figure S3. ${ }^{1} \mathrm{H}-\mathrm{NMR}$ spectrum of EtVCP-NHPh in $\mathrm{CDCl}_{3}$

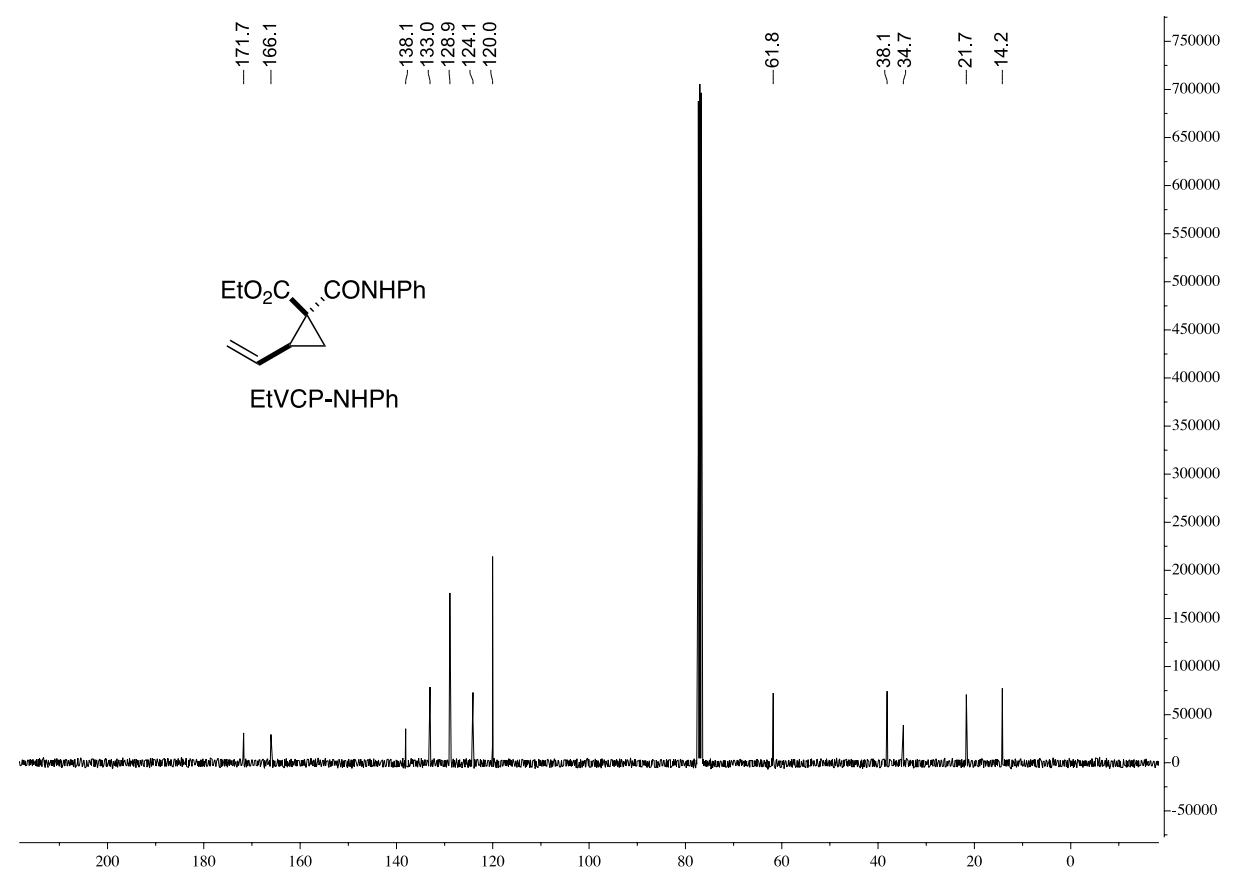

Figure S4. ${ }^{13} \mathrm{C}-\mathrm{NMR}$ spectrum of EtVCP-NHPh in $\mathrm{CDCl}_{3}$. 


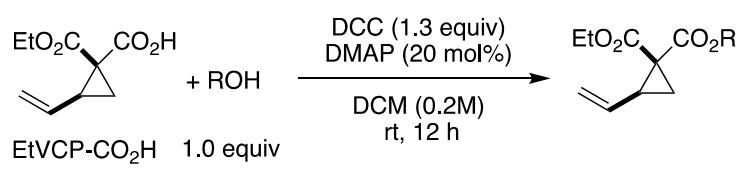

General Procedure A: To a solution of 1-(ethoxycarbonyl)-2-vinylcyclopropane-1carboxylic acid (1.1 equiv.) in anhydrous DCM $(0.2 \mathrm{M})$ at $0{ }^{\circ} \mathrm{C}$, appropriate alcohol (1.0 equiv), DMAP (0.20 equiv.), and DCC (1.3 equiv.) was sequentially added. The mixture was then vigorously stirred at room temperature for $12 \mathrm{~h}$. The white precipitate was filtered off and the filtrate was concentrated in vacuo. The residue was purified by silica gel chromatography using hexane/EtOAc as the eluent.

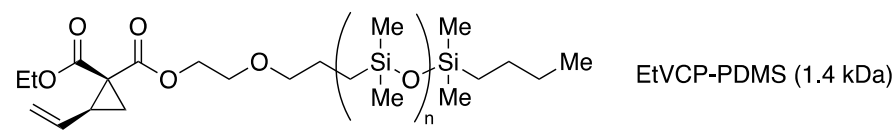

Synthesized from 1-(ethoxycarbonyl)-2-vinylcyclopropane-1-carboxylic acid and 1.0 kDa monocarbinol terminated polydimethylsiloxane (5.0 mmol scale). Colorless oil, inseparable 10:1 mixture of diasteromers. 5.23g, 88\% yield. $\mathrm{R}_{\mathrm{f}}=0.30(5 \%$ EtOAc in hexane). ${ }^{1} \mathbf{H}$ NMR $\left(400 \mathrm{MHz}, \mathrm{CDCl}_{3}\right) \delta 5.44$ (ddd, $\left.J=17.0,10.2,8.3 \mathrm{~Hz}, 1 \mathrm{H}\right), 5.29$ (dd, $J$ $=17.2,1.6 \mathrm{~Hz}, 1 \mathrm{H}), 5.13(\mathrm{dd}, J=10.1,1.7 \mathrm{~Hz}, 1 \mathrm{H}), 4.35-4.07(\mathrm{~m}, 4 \mathrm{H}), 3.62(\mathrm{t}, J=5.0$ $\mathrm{Hz}, 2 \mathrm{H}), 3.41(\mathrm{t}, J=7.0 \mathrm{~Hz}, 2 \mathrm{H}), 2.60(\mathrm{q}, J=8.3 \mathrm{~Hz}, 1 \mathrm{H}), 1.71(\mathrm{dd}, J=7.6,4.9 \mathrm{~Hz}, 1 \mathrm{H})$, $1.64-1.50(\mathrm{~m}, 3 \mathrm{H}), 1.36-1.16(\mathrm{~m}, 7 \mathrm{H}), 0.92-0.78(\mathrm{~m}, 3 \mathrm{H}), 0.59-0.46(\mathrm{~m}, 4 \mathrm{H}), 0.09-$ $0.03(\mathrm{~m}, 89 \mathrm{H}) .{ }^{13} \mathrm{C} \mathrm{NMR}\left(101 \mathrm{MHz}, \mathrm{CDCl}_{3}\right) \delta 168.6,166.2,132.0,117.5,73.1,67.2,63.7$, $60.4,34.8,30.3,25.3,24.4,22.4,19.5,16.9,13.1,13.0,12.8,0.4,0.1,0.0,-0.4,-0.9,-1.0$. $M_{\mathrm{n}}\left({ }^{1} \mathrm{H}-\mathrm{NMR}\right)=1.4 \mathrm{kDa}$.

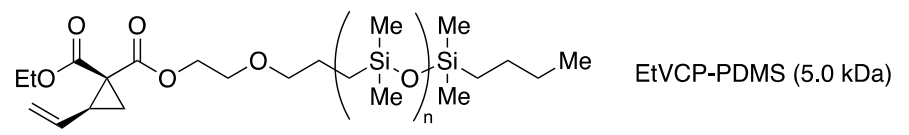

Synthesized from 1-(ethoxycarbonyl)-2-vinylcyclopropane-1-carboxylic acid and 5.0 kDa monocarbinol terminated polydimethylsiloxane (1.0 mmol scale). Colorless oil, inseparable 10:1 mixture of diasteromers. 4.75g, 91\% yield. $\mathrm{R}_{\mathrm{f}}=0.35$ (5\% EtOAc in hexane). ${ }^{1} \mathbf{H}$ NMR $\left(400 \mathrm{MHz}, \mathrm{CDCl}_{3}\right) \delta 5.44(\mathrm{ddd}, J=17.1,10.2,8.2 \mathrm{~Hz}, 1 \mathrm{H}), 5.29(\mathrm{dd}, J$ $=17.1,1.6 \mathrm{~Hz}, 1 \mathrm{H}), 5.13(\mathrm{dd}, J=10.1,1.6 \mathrm{~Hz}, 1 \mathrm{H}), 4.38-4.06(\mathrm{~m}, 4 \mathrm{H}), 3.62(\mathrm{t}, J=5.0$ $\mathrm{Hz}, 2 \mathrm{H}), 3.40(\mathrm{t}, J=7.0 \mathrm{~Hz}, 2 \mathrm{H}), 2.60(\mathrm{q}, J=8.3 \mathrm{~Hz}, 1 \mathrm{H}), 1.70(\mathrm{dd}, J=7.6,4.9 \mathrm{~Hz}, 1 \mathrm{H})$, $1.65-1.50(\mathrm{~m}, 3 \mathrm{H}), 1.35-1.15(\mathrm{~m}, 7 \mathrm{H}), 0.94-0.78(\mathrm{~m}, 3 \mathrm{H}), 0.60-0.45(\mathrm{~m}, 4 \mathrm{H}), 0.09-$ $0.03(\mathrm{~m}, 410 \mathrm{H}) .{ }^{13} \mathbf{C} \mathbf{N M R}\left(101 \mathrm{MHz}, \mathrm{CDCl}_{3}\right) \delta 168.6,166.2,132.0,117.5,73.1,67.2$, 
$63.7,60.4,34.8,30.3,25.3,24.4,22.4,19.5,16.9,13.1,13.0,12.8,0.4,0.1,0.0,-0.4,-0.9$, -1.0. $M_{\mathrm{n}}\left({ }^{1} \mathrm{H}-\mathrm{NMR}\right)=5.0 \mathrm{kDa}$.

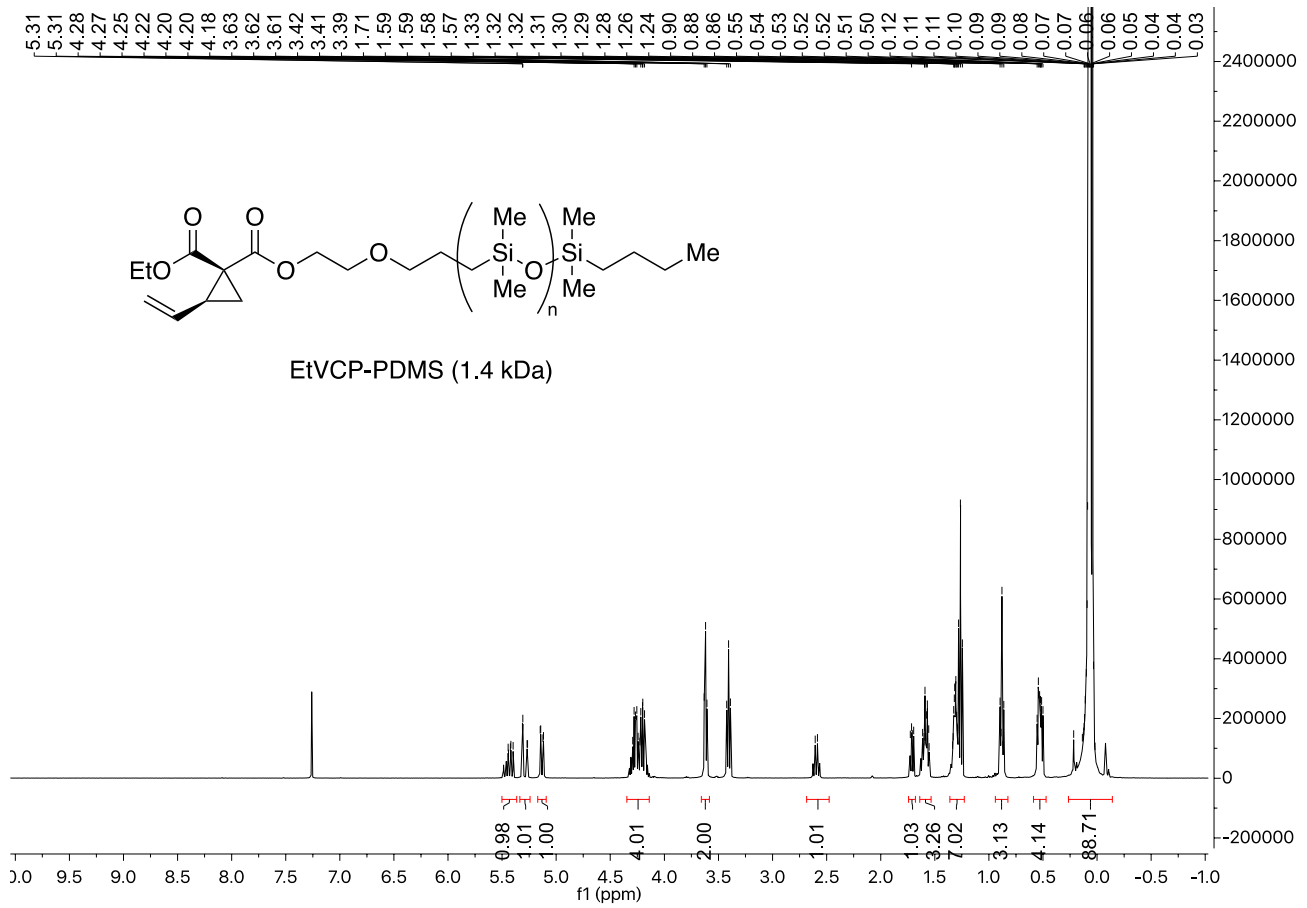

Figure S5. ${ }^{1} \mathrm{H}-\mathrm{NMR}$ spectrum of EtVCP-PDMS (1.4 kDa) in $\mathrm{CDCl}_{3}$.

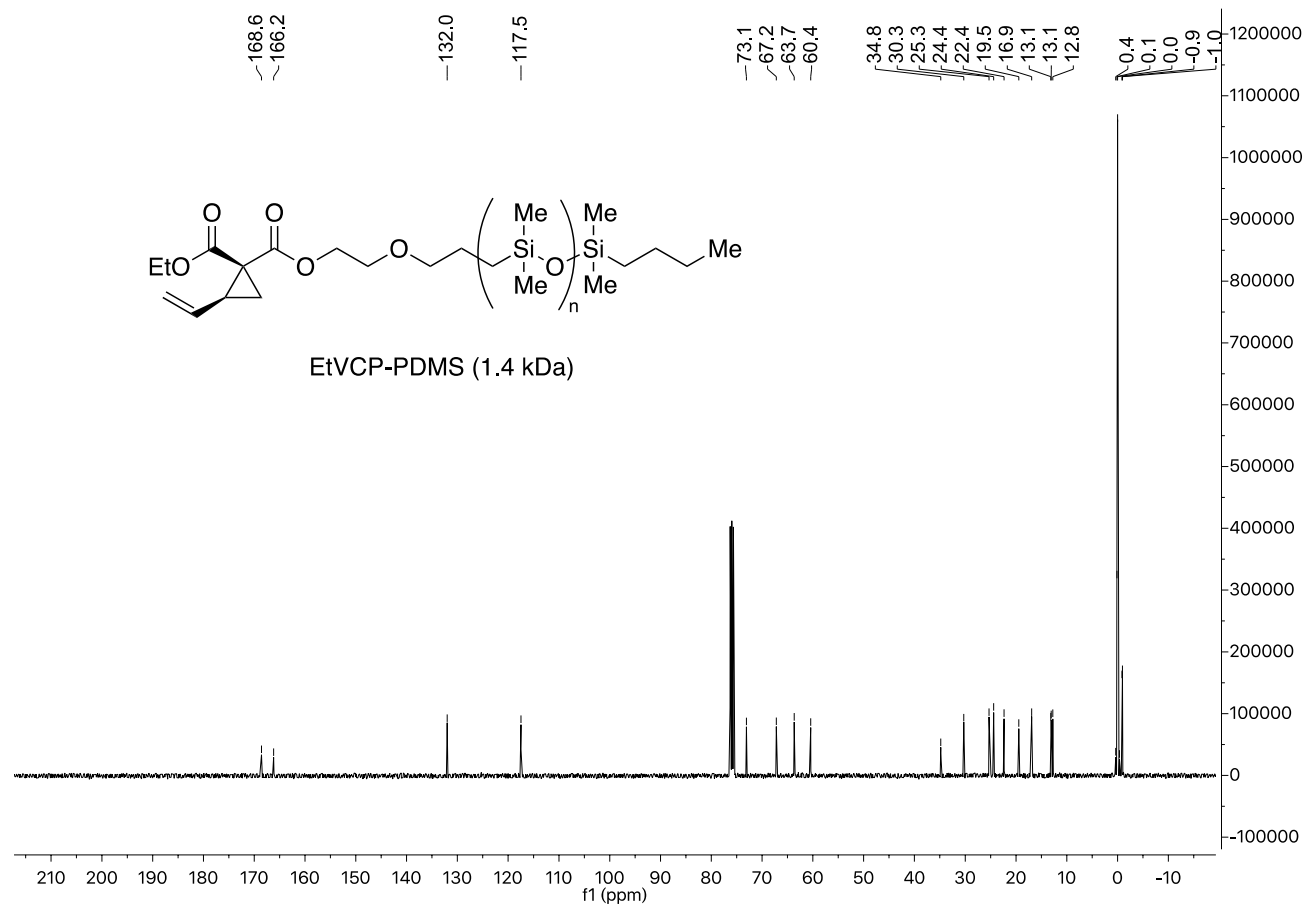

Figure S6. ${ }^{13} \mathrm{C}-\mathrm{NMR}$ spectrum of EtVCP-PDMS (1.4 kDa) in $\mathrm{CDCl}_{3}$ 


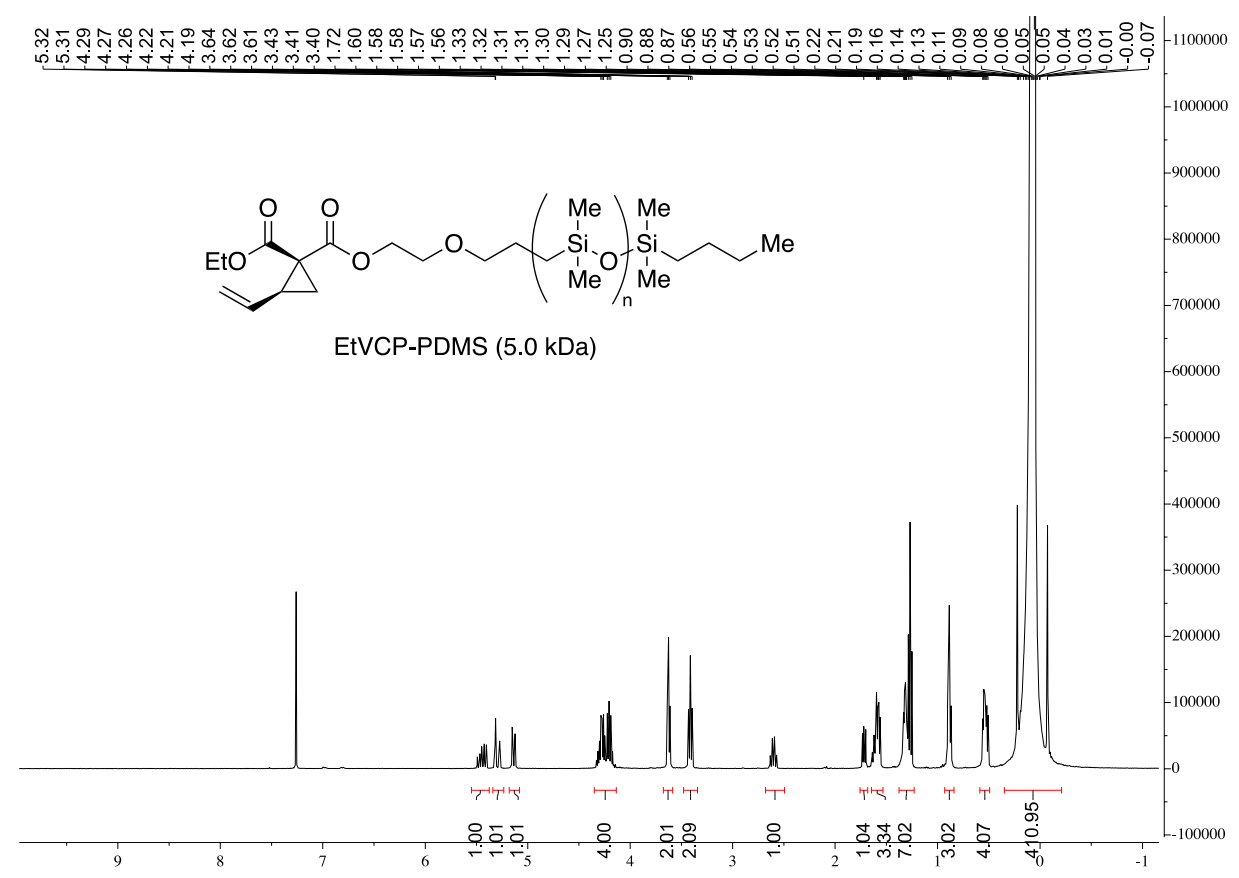

Figure S7. ${ }^{1} \mathrm{H}-\mathrm{NMR}$ spectrum of EtVCP-PDMS (5.0 kDa) in $\mathrm{CDCl}_{3}$.

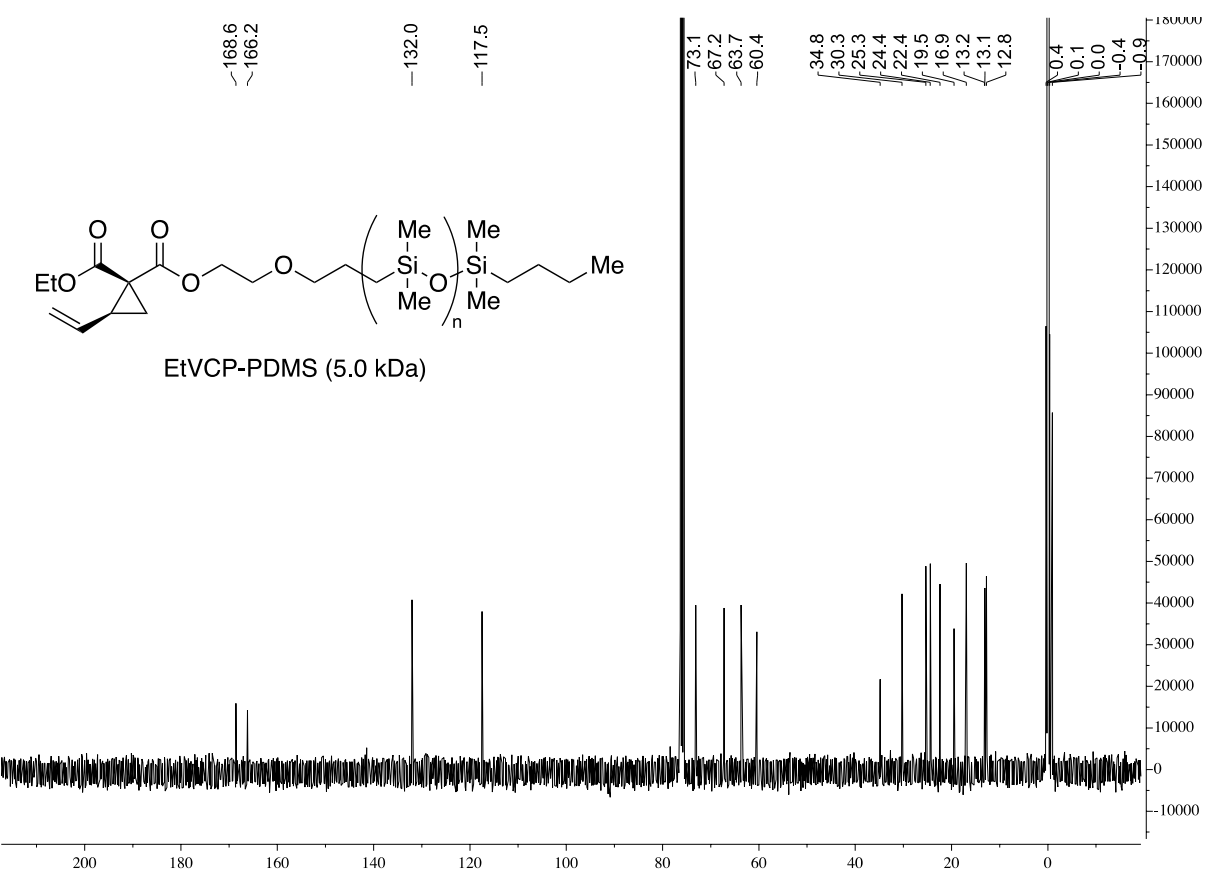

Figure S8. ${ }^{13} \mathrm{C}-\mathrm{NMR}$ spectrum of EtVCP-PDMS (5.0 kDa) in $\mathrm{CDCl}_{3}$. 


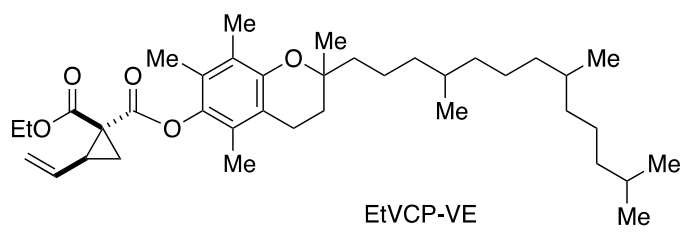

Synthesized from 1-(ethoxycarbonyl)-2-vinylcyclopropane-1-carboxylic acid and vitamin E (20.0 mmol scale). Colorless oil, inseparable 10:1 mixture of diasteromers. 11.02 g, $92 \%$ yield. $\mathrm{R}_{\mathrm{f}}=0.40$ (15\% EtOAc in hexane). ${ }^{1} \mathbf{H}$ NMR $\left(400 \mathrm{MHz}, \mathrm{CDCl}_{3}\right) \delta 5.54$ (ddd, $J=17.0,10.2,8.3 \mathrm{~Hz}, 1 \mathrm{H}), 5.37(\mathrm{dd}, J=17.0,1.5 \mathrm{~Hz}, 1 \mathrm{H}), 5.20(\mathrm{dd}, J=10.1,1.6 \mathrm{~Hz}, 1 \mathrm{H})$, $4.32-4.19(\mathrm{~m}, 2 \mathrm{H}), 2.74(\mathrm{q}, J=8.3 \mathrm{~Hz}, 1 \mathrm{H}), 2.59(\mathrm{t}, J=6.8 \mathrm{~Hz}, 2 \mathrm{H}), 2.20-1.90(\mathrm{~m}, 9 \mathrm{H})$, $1.89-1.71(\mathrm{~m}, 3 \mathrm{H}), 1.67(\mathrm{dd}, J=9.0,4.8 \mathrm{~Hz}, 1 \mathrm{H}), 1.63-1.01(\mathrm{~m}, 27 \mathrm{H}), 0.92-0.80(\mathrm{~m}$, 12H). ${ }^{13}$ C NMR $\left(101 \mathrm{MHz}, \mathrm{CDCl}_{3}\right) \delta 168.50,167.3,149.5,140.4,132.8,126.8,125.0$, 123.1, 118.8, 117.5, 75.1, 61.7, 39.4, 37.56, 37.53, 37.46, 37.42, 37.39, 37.38, 37.34, 37.29, $35.9,32.8,32.8,32.7,32.6,31.0,28.0,24.8,24.8,24.5,22.7,22.6,21.0,21.0,20.8,20.6$, $19.8,19.7,19.7,19.6,19.60,14.2,12.8,12.0,11.8$.

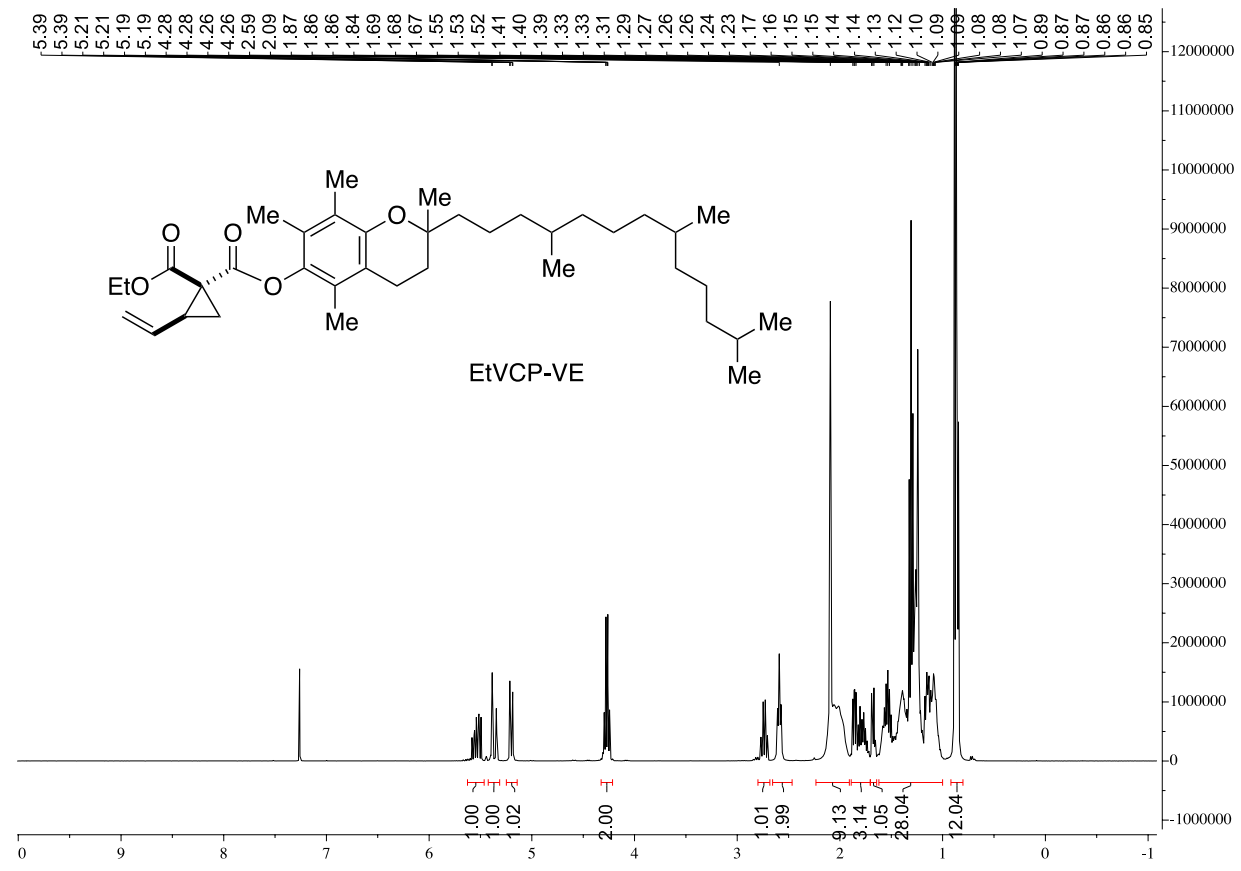

Figure S9. ${ }^{1} \mathrm{H}-\mathrm{NMR}$ spectrum of $\mathrm{EtVCP}-\mathrm{VE}$ in $\mathrm{CDCl}_{3}$. 


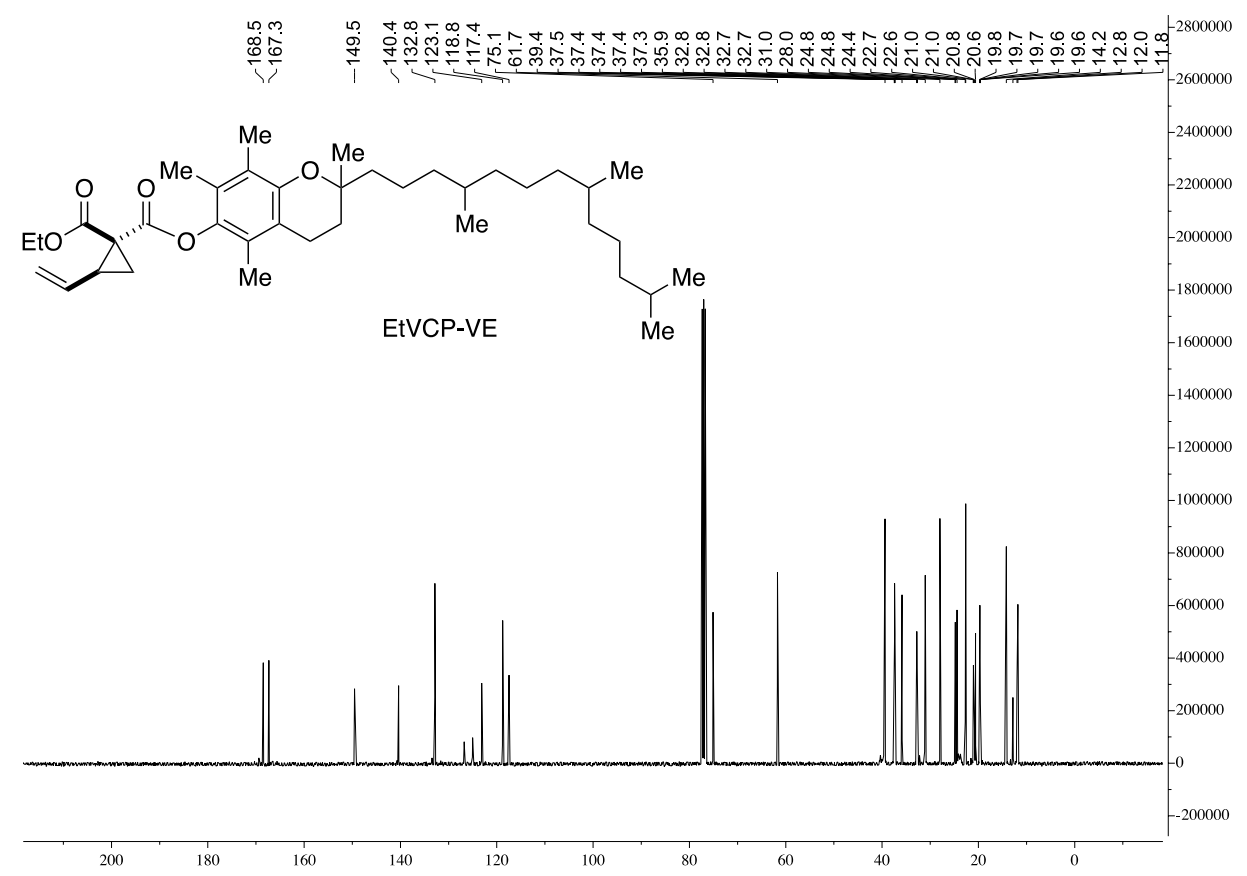

Figure S10. ${ }^{13} \mathrm{C}-\mathrm{NMR}$ spectrum of EtVCP-VE in $\mathrm{CDCl}_{3}$.

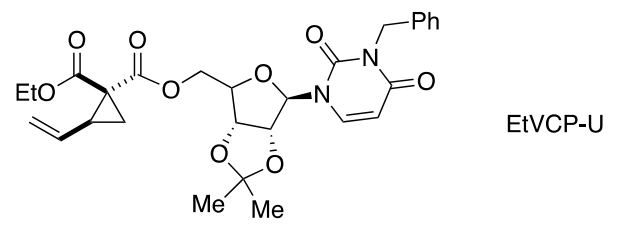

Synthesized from 1-(ethoxycarbonyl)-2-vinylcyclopropane-1-carboxylic acid and 3$N$-benzyl-2',3'-O-isopropylideneuridine ${ }^{5}$ (20.0 mmol scale). White solid, inseparable 10:1 mixture of diasteromers. $8.5 \mathrm{~g}, 79 \%$ yield. $\mathrm{R}_{\mathrm{f}}=0.30$ (70\% EtOAc in hexane). ${ }^{1} \mathbf{H}$ NMR $\left(400 \mathrm{MHz}, \mathrm{CDCl}_{3}\right) \delta 7.49-7.42(\mathrm{~m}, 2 \mathrm{H}), 7.36-7.21(\mathrm{~m}, 4 \mathrm{H}), 5.86-5.70(\mathrm{~m}, 2 \mathrm{H}), 5.51$ $-5.37(\mathrm{~m}, 1 \mathrm{H}), 5.31(\mathrm{~d}, J=17.1 \mathrm{~Hz}, 1 \mathrm{H}), 5.20-5.10(\mathrm{~m}, 2 \mathrm{H}), 5.05(\mathrm{~d}, J=13.8 \mathrm{~Hz}, 1 \mathrm{H})$, 4.89 (ddd, $J=16.6,6.5,2.4 \mathrm{~Hz}, 1 \mathrm{H}), 4.84-4.80$ (m, 1H), 4.53 (dd, $J=11.9,3.6 \mathrm{~Hz}, 0.5 \mathrm{H})$, $4.42-4.29(\mathrm{~m}, 2 \mathrm{H}), 4.27-4.09(\mathrm{~m}, 2.5 \mathrm{H}), 2.67-2.51(\mathrm{~m}, 1 \mathrm{H}), 1.76(\mathrm{ddd}, J=7.8,5.0$, $3.0 \mathrm{~Hz}, 1 \mathrm{H}), 1.63-1.58(\mathrm{~m}, 1 \mathrm{H}), 1.58-1.54(\mathrm{~m}, 3 \mathrm{H}), 1.38-1.31(\mathrm{~m}, 3 \mathrm{H}), 1.28-1.19(\mathrm{~m}$, $3 \mathrm{H}) .{ }^{13} \mathrm{C}$ NMR $\left(100 \mathrm{MHz}, \mathrm{CDCl}_{3}\right) \delta 169.32,169.28,166.9,162.5,150.8,139.5,138.8$, $136.50,136.48,132.6,132.4,129.13,129.10,128.4,127.7,119.2$, 119.2, 114.7, 114.5, $102.4,102.2,94.3,94.1,84.9,84.5,84.39,84.35,80.8,80.7,65.0,64.6,61.62,61.56,44.2$, $35.51,35.47,32.3,32.1,27.2,27.1,25.4,25.3,21.1,21.0,14.2$. 


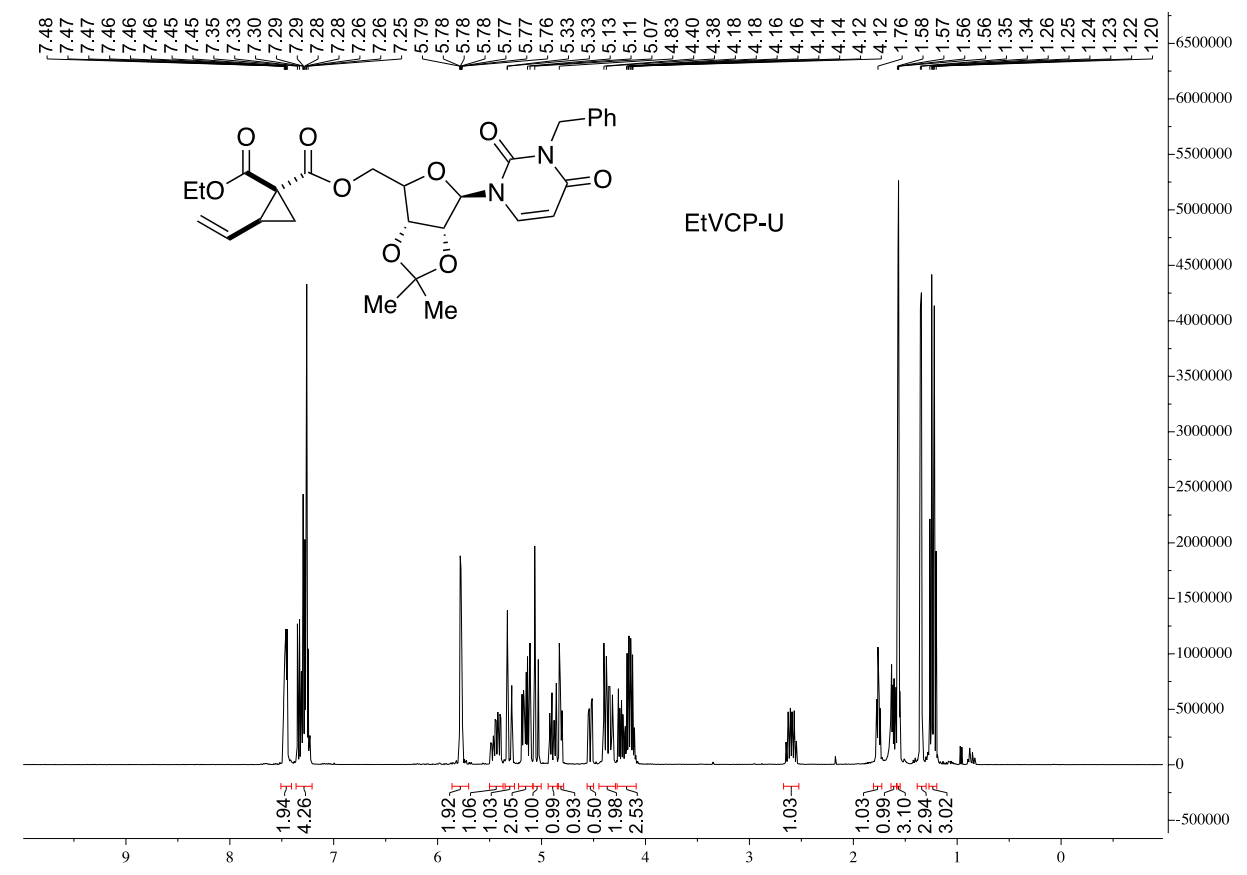

Figure S11. ${ }^{1} \mathrm{H}-\mathrm{NMR}$ spectrum of $\mathrm{EVCP}-\mathrm{U}$ in $\mathrm{CDCl}_{3}$.

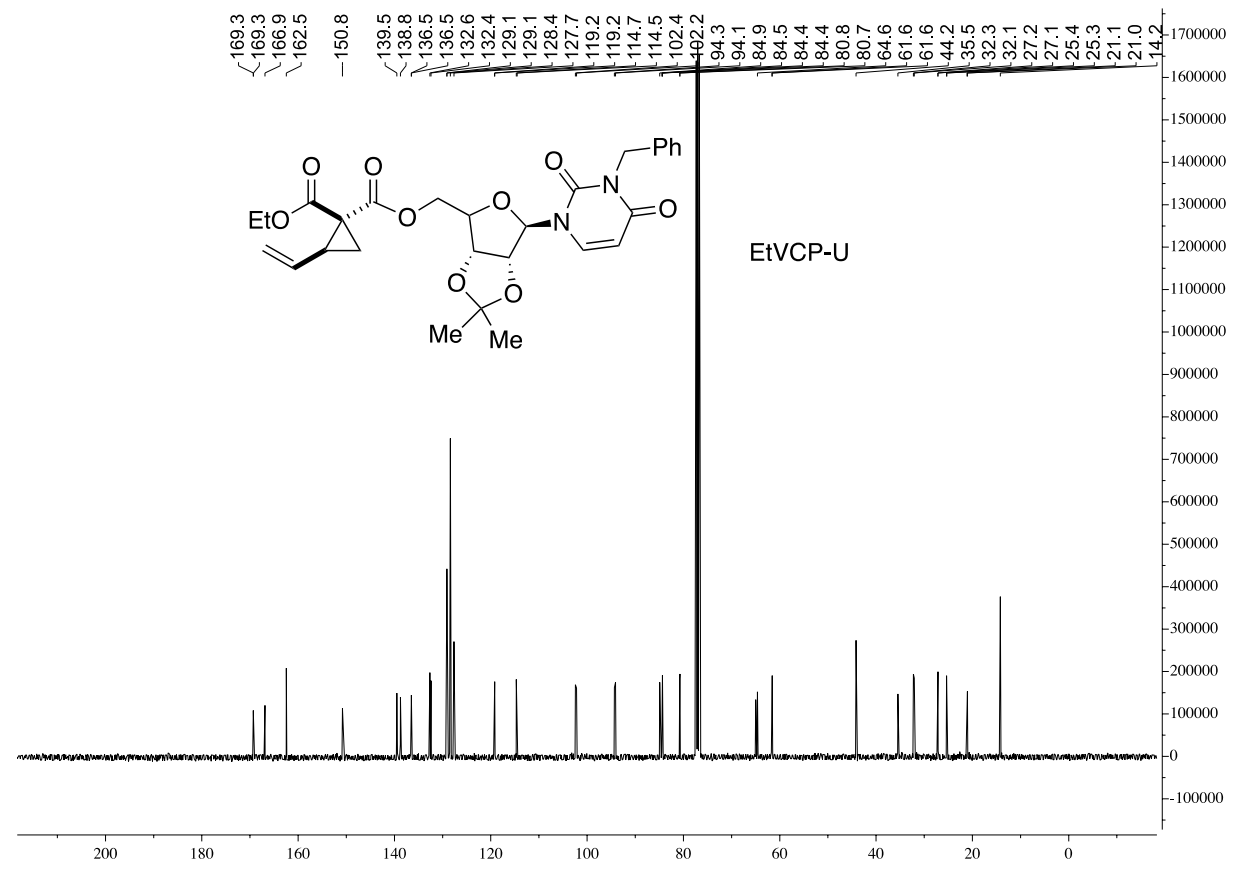

Figure S12. ${ }^{13} \mathrm{C}-\mathrm{NMR}$ spectrum of EtVCP-U in $\mathrm{CDCl}_{3}$. 


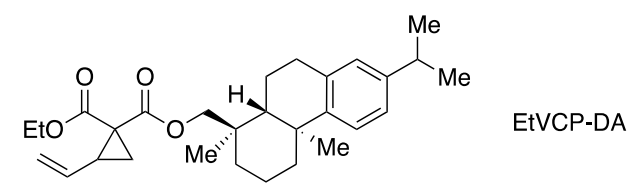

Synthesized from 1-(ethoxycarbonyl)-2-vinylcyclopropane-1-carboxylic acid and dehydroabietic methanol ${ }^{6}$ (20.0 mmol scale). White solid, inseparable 10:1 mixture of diasteromers. $6.2 \mathrm{~g}$, $68 \%$ yield. $\mathrm{R}_{\mathrm{f}}=0.35$ (20\% EtOAc in hexane). ${ }^{1} \mathbf{H}$ NMR (400 MHz, $\left.\mathrm{CDCl}_{3}\right) \delta 7.17(\mathrm{dd}, J=8.1,1.4 \mathrm{~Hz}, 1 \mathrm{H}), 6.99(\mathrm{dt}, J=8.1,2.3 \mathrm{~Hz}, 1 \mathrm{H}), 6.88(\mathrm{~d}, J=2.0 \mathrm{~Hz}$, $1 \mathrm{H}), 5.50-5.35(\mathrm{~m}, 1 \mathrm{H}), 5.32-5.22(\mathrm{~m}, 1 \mathrm{H}), 5.16-5.08(\mathrm{~m}, 1 \mathrm{H}), 4.36-3.60(\mathrm{~m}, 4 \mathrm{H})$, $2.98-2.72(\mathrm{~m}, 3 \mathrm{H}), 2.61-2.51(\mathrm{~m}, 1 \mathrm{H}), 2.36-2.24(\mathrm{~m}, 1 \mathrm{H}), 1.86-1.32(\mathrm{~m}, 10 \mathrm{H}), 1.27$ - 1.19 (m, 9H), 1.13 (t, $J=7.1 \mathrm{~Hz}, 1.7 \mathrm{H}), 1.04$ (t, $J=7.1 \mathrm{~Hz}, 1.3 \mathrm{H}), 0.96$ (d, $J=2.0 \mathrm{~Hz}$, 3H). ${ }^{13}$ C NMR (100 MHz, $\left.\mathrm{CDCl}_{3}\right) \delta 169.8,167.30,167.27,147.04,146.98,145.6,134.7$, 134.6, 133.1, 133.0, 126.8, 126.7, 124.3, 124.2, 123.9, 123.8, 118.4, 73.9, 73.8, 61.51, $61.48,44.7,44.3,38.5,38.4,37.40,37.36,37.0,36.9,35.99,35.97,35.62,35.60,33.5$, $31.19,31.17,30.2,30.0,25.3,25.2,24.04,24.01,23.98,20.5,19.1,19.0,18.51,18.47$, $17.4,17.3,14.1,14.0$.

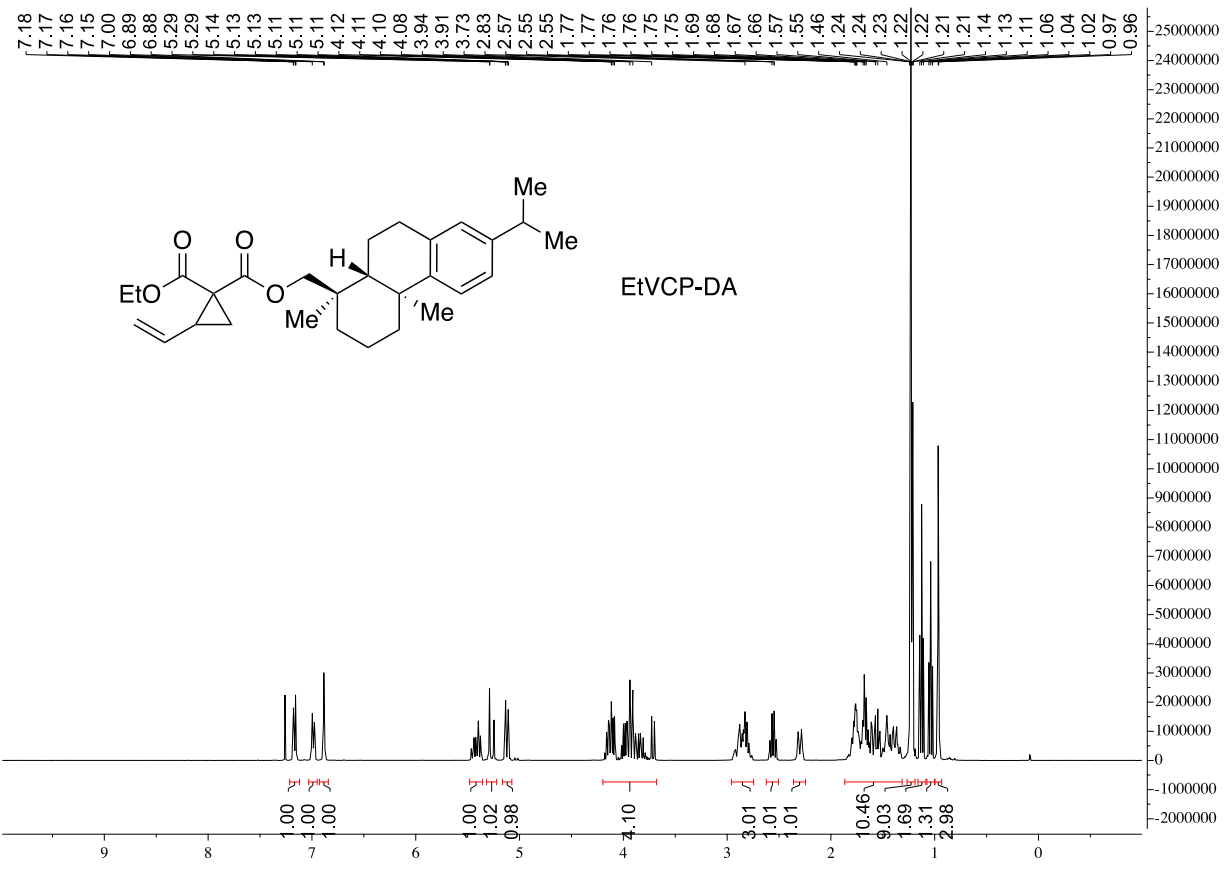

Figure S13. ${ }^{1} \mathrm{H}-\mathrm{NMR}$ spectrum of EtVCP-DA in $\mathrm{CDCl}_{3}$. 


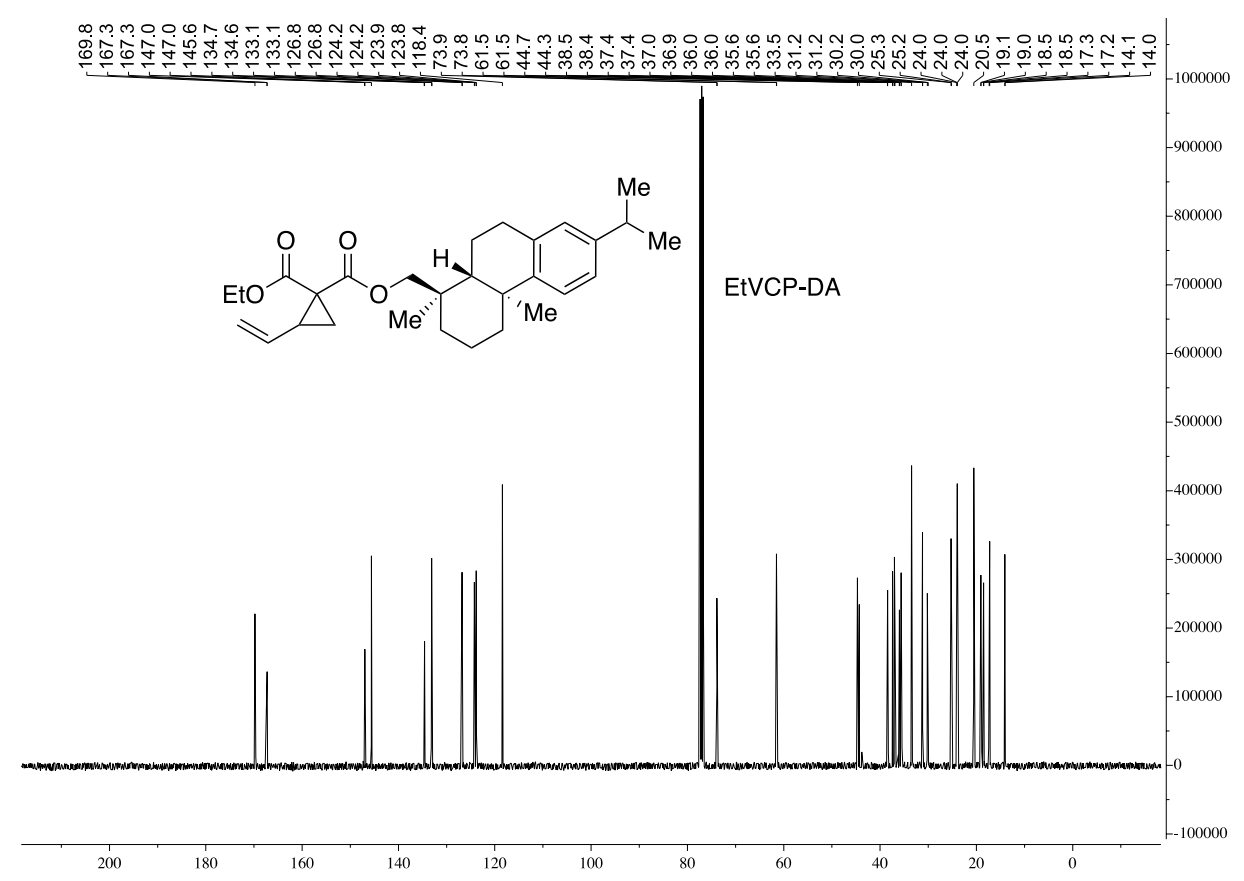

Figure S14. ${ }^{13} \mathrm{C}-\mathrm{NMR}$ spectrum of EtVCP-DA in $\mathrm{CDCl}_{3}$.

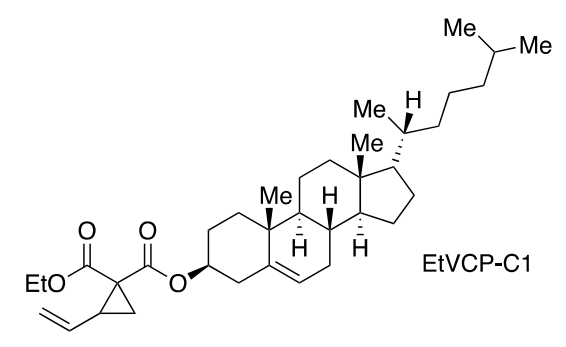

Synthesized from 1-(ethoxycarbonyl)-2-vinylcyclopropane-1-carboxylic acid and cholesterol (6.0 mmol scale). White solid, inseparable 10:1 mixture of diasteromers. 2.73 g, $82 \%$ yield. $\mathrm{R}_{\mathrm{f}}=0.40$ (5\% EtOAc in hexane). ${ }^{1} \mathbf{H}$ NMR $\left(400 \mathrm{MHz}, \mathrm{CDCl}_{3}\right) \delta 5.52-5.23$ (m, 3H), $5.12(\mathrm{dd}, J=10.1,1.7 \mathrm{~Hz}, 1 \mathrm{H}), 4.75-4.55(\mathrm{~m}, 1 \mathrm{H}), 4.34-4.09$ (m, 2H), $2.63-$ $2.47(\mathrm{~m}, 1 \mathrm{H}), 2.40-2.22(\mathrm{~m}, 2 \mathrm{H}), 2.12-1.76(\mathrm{~m}, 5 \mathrm{H}), 1.71-0.74(\mathrm{~m}, 38 \mathrm{H}), 0.67(\mathrm{~s}, 3 \mathrm{H})$. ${ }^{13}$ C NMR $\left(100 \mathrm{MHz}, \mathrm{CDCl}_{3}\right) \delta 169.0,167.5,139.4,133.2,122.8,118.3,75.3,61.4,56.7$, 56.1, 50.0, 42.3, 39.7, 39.5, 37.9, 36.9, 36.6, 36.2, 36.1, 35.8, 31.9, 31.8, 30.9, 28.2, 28.0, $27.6,24.3,23.8,22.8,22.6,21.0,20.3,19.3,18.7,14.2,11.8$. 


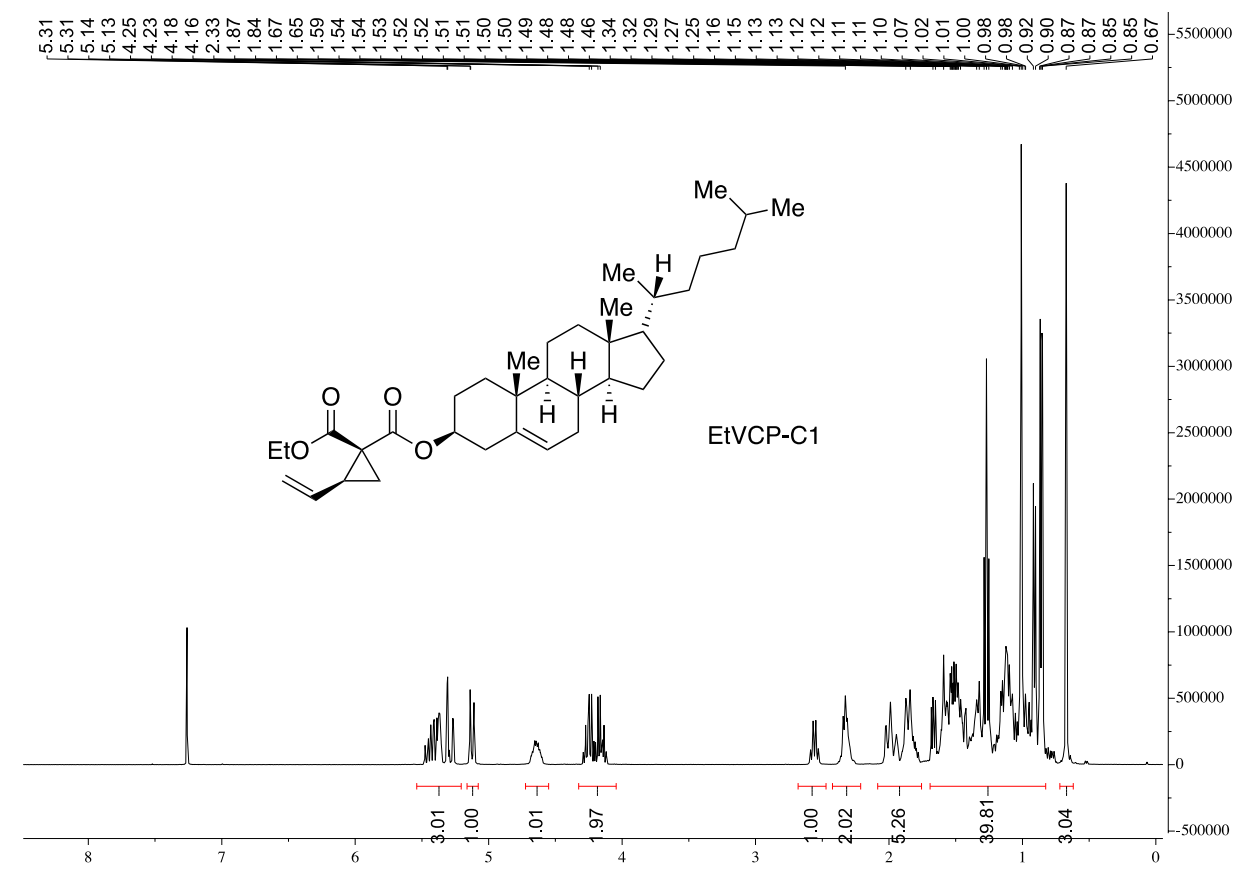

Figure S15. ${ }^{1} \mathrm{H}-\mathrm{NMR}$ spectrum of EtVCP-C1 in $\mathrm{CDCl}_{3}$.

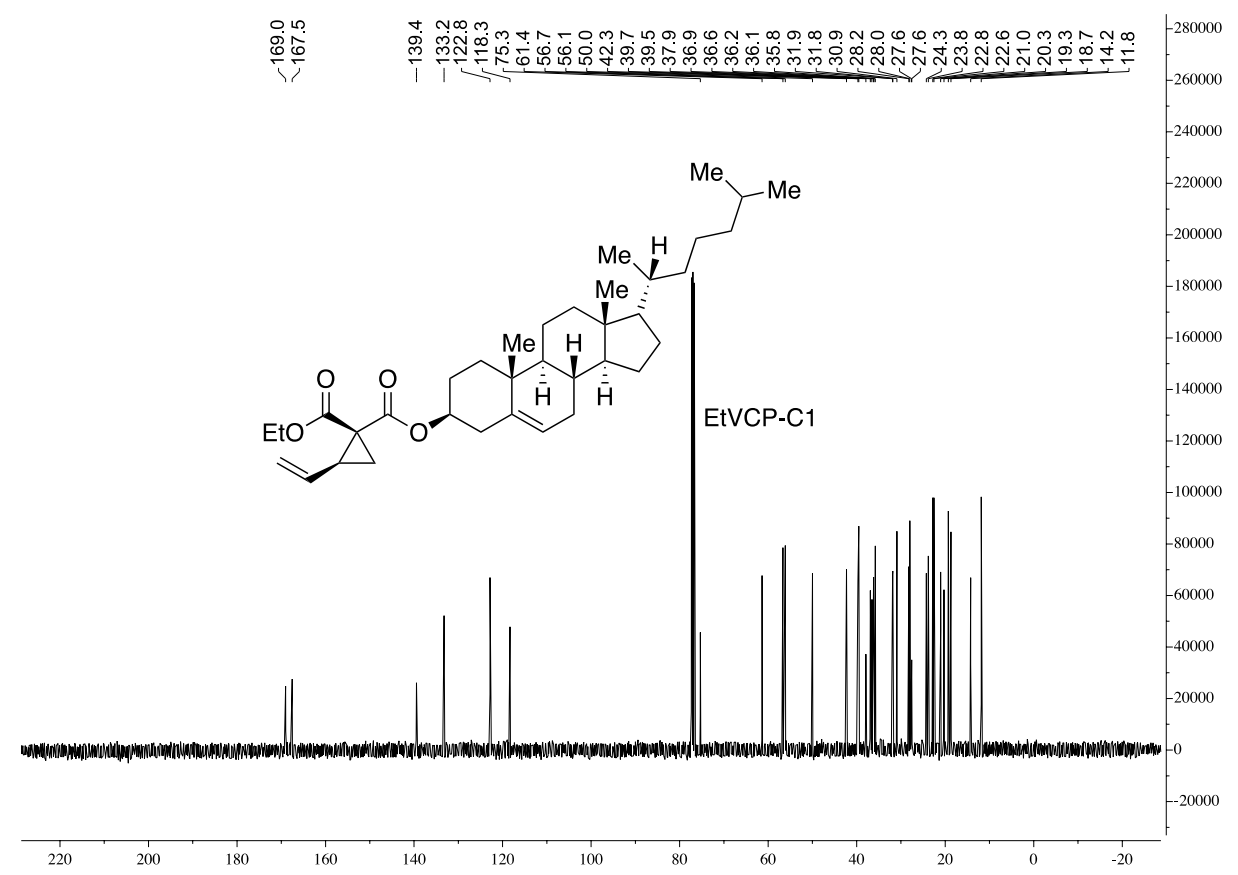

Figure S16. ${ }^{13} \mathrm{C}-\mathrm{NMR}$ spectrum of EtVCP-C1 in $\mathrm{CDCl}_{3}$. 


\section{Impact of Additives On Organocatalyzed Photoreodx Radical Ring-}

\section{Opening Polymerization of EtVCP}

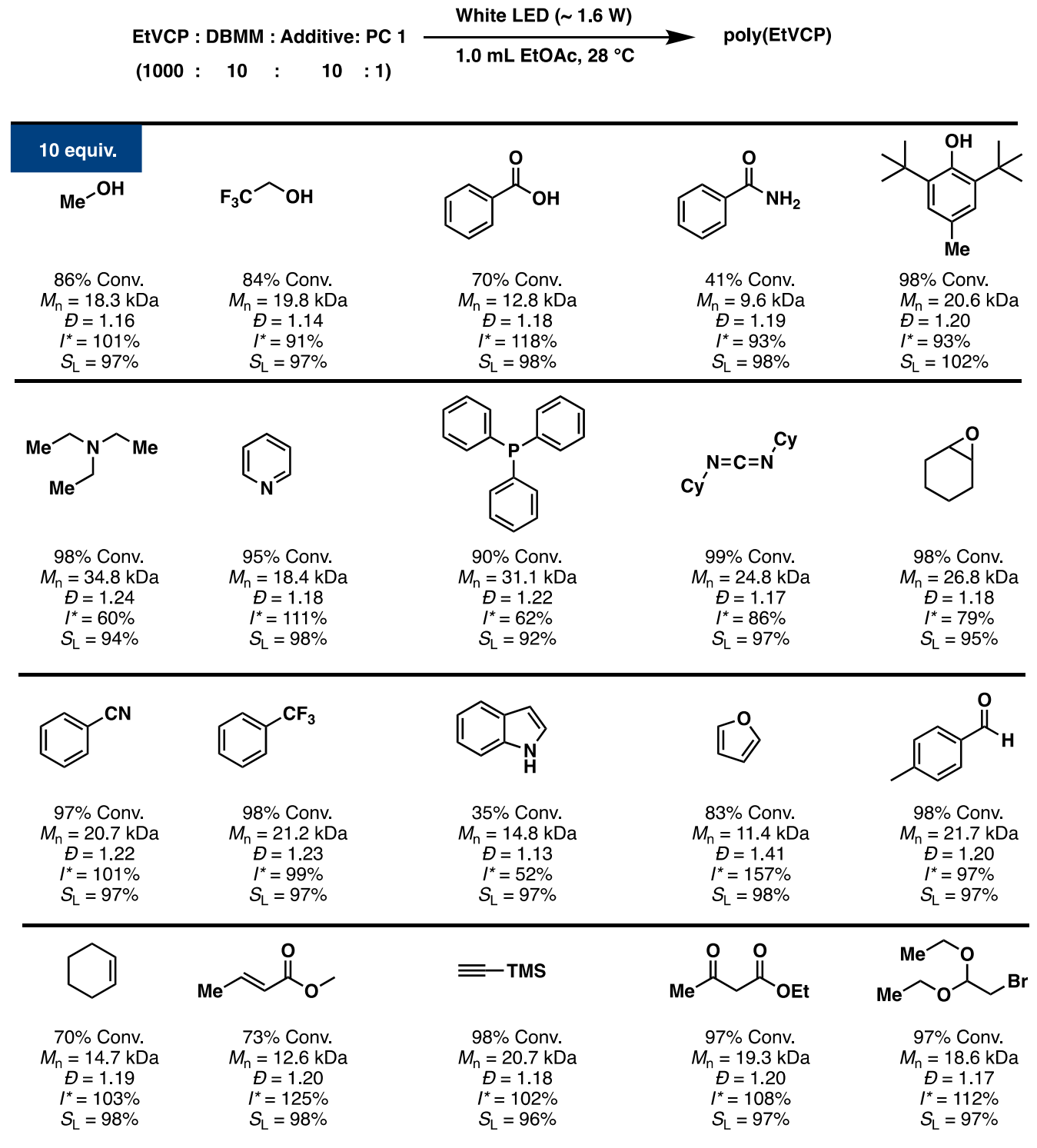

1000 equiv.
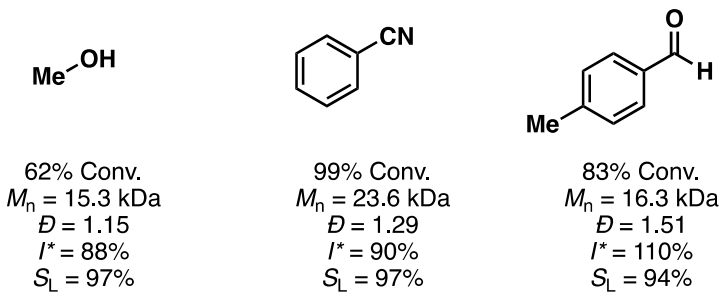
$99 \%$ Conv.
$\theta=1.29$
$I^{*}=90 \%$
$S_{\mathrm{L}}=97 \%$
$83 \%$ Conv.
$M_{\mathrm{n}}=16.3 \mathrm{kDa}$
$\theta=1.51$
$I^{*}=110 \%$
$S_{\mathrm{L}}=94 \%$

Scheme S1. Impact of additives on organocatalyzed radical ring-opening polymerization of EtVCP. 


\section{Polymerization of CNVCP, EtVCP-CN and EtVCP-NHPh}

\subsection{General Experimental Procedures}

General Procedure B: $1.0 \mathrm{~mL}$ of freshly made solution $(1.0 \mathrm{mM})$ of photoredox catalyst (PC) in anhydrous dichloromethane was added to a $20 \mathrm{~mL}$ scintillation vial equipped with a small magnetic stir bar. The solvent was removed and the vial was dried under vacuum for $12 \mathrm{~h}$, then transferred into a $\mathrm{N}_{2}$-filled glove box. To this vial, solvent, monomer (1.0 $\mathrm{mmol}$ ) and $0.10 \mathrm{M}$ of the initiator stock solution were sequentially added. The vial was then tightly capped and placed into a white-light LED beaker while stirring in the glove box. The temperature was about $28{ }^{\circ} \mathrm{C}$ with a cooling fan. For the analysis of the polymerization at a given time point, a $0.10 \mathrm{~mL}$ aliquot of the reaction mixture was taken via syringe and immediately quenched by injecting into a $1.5 \mathrm{~mL}$ vial containing $\sim 0.70 \mathrm{~mL}$ $\mathrm{CDCl}_{3}$ with $250 \mathrm{ppm}$ butylated hydroxytoluene (BHT). This aliquot was then analyzed via ${ }^{1} \mathrm{H}$ NMR for monomer conversion. The aliquot was then dried under vacuum to remove all volatiles, re-dissolved in THF and passed through a syringe filter for direct analysis by GPC. For purification, the polymerization mixture was slowly added into $50.0 \mathrm{~mL}$ of methanol while stirring at $0{ }^{\circ} \mathrm{C}$. The precipitated polymer was collected by vacuum filtration, washed with cold methanol $(5.0 \mathrm{~mL} \times 3)$ and dried overnight under vacuum at $50{ }^{\circ} \mathrm{C}$ to a constant weight.

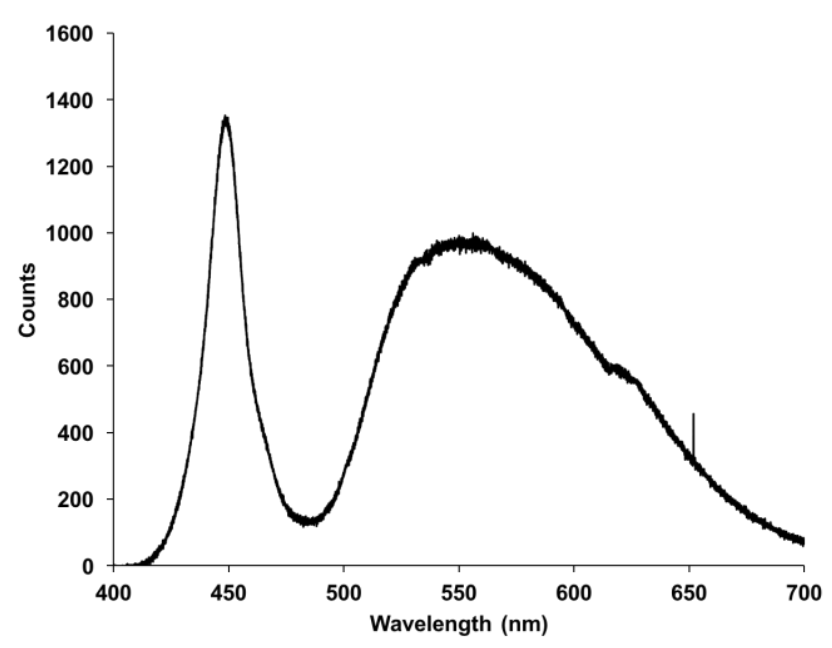

Figure S17. Emission spectra of the white LED. 
General Procedure C: $1.0 \mathrm{~mL}$ of freshly made solution $(1.0 \mathrm{mM})$ of photoredox catalyst (PC) in anhydrous dichloromethane (DCM) was added to a $25 \mathrm{~mL}$ Schlenk storage tube equipped with a small magnetic stir bar. DCM was removed under vacuum, then transferred into a $\mathrm{N}_{2}$-filled glove box. To this tube, anhydrous EtOAc, monomer (1.0 $\mathrm{mmol}$ ), and 0.10 M DBMM stock solution in anhydrous EtOAc were sequentially added. The Schlenk tube was then tightly capped, taken out of the glove box, then placed into preheat oil bath $\left(60^{\circ} \mathrm{C}\right)$ while stirring. A $34 \mathrm{~W}$ Kessil blue LED was then immediately turned on. The distance between the Schlenk tube and the blue LED was about $5 \mathrm{~cm}$. The monomer conversion and $S_{\mathrm{L}}$ were determined by crude ${ }^{1} \mathrm{H}-\mathrm{NMR}$ while $M_{\mathrm{n}}$ and $\oslash$ of the obtained polymer was determined by the GPC. Purification of the polymer followed the procedure described in General Procedure B. 


\subsection{Optimization}

Table S1. Initiator screening for polymerization of EtVCP-CN ${ }^{a}$

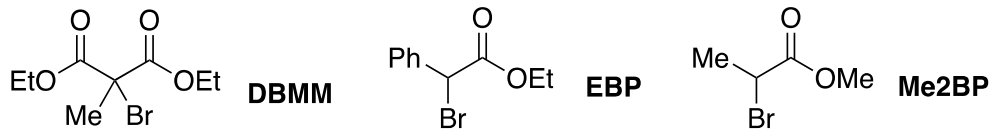

\begin{tabular}{ccccccc}
\hline Entry & Initiator & Conv. $(\boldsymbol{\%})$ & $\boldsymbol{M}_{\mathbf{n}}(\mathbf{k D a})$ & $\boldsymbol{D}\left(\boldsymbol{M}_{\mathbf{w}} / \boldsymbol{M}_{\mathbf{n}}\right)$ & $\boldsymbol{I}^{*}(\boldsymbol{\%})$ & $\boldsymbol{S}_{\mathbf{L}}(\boldsymbol{\%})$ \\
1 & DBMM & 90 & 17.6 & 1.22 & 86 & 99 \\
2 & EBP & 71 & 21.8 & 1.40 & 55 & 97 \\
3 & M2BP & 64 & 17.2 & 1.44 & 62 & 99 \\
\hline
\end{tabular}

${ }^{a}$ Polymerizations of [EtVCP-CN]/[initiator]/[1] (1000/10/1) were performed in $1.0 \mathrm{~mL}$ of anhydrous EtOAc for $12 \mathrm{~h}$, with white LEDs irradiation at $28^{\circ} \mathrm{C}$.

Table S2. Solvent screening for polymerization of EtVCP-CN ${ }^{a}$

\begin{tabular}{ccccccc}
\hline Entry & Solvent & Conv. $(\boldsymbol{\%})$ & $\boldsymbol{M}_{\mathbf{n}}(\mathbf{k D a})$ & $\boldsymbol{D}\left(\boldsymbol{M}_{\mathbf{w}} / \boldsymbol{M}_{\mathbf{n}}\right)$ & $\boldsymbol{I}^{*}(\boldsymbol{\%})$ & $\boldsymbol{S}_{\mathbf{L}}(\boldsymbol{\%})$ \\
1 & DMAc & 76 & 19.3 & 1.31 & 66 & 99 \\
2 & EtOAc & 90 & 17.6 & 1.22 & 86 & 99 \\
3 & PhCl & 85 & 17.2 & 1.47 & 83 & 98 \\
4 & DCE & 51 & 14.9 & 1.64 & 58 & 99 \\
\hline
\end{tabular}

${ }^{a}$ Polymerizations of [EtVCP-CN]/[DBMM]/[1] (1000/10/1) were performed in $1.0 \mathrm{~mL}$ of anhydrous solvent for $12 \mathrm{~h}$, with white LEDs irradiation at $28^{\circ} \mathrm{C}$. DCE $=1,2$-dichloroethane

Table S3. PC screening for polymerization of ${\mathrm{EtVCP}-\mathrm{CN}^{a}}^{a}$

\begin{tabular}{ccccccc}
\hline Entry & PC & Conv. $(\boldsymbol{\%})$ & $\boldsymbol{M}_{\mathbf{n}}(\mathbf{k D a})$ & $\boldsymbol{D}\left(\boldsymbol{M}_{\mathbf{w}} / \boldsymbol{M}_{\mathbf{n}}\right)$ & $\boldsymbol{I}^{*}(\boldsymbol{\%})$ & $\boldsymbol{S}_{\mathbf{L}}(\boldsymbol{\%})$ \\
1 & $\mathbf{1}$ & 90 & 17.6 & 1.22 & 86 & 99 \\
2 & $\mathbf{2}$ & 53 & 10.3 & 1.18 & 87 & 99 \\
3 & $\mathbf{3}$ & 66 & 19.9 & 1.33 & 56 & 97 \\
\hline
\end{tabular}

${ }^{a}$ Polymerizations of $[$ EtVCP-CN]/[DBMM]/[PC] (1000/10/1) were performed in $1.0 \mathrm{~mL}$ of anhydrous EtOAc for $12 \mathrm{~h}$, with white LEDs irradiation at $28^{\circ} \mathrm{C}$.

Table S4. Control experiments ${ }^{a}$

\begin{tabular}{ccccccccc}
\hline Entry & Light & PC 1 & DBMM & $\begin{array}{c}\text { Conv. } \\
(\%)\end{array}$ & $\begin{array}{c}M_{\mathrm{n}(\mathrm{GPC})} \\
(\mathrm{kDa})\end{array}$ & $\begin{array}{c}Ð \\
\left(M_{\mathrm{w}} / M_{\mathrm{n}}\right)\end{array}$ & $I^{*}(\%)$ & $S_{\mathrm{L}}(\%)$ \\
1 & - & - & - & 0 & - & - & - & - \\
2 & - & $\sqrt{ }$ & - & 0 & - & - & - & - \\
3 & - & - & $\sqrt{ }$ & 0 & - & - & - & - \\
4 & - & $\sqrt{ }$ & $\sqrt{ }$ & 0 & - & - & - & - \\
5 & $\sqrt{ }$ & - & - & 0 & - & - & - & - \\
6 & $\sqrt{ }$ & $\sqrt{ }$ & - & 0 & - & - & - & - \\
7 & $\sqrt{ }$ & - & $\sqrt{ }$ & 0 & - & - & - & - \\
\hline
\end{tabular}

${ }^{a}$ Polymerizations of [EtVCP-CN]/[DBMM]/[1] (1000/10/1) were performed in $1.0 \mathrm{~mL}$ of anhydrous EtOAc for $12 \mathrm{~h}$, with white LEDs irradiation at $28^{\circ} \mathrm{C}$. 


\section{3 ${ }^{1}$ H-NMR Spectrum of Poly(EtVCP-CN) and Poly(EtVCP-NHPh)}

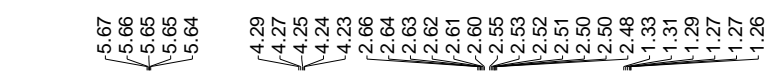

$\left(17.6 \mathrm{kDa}, \Theta=1.22, S_{\mathrm{L}}=99 \%\right)$

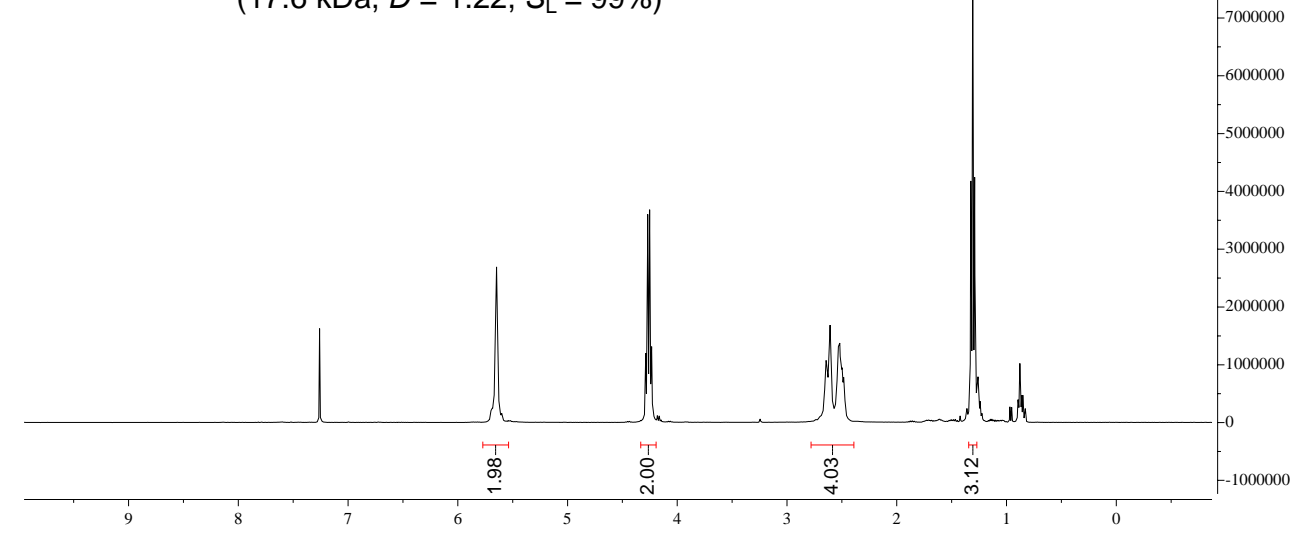

Figure S18. ${ }^{1} \mathrm{H}-\mathrm{NMR}$ spectra of poly(EtVCP-CN).

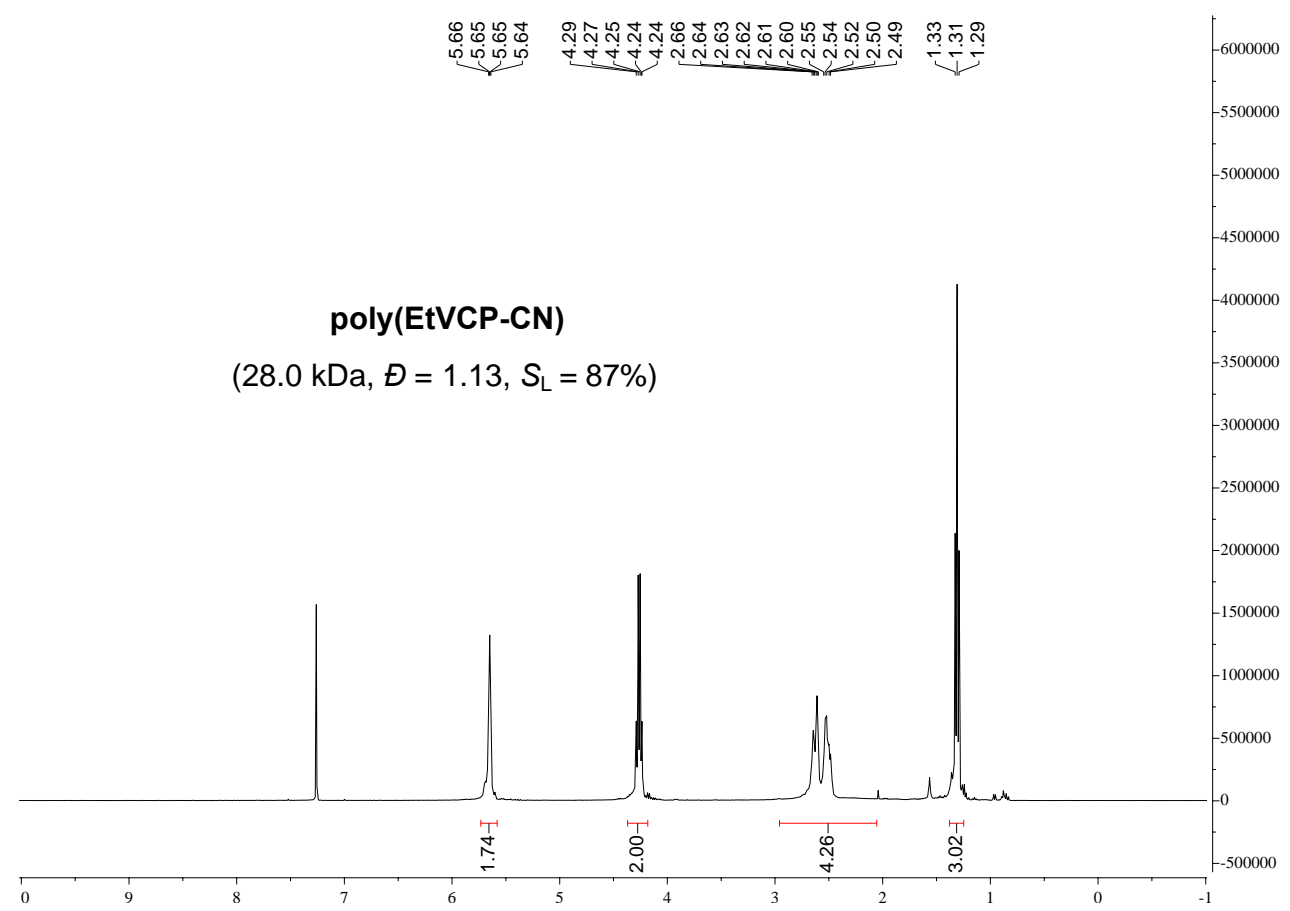

Figure S19. ${ }^{1} \mathrm{H}-\mathrm{NMR}$ spectra of poly(EtVCP-CN). 


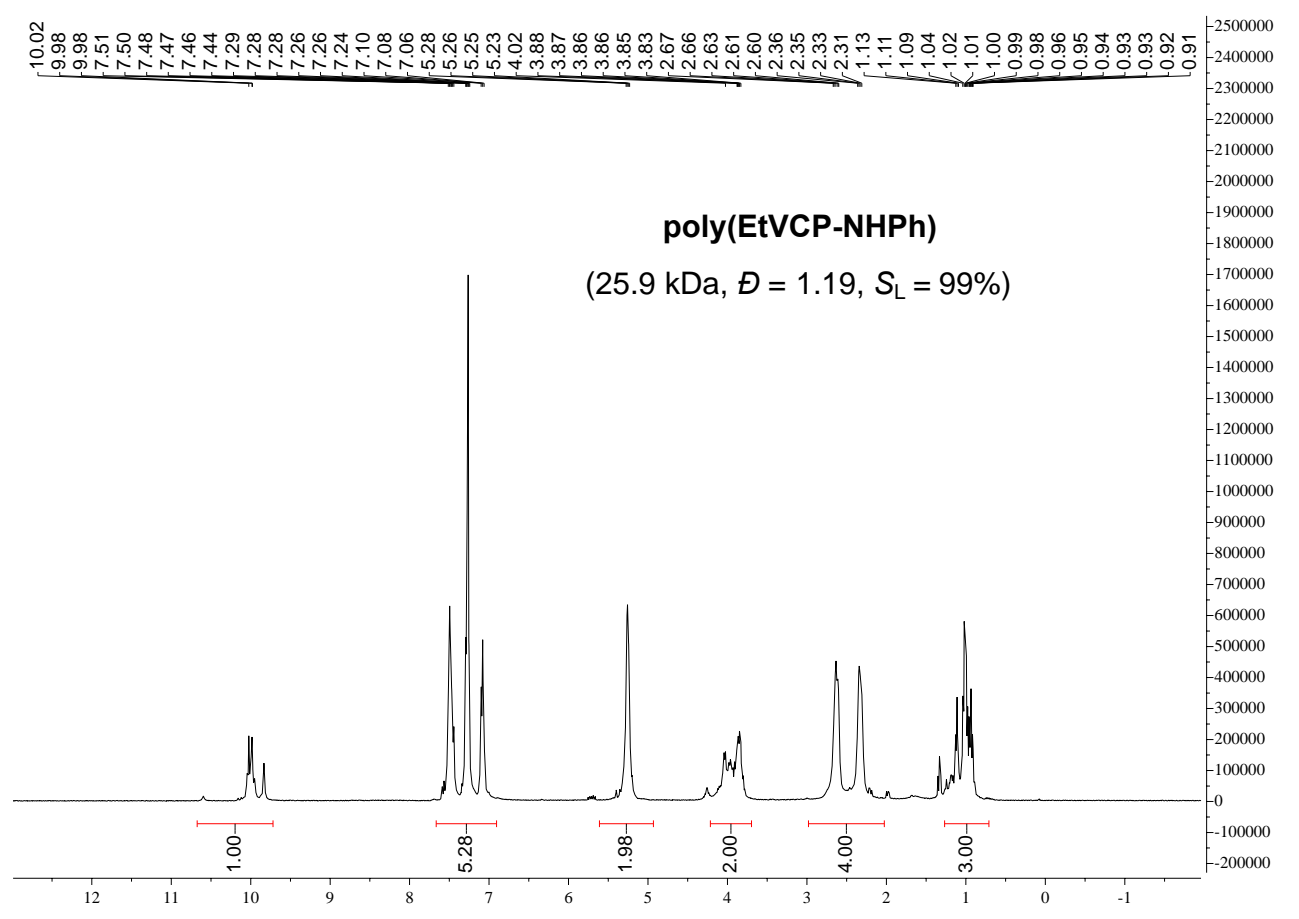

Figure S20. ${ }^{1} \mathrm{H}-\mathrm{NMR}$ spectra of poly(EtVCP-NHPh).

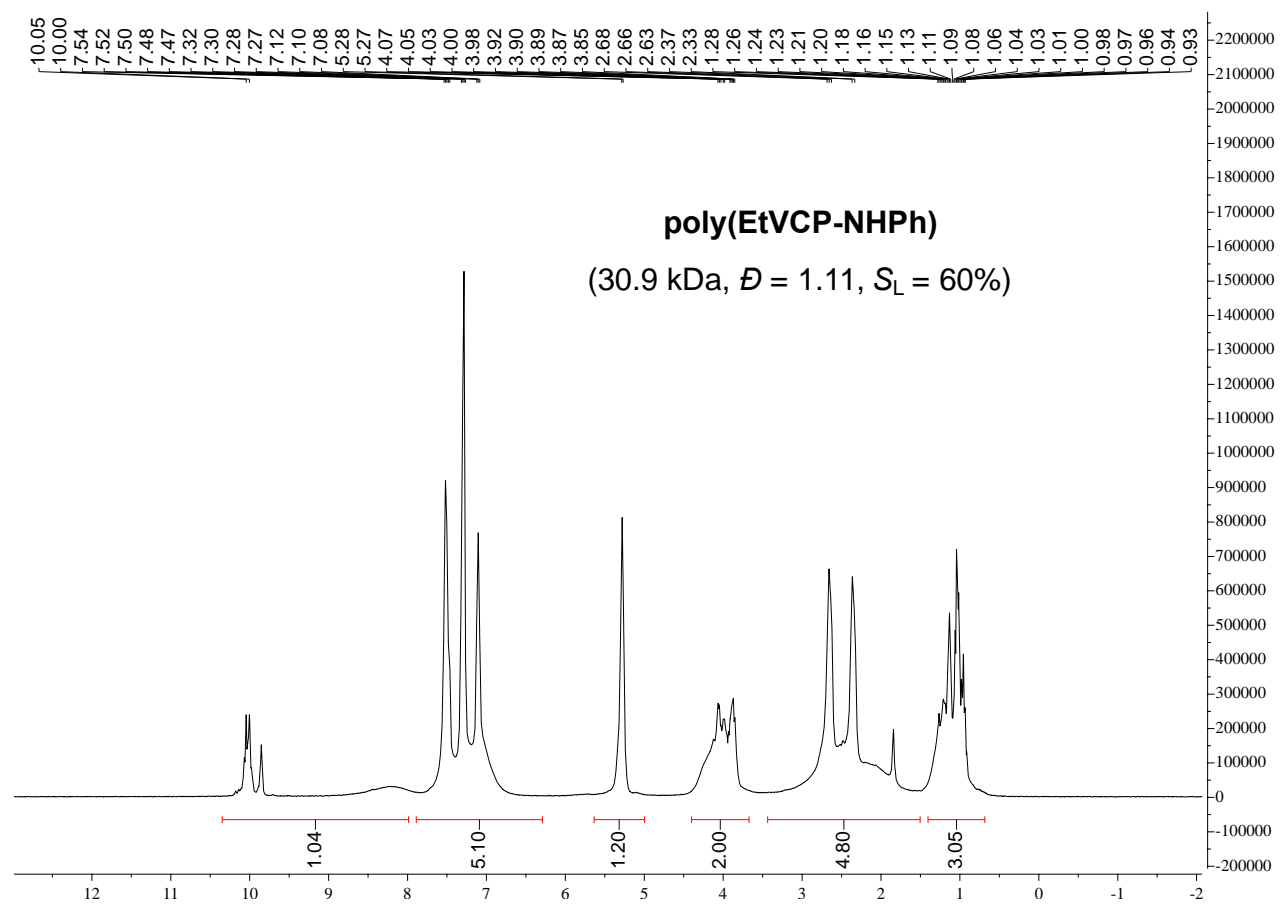

Figure S21. ${ }^{1} \mathrm{H}-\mathrm{NMR}$ spectra of poly(EtVCP-NHPh). 


\subsection{GPC Traces of Poly(EtVCP-CN) and Poly(EtVCP-NHPh)}

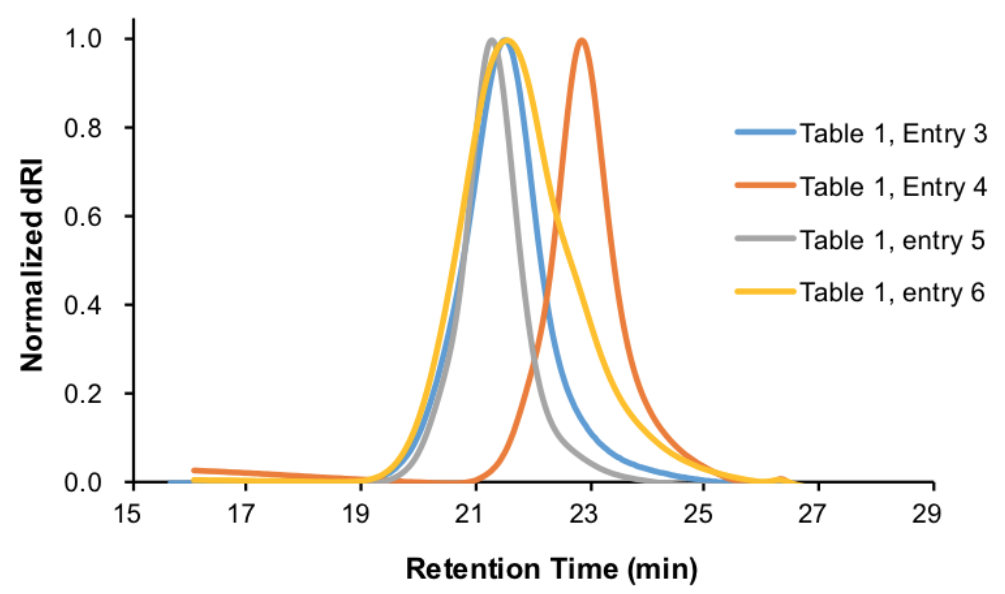

Figure S22. Overlay of GPC traces for poly(EtVCP-CN) in Table 1.

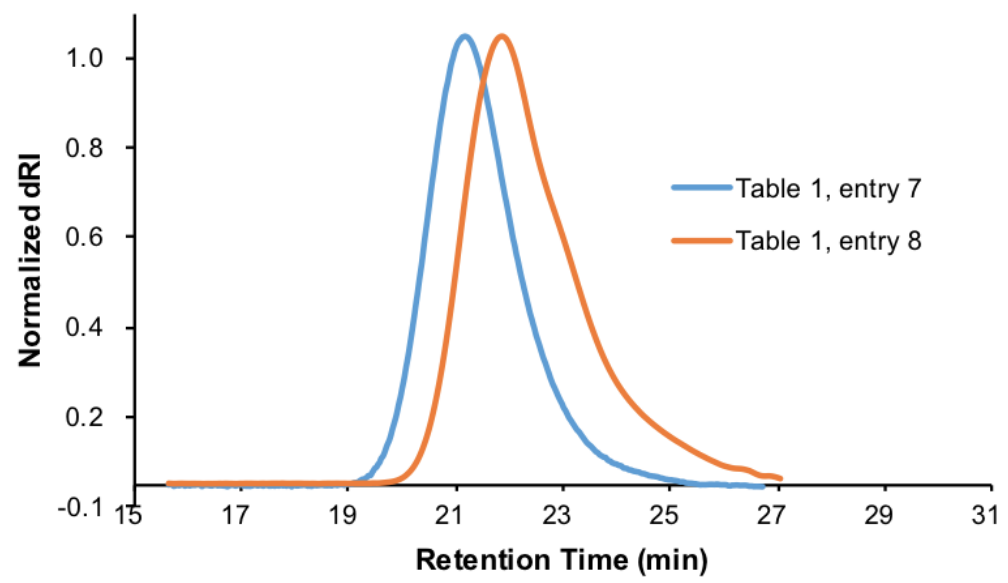

Figure S23. Overlay of GPC traces for poly(EtVCP-NHPh) in Table 1. 


\subsection{DCS and TGA Analysis of Poly(EtVCP-CN) and Poly(EtVCP-NHPh)}

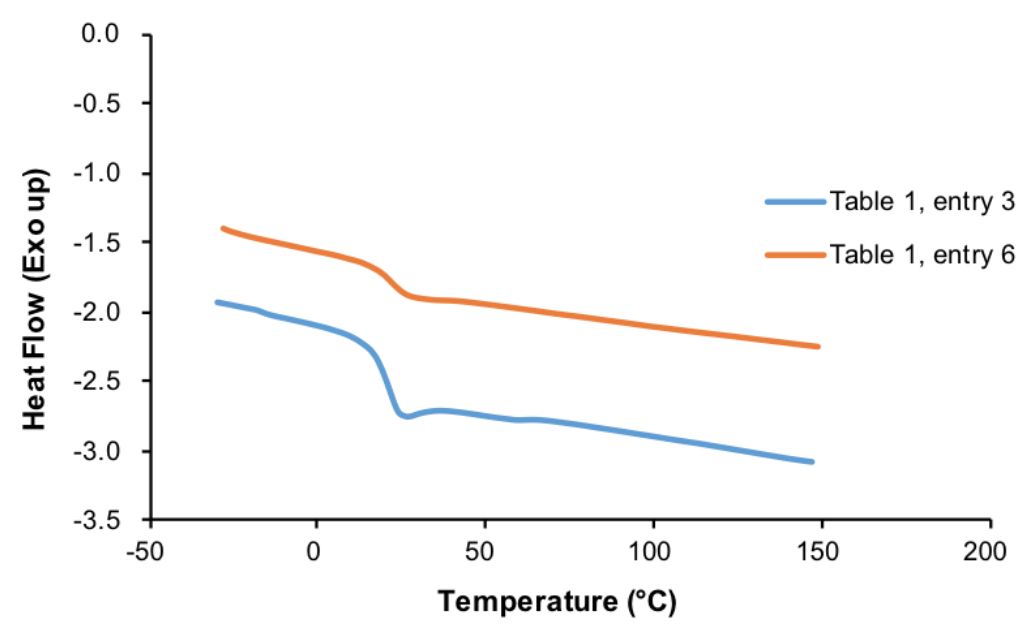

Figure S24. Overlay of DSC curves for poly(EtVCP-CN) in Table 1, entry $3\left(23^{\circ} \mathrm{C}\right)$ and entry $6\left(21^{\circ} \mathrm{C}\right)$.

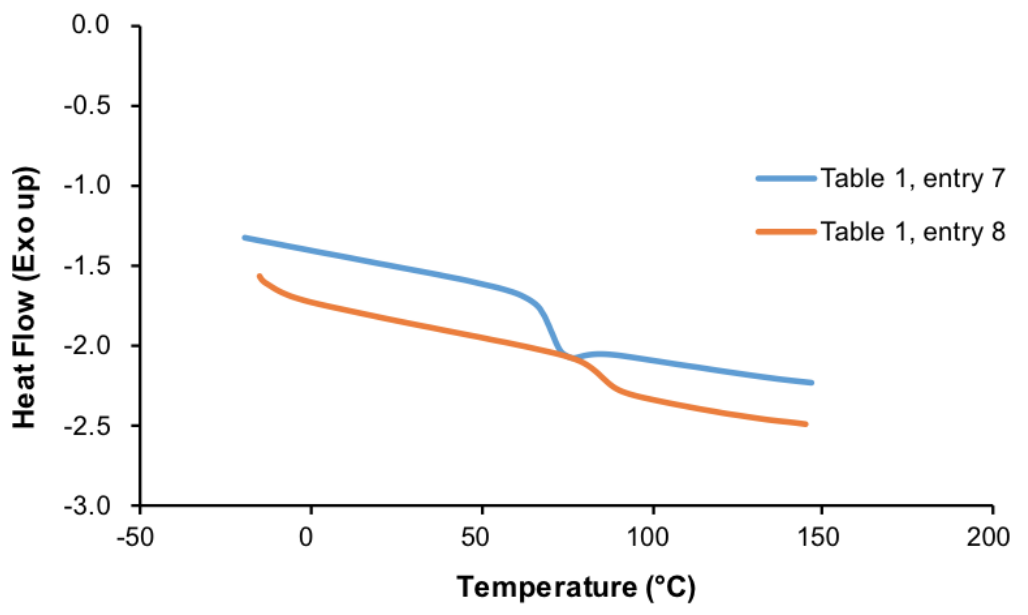

Figure S25. Overlay of DSC curves for poly(EtVCP-NHPh) in Table 1 , entry $7\left(71^{\circ} \mathrm{C}\right)$ and entry $8\left(77^{\circ} \mathrm{C}\right)$ 


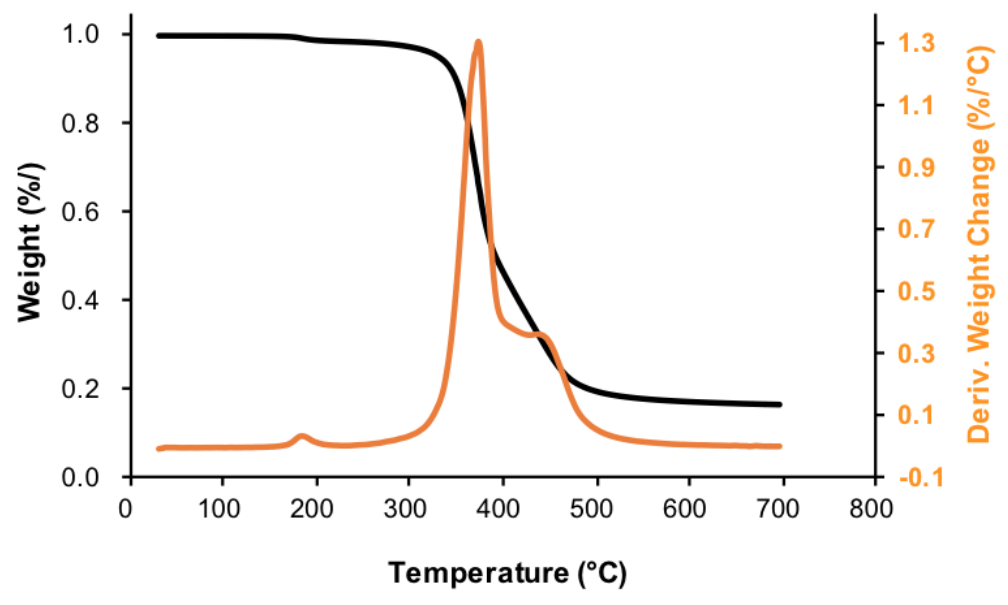

Figure S26. TGA and DTG curves for poly(EtVCP-CN) in Table 1, entry 3. $T_{\mathrm{d}}(10 \%$ weight loss) $=349{ }^{\circ} \mathrm{C} ; T_{\max }=375^{\circ} \mathrm{C}$.

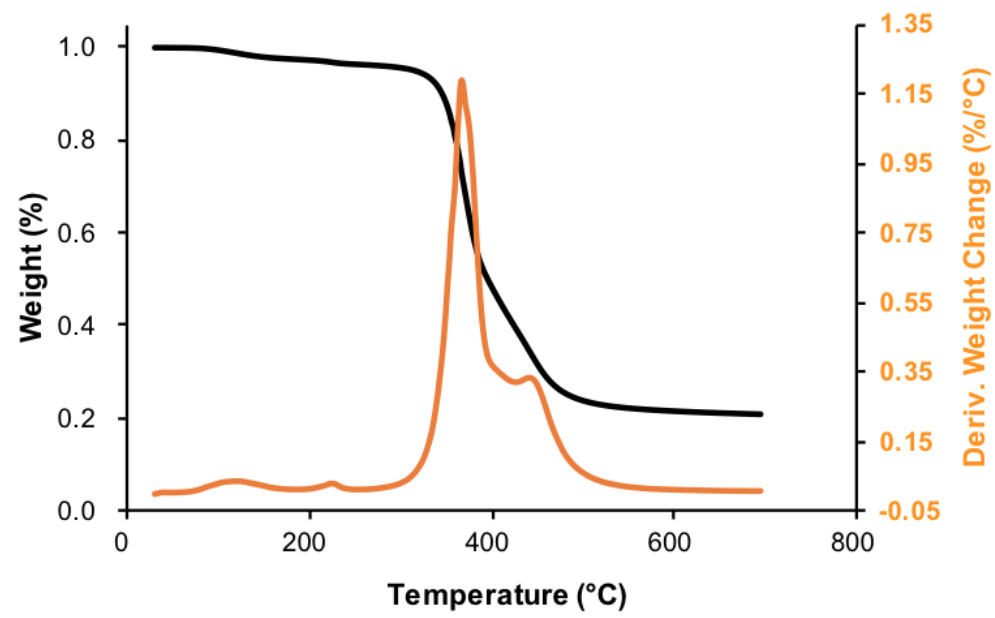

Figure S27. TGA and DTG curves for poly(EtVCP-CN) in Table 1, entry 6. $T_{\mathrm{d}}(10 \%$ weight loss) $=346{ }^{\circ} \mathrm{C} ; T_{\max }=367^{\circ} \mathrm{C}$. 


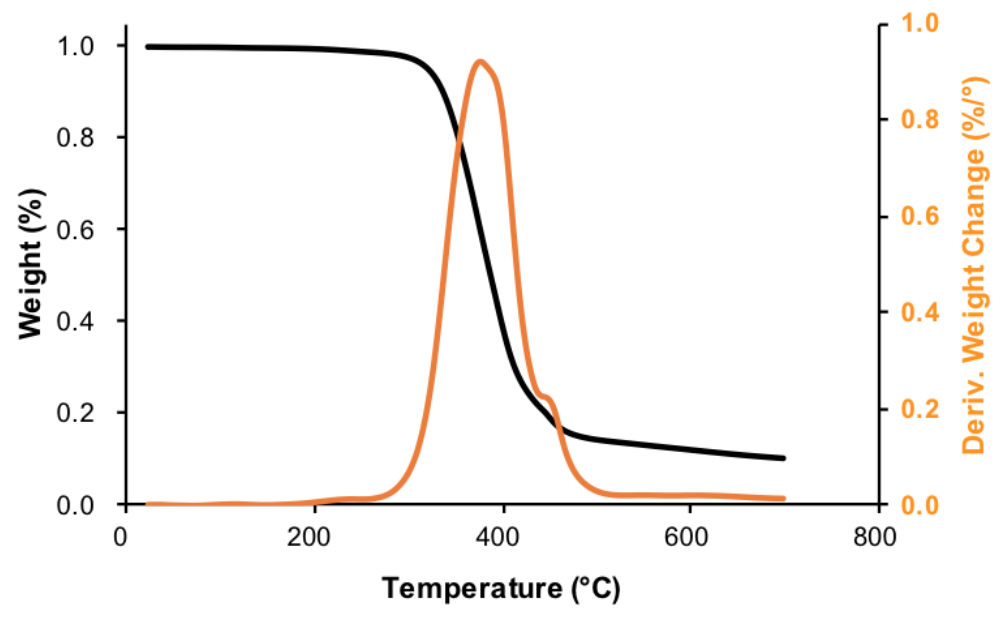

Figure S28. TGA and DTG curves for poly(EtVCP-NHPh) in Table 1, entry $7 . T_{\mathrm{d}}(10 \%$ weight loss) $=337^{\circ} \mathrm{C} ; T_{\max }=377^{\circ} \mathrm{C}$.

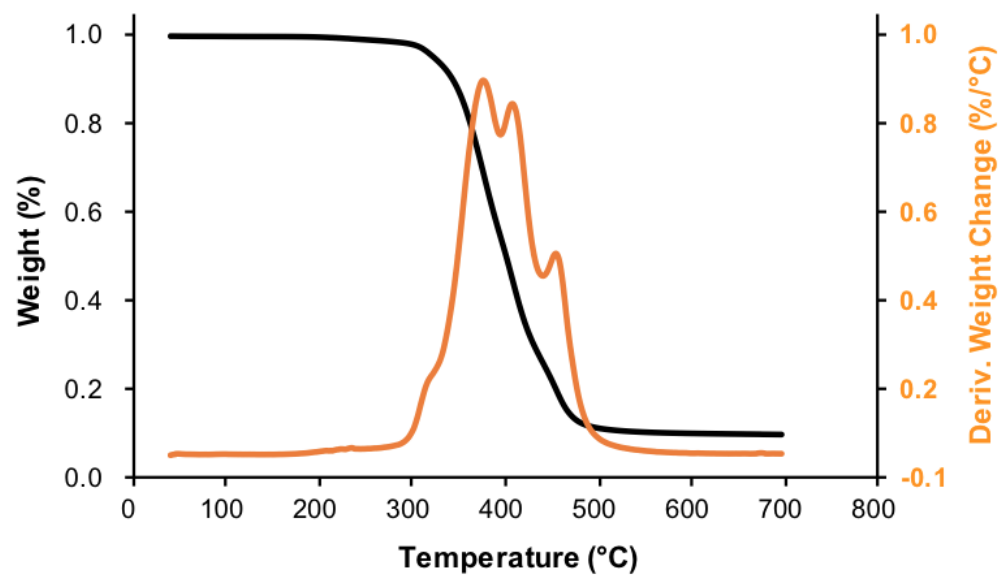

Figure S29. TGA and DTG curves for poly(EtVCP-NHPh) in Table 1, entry 8. $T_{\mathrm{d}}(10 \%$ weight loss $)=343{ }^{\circ} \mathrm{C} ; T_{\max 1}=376{ }^{\circ} \mathrm{C}, T_{\max 2}=406{ }^{\circ} \mathrm{C}, T_{\max 3}=456{ }^{\circ} \mathrm{C}$. 


\section{Polymerization of Natural Product-Derived Vinycylopropanes}

\subsection{General Experimental Procedures}

General Procedure D: $1.0 \mathrm{~mL}$ of freshly made solution $(1.0 \mathrm{mM})$ of photoredox catalyst (PC) in anhydrous dichloromethane (DCM) was added to a $20 \mathrm{~mL}$ scintillation vial equipped with a small magnetic stir bar. DCM was removed and the vial was transferred into a $\mathrm{N}_{2}$-filled glove box. To this vial, solvent, monomer $(0.5 \mathrm{mmol})$ and $0.10 \mathrm{M}$ of the initiator stock solution were sequentially added. The vial was then tightly capped and placed into a white-light LED beaker while stirring in the glove box. The temperature was about $28{ }^{\circ} \mathrm{C}$ with a cooling fan. At a given time point, a $0.10 \mathrm{~mL}$ aliquot of the reaction mixture was taken via syringe and immediately quenched by injecting into a $1.5 \mathrm{~mL}$ vial containing $\sim 0.70 \mathrm{~mL} \mathrm{CDCl}_{3}$ with $250 \mathrm{ppm}$ butylated hydroxytoluene (BHT). This aliquot was then analyzed via ${ }^{1} \mathrm{H}$ NMR for monomer conversion. The aliquot was then dried under vacuum to remove all volatiles, re-dissolved in THF and passed through a syringe filter for direct analysis by GPC. For purification, the polymerization mixture was slowly added into $30.0 \mathrm{~mL}$ of methanol (for polymerizations of EtVCP-VE, EtVCP-DA and EtVCP-C) or hexane (for polymerization of EtVCP-U) while stirring at $0{ }^{\circ} \mathrm{C}$. The precipitated polymer was collected by vacuum filtration, washed with cold methanol or hexane $(5.0 \mathrm{~mL} \times 3)$ and dried overnight under vacuum at $50{ }^{\circ} \mathrm{C}$ to a constant weight.

General Procedure E: $1.0 \mathrm{~mL}$ of freshly made solution $(1.0 \mathrm{mM})$ of photoredox catalyst (PC) in anhydrous dichloromethane (DCM) was added to a $25 \mathrm{~mL}$ Schlenk storage tube equipped with a small magnetic stir bar. DCM was removed and the tube was transferred into a $\mathrm{N}_{2}$-filled glove box. Then solvent, monomer $(0.5 \mathrm{mmol})$, and $0.10 \mathrm{M}$ DBMM stock solution were sequentially added. The Schlenk tube was then tightly capped, taken out of the glove box, then placed into pre-heat oil bath $\left(60^{\circ} \mathrm{C}\right)$ while stirring. A $34 \mathrm{~W}$ Kessil blue LED was then immediately turned on. The distance between the Schlenk tube and the blue LED was about $5 \mathrm{~cm}$. The monomer conversion and $S_{\mathrm{L}}$ were determined by crude ${ }^{1} \mathrm{H}-\mathrm{NMR}$ while $M_{\mathrm{n}}$ and $Ð$ of the obtained polymer was determined by the GPC. Purification of the polymer followed the procedure described in General Procedure D. 


\subsection{Optimization for Polymerization of EtVCP-VE}

Table S5. Initiator screening for polymerization of EtVCP-VE ${ }^{a}$

\begin{tabular}{ccccccc}
\hline Entry & Initiator & Conv. $(\boldsymbol{\%})$ & $\boldsymbol{M}_{\mathbf{n}}(\mathbf{k D a})$ & $\boldsymbol{D}\left(\boldsymbol{M}_{\mathbf{w}} / \boldsymbol{M}_{\mathbf{n}}\right)$ & $\boldsymbol{I}^{*}(\boldsymbol{\%})$ & $\boldsymbol{S}_{\mathbf{L}}(\boldsymbol{\%})$ \\
1 & DBMM & 96 & 31.6 & 1.29 & 91 & 96 \\
2 & EBP & 80 & 31.8 & 1.48 & 76 & 96 \\
3 & M2BP & 66 & 27.9 & 1.41 & 72 & 96 \\
\hline
\end{tabular}

${ }^{a}$ Polymerizations of [EtVCP-VE]/[initiator]/[1] (500/10/1) were performed in $1.0 \mathrm{~mL}$ of anhydrous EtOAc for $6 \mathrm{~h}$, with white LEDs irradiation at $28^{\circ} \mathrm{C}$.

Table S6. Solvent screening for polymerization of $\mathrm{EtVCP}-\mathrm{VE}^{a}$

\begin{tabular}{ccccccc}
\hline Entry & Solvent & Conv. $(\boldsymbol{\%})$ & $\boldsymbol{M}_{\mathbf{n}}(\mathbf{k D a})$ & $\boldsymbol{D}\left(\boldsymbol{M}_{\mathbf{w}} / \boldsymbol{M}_{\mathbf{n}}\right)$ & $\boldsymbol{I}^{*}(\boldsymbol{\%})$ & $\boldsymbol{S}_{\mathbf{L}}(\boldsymbol{\%})$ \\
1 & DMAc & 79 & 27.2 & 1.44 & 88 & 96 \\
2 & EtOAc & 96 & 31.6 & 1.29 & 91 & 96 \\
4 & $\mathrm{PhCl}$ & 94 & 27.6 & 1.23 & 103 & 96 \\
\hline
\end{tabular}

${ }^{a}$ Polymerizations of [EtVCP-VE]/[DBMM]/[1] (500/10/1) were performed in $1.0 \mathrm{~mL}$ of anhydrous solvent for $6 \mathrm{~h}$, with white LEDs irradiation at $28^{\circ} \mathrm{C}$. DCE $=1,2$-dichloroethane.

Table S7. Control experiments ${ }^{a}$

\begin{tabular}{ccccccccc}
\hline Entry & Light & PC 1 & DBMM & $\begin{array}{c}\text { Conv. } \\
(\%)\end{array}$ & $\begin{array}{c}M_{\mathrm{n}(\mathrm{GPC})} \\
(\mathrm{kDa})\end{array}$ & $\begin{array}{c}Ð \\
\left(M_{\mathrm{w}} / M_{\mathrm{n}}\right)\end{array}$ & $I^{*}(\%)$ & $S_{\mathrm{L}}(\%)$ \\
1 & - & - & - & 0 & - & - & - & - \\
2 & - & $\sqrt{ }$ & - & 0 & - & - & - & - \\
3 & - & - & $\sqrt{ }$ & 0 & - & - & - & - \\
4 & - & $\sqrt{ }$ & $\sqrt{ }$ & 0 & - & - & - & - \\
5 & $\sqrt{ }$ & - & - & 0 & - & - & - & - \\
6 & $\sqrt{ }$ & $\sqrt{ }$ & - & 0 & - & - & - & - \\
7 & $\sqrt{ }$ & - & $\sqrt{ }$ & 0 & - & - & - & - \\
\hline
\end{tabular}

${ }^{a}$ Polymerizations of $[\mathrm{EtVCP}-\mathrm{VE}] /[\mathrm{DBMM}] /[1]$ (500/10/1) were performed in $1.0 \mathrm{~mL}$ of anhydrous EtOAc for $12 \mathrm{~h}$, with white LEDs irradiation at $28^{\circ} \mathrm{C}$. 


\subsection{Kinetic Study and Pulsed-Irradiation Experiment}

Table S8. Progress analysis for polymerization of EtVCP-VE at $28{ }^{\circ} \mathrm{C}$ with white $\mathrm{LED}^{a}$

\begin{tabular}{cccccccc}
\hline \multirow{2}{*}{ Entry } & Time (h) & \multicolumn{2}{c}{ Conv. (\%) } & $\boldsymbol{M}_{\mathbf{n}}(\mathbf{k D a})$ & $\boldsymbol{D}\left(\boldsymbol{M}_{\mathbf{w}} / \boldsymbol{M}_{\mathbf{n}}\right)$ & $\boldsymbol{I}^{*}(\boldsymbol{\%})$ & $\boldsymbol{S}_{\mathbf{L}}(\boldsymbol{\%})$ \\
1 & 0.5 & 25 & 0 & 10.2 & 1.21 & 75 & 92 \\
2 & 1 & 51 & 1 & 17.0 & 1.28 & 93 & 97 \\
3 & 2 & 77 & 2 & 24.3 & 1.34 & 98 & 96 \\
4 & 4 & 86 & 4 & 27.3 & 1.33 & 99 & 96 \\
5 & 5 & 88 & 6 & 29.9 & 1.27 & 96 & 95 \\
\hline
\end{tabular}

${ }^{a}$ The polymerization of $[\mathrm{EtVCP}-\mathrm{VE}] /[\mathrm{DBMM}] /[1](500 / 10 / 1)$ was performed in $1.0 \mathrm{~mL}$ of anhydrous EtOAc, with white LED irradiation at $28^{\circ} \mathrm{C}$.

Note: The trans-EtVCP-VE was polymerized faster than cis-EtVCP-VE due to steric hindrance. However, this does not affect the intramolecular radical cyclization preference due to faster radical racemization after the ring-opening event.

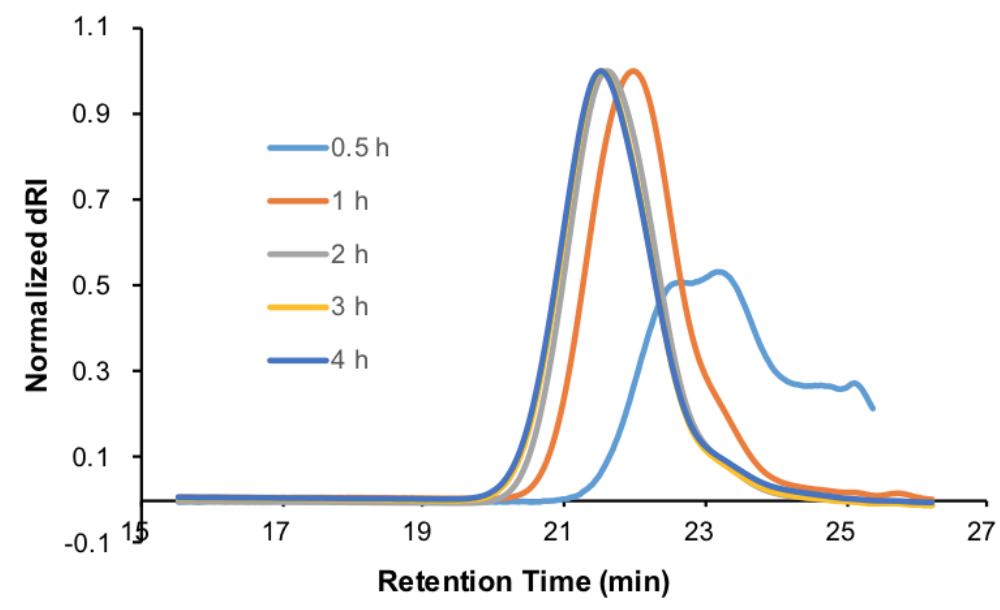

Figure S30. Overlay of GPC traces for poly(EtVCP-VE) in Table S8. 
Table S9. Pulsed-irradiation experiment for polymerization of EtVCP-VE at $28{ }^{\circ} \mathrm{C}$ with white $\mathrm{LED}^{a}$

\begin{tabular}{ccccccc}
\hline Entry & Time $(\mathbf{h})$ & Conv. $(\boldsymbol{\%})$ & $\boldsymbol{M}_{\mathbf{n}}(\mathbf{k D a})$ & $\boldsymbol{D}\left(\boldsymbol{M}_{\mathbf{w}} / \boldsymbol{M}_{\mathbf{n}}\right)$ & $\boldsymbol{I}^{*}(\boldsymbol{\%})$ & $\boldsymbol{S}_{\mathbf{L}}(\boldsymbol{\%})$ \\
1 & 0.5 & 26 & 14.1 & 1.44 & 57 & 91 \\
2 & 1.5 & 26 & 14.0 & 1.51 & 57 & 91 \\
3 & 3 & 72 & 24.5 & 1.31 & 89 & 96 \\
4 & 15 & 72 & 24.3 & 1.32 & 90 & 96 \\
5 & 16 & 86 & 26.9 & 1.32 & 97 & 96 \\
6 & 20 & 86 & 26.8 & 1.32 & 97 & 96 \\
\hline
\end{tabular}

${ }^{a}$ The polymerization of [EtVCP-VE]/[DBMM]/[1] (500/10/1) was performed in $1.0 \mathrm{~mL}$ of anhydrous EtOAc, with white LED irradiation at $28^{\circ} \mathrm{C}$.

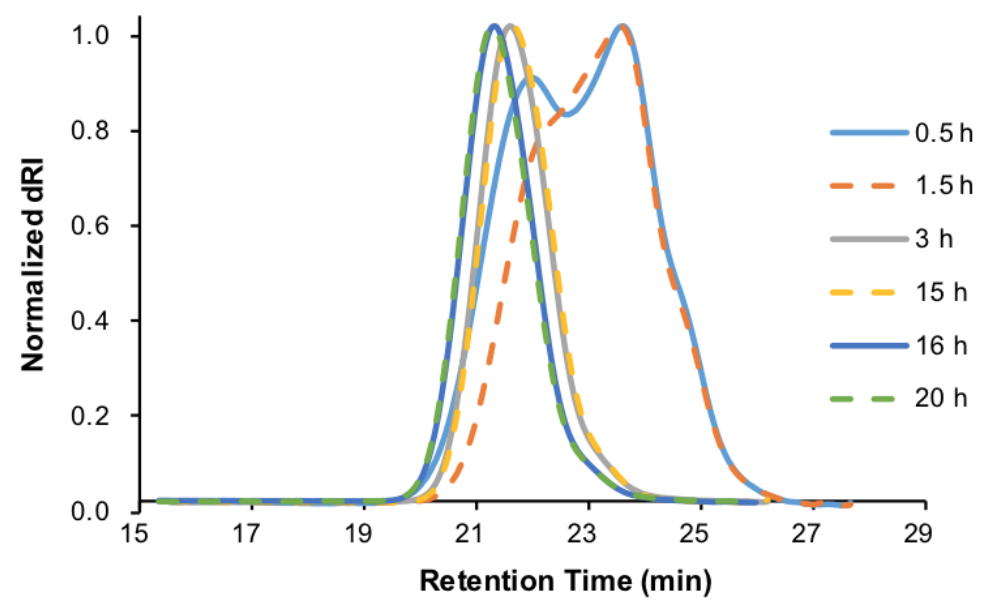

Figure S31. Overlay of GPC traces for poly(EtVCP-VE) in Table S9. 


\subsection{Chain-Extension Experiment}

Poly(EtVCP-VE) macroinitiator (13.0 kDa, $\left.D=1.15, S_{\mathrm{L}}=92 \%\right)$ was synthesized and purified according to General Procedure $\mathbf{C}(1.0 \mathrm{mmol}$ scale, $[\mathrm{M}]:[\mathrm{DBMM}]:[1]=$ 250:10:1).

An oven-dried $20 \mathrm{~mL}$ scintillation vial was charged with a magnetic stir bar, the isolated poly(EtVCP) macroinitiator $(130.0 \mathrm{mg}, 0.010 \mathrm{mmol})$ and PC $1(0.44 \mathrm{mg}, 0.0010$ mmol). The scintillation vial was transferred into a $\mathrm{N}_{2}$-filled glovebox where $1.0 \mathrm{~mL}$ of anhydrous $\mathrm{PhCl}$ and EtVCP-C2 (166.7 $\mathrm{mg}, 0.25 \mathrm{mmol})$ were quickly added. The vial was then tightly capped and irradiated white LED while stirring in the glove box. After $12 \mathrm{~h}$, a $0.10 \mathrm{~mL}$ aliquot was taken and quenched by injecting into a vial containing $\sim 0.70 \mathrm{~mL}$ $\mathrm{CDCl}_{3}$ with $250 \mathrm{ppm}$ butylated hydroxytoluene (BHT). This aliquot was then analyzed via ${ }^{1} \mathrm{H}$ NMR for conversion of monomer to polymer ( $88 \%$ conv. was achieved in this reaction). The aliquot was then dried under vacuum for direct analysis by GPC equipped with multiangle light scattering $\left(M_{\mathrm{n}}=31.6 \mathrm{kDa}, Ð=1.39, I^{*}=91 \%, S_{\mathrm{L}}=84 \%\right)$.

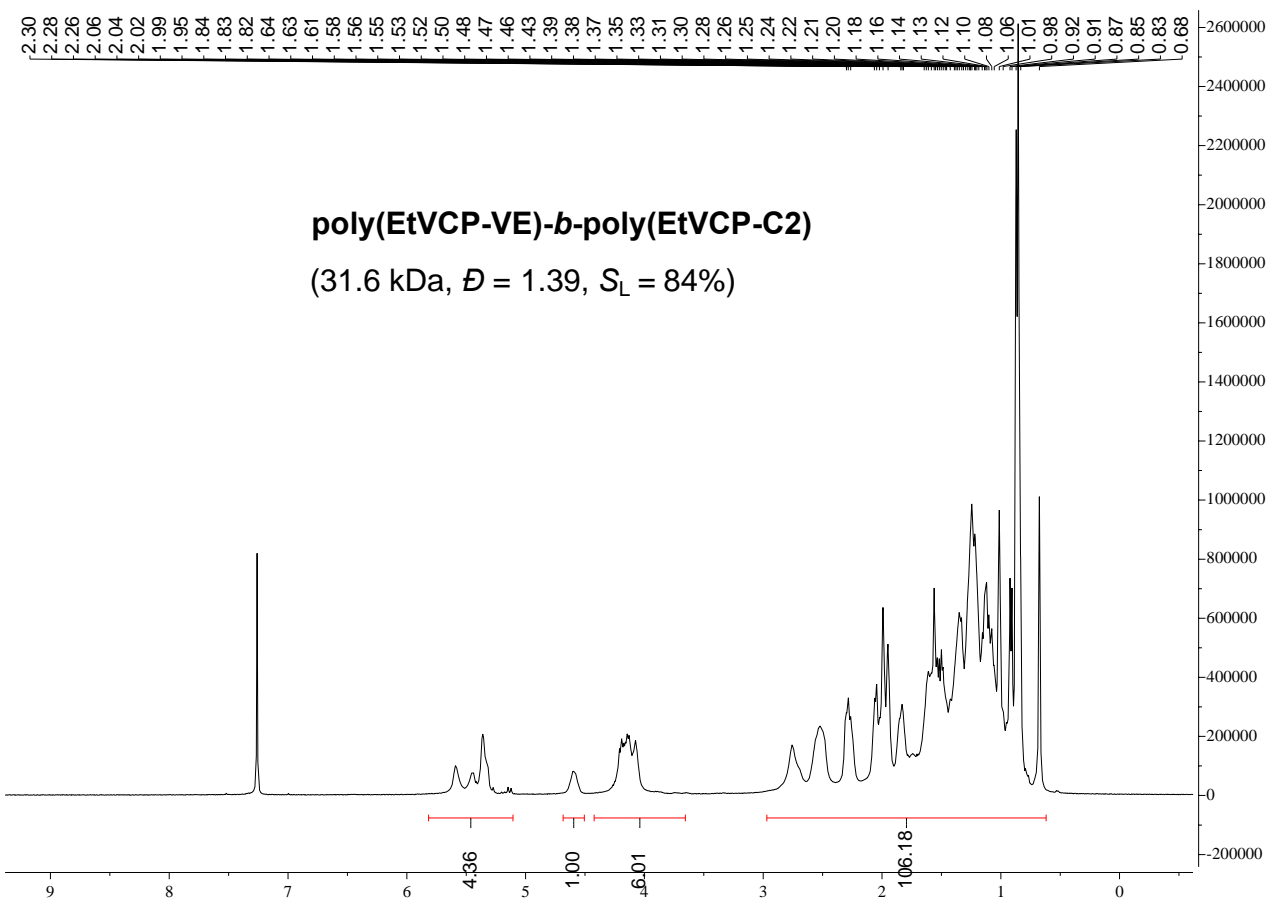

Figure S32. ${ }^{1} \mathrm{H}-\mathrm{NMR}$ spectra of poly(EtVCP-VE)- $b$-poly(EtVCP-C2). 


\section{5 ${ }^{1}$ H-NMR Spectrum of Natural Product-Derived Poly(vinylcyclopropanes)}

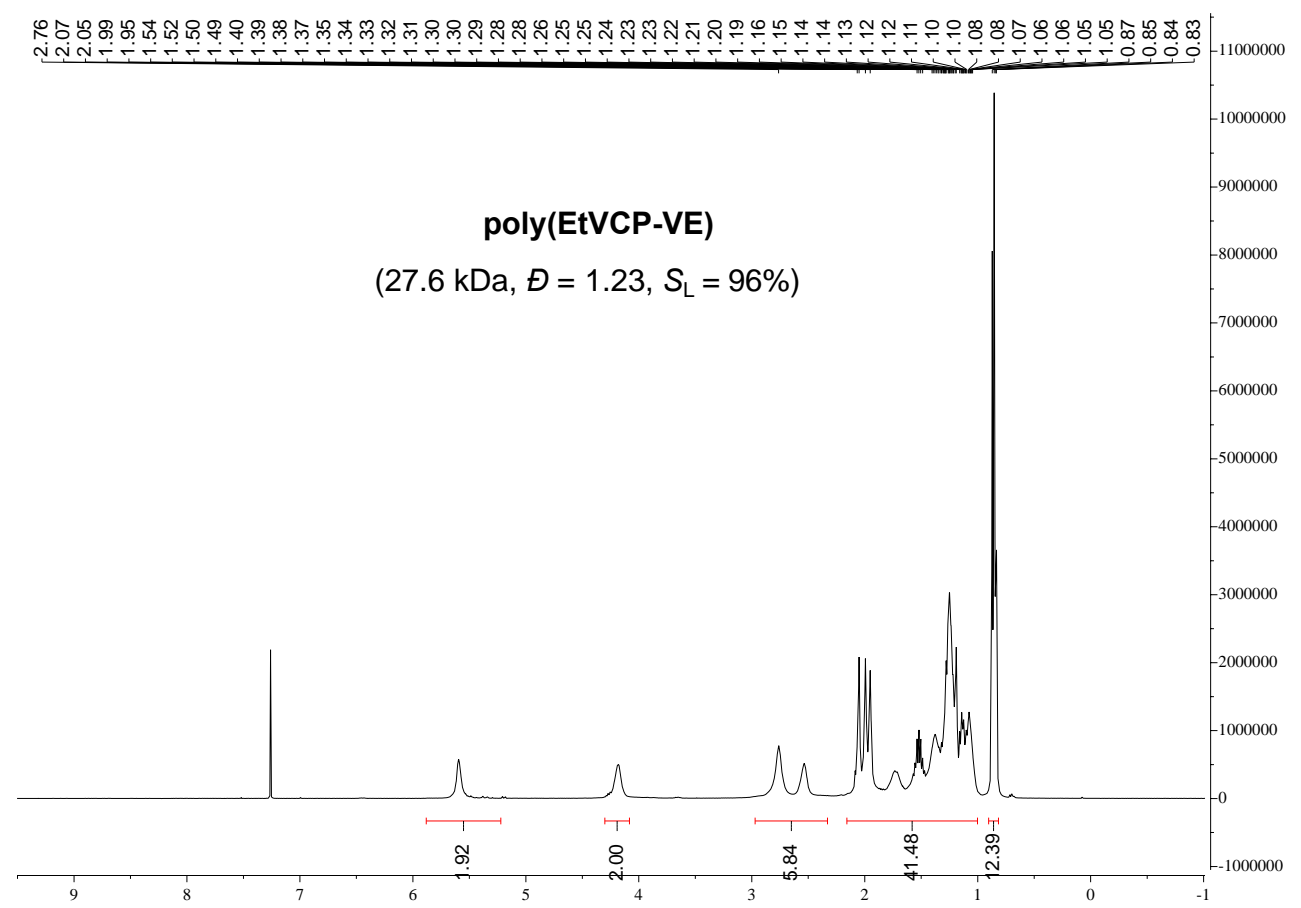

Figure S33. ${ }^{1} \mathrm{H}-\mathrm{NMR}$ spectra of poly(EtVCP-VE) in Table 2, entry 4.

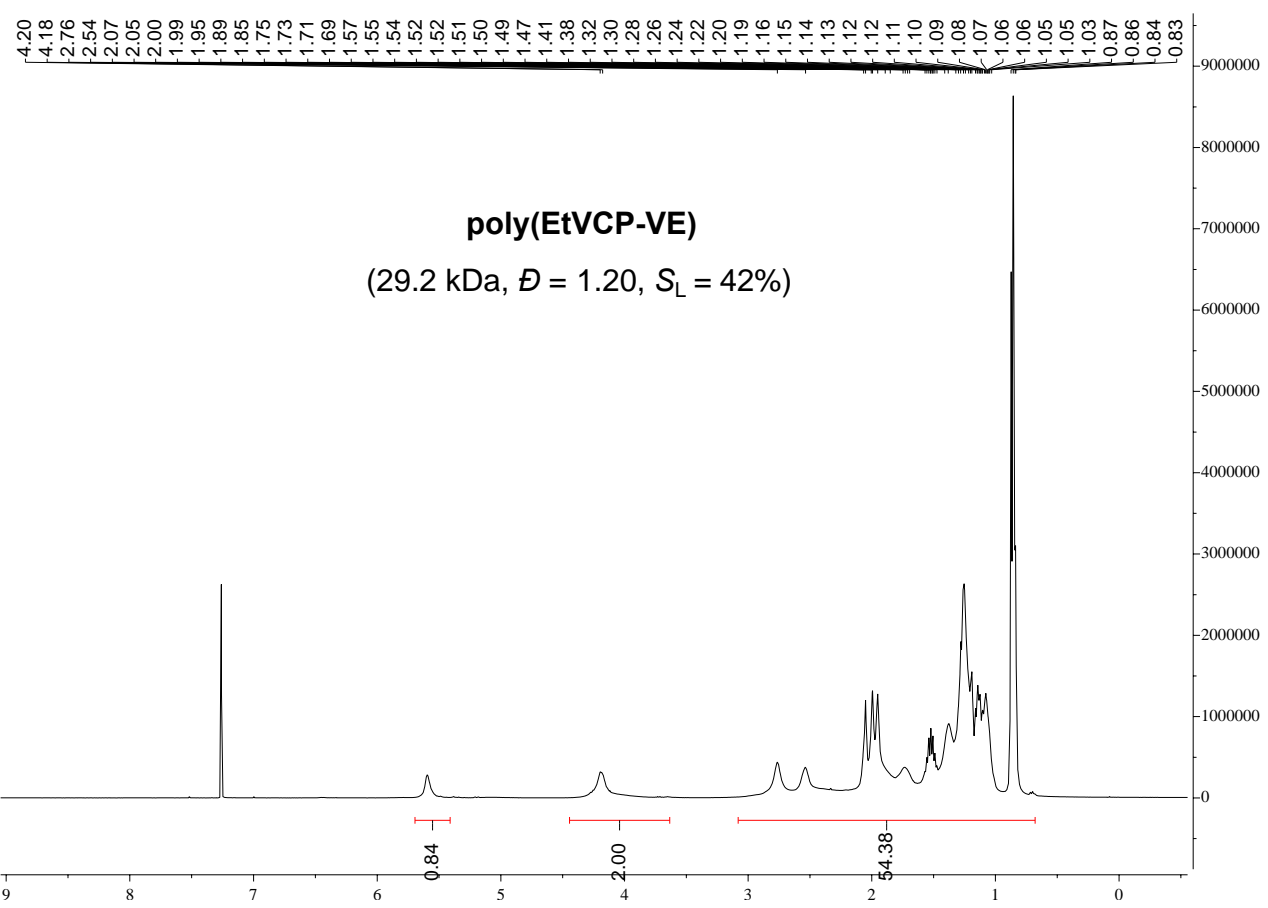

Figure S34. ${ }^{1} \mathrm{H}-\mathrm{NMR}$ spectra of poly(EtVCP-VE) in Table 3, entry 3. 


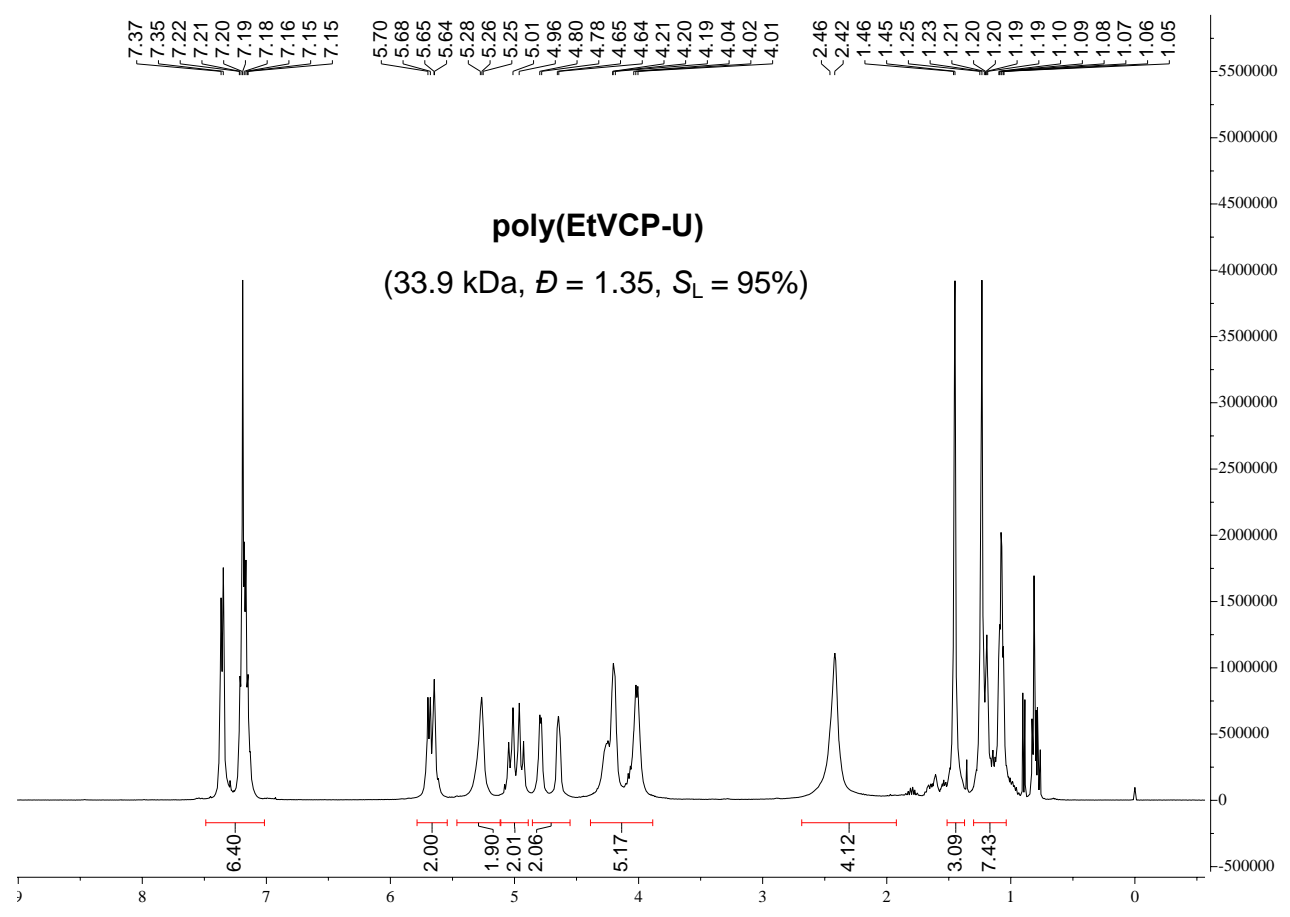

Figure S35. ${ }^{1} \mathrm{H}-\mathrm{NMR}$ spectra of poly(EtVCP-U) in Table 2, entry 13.

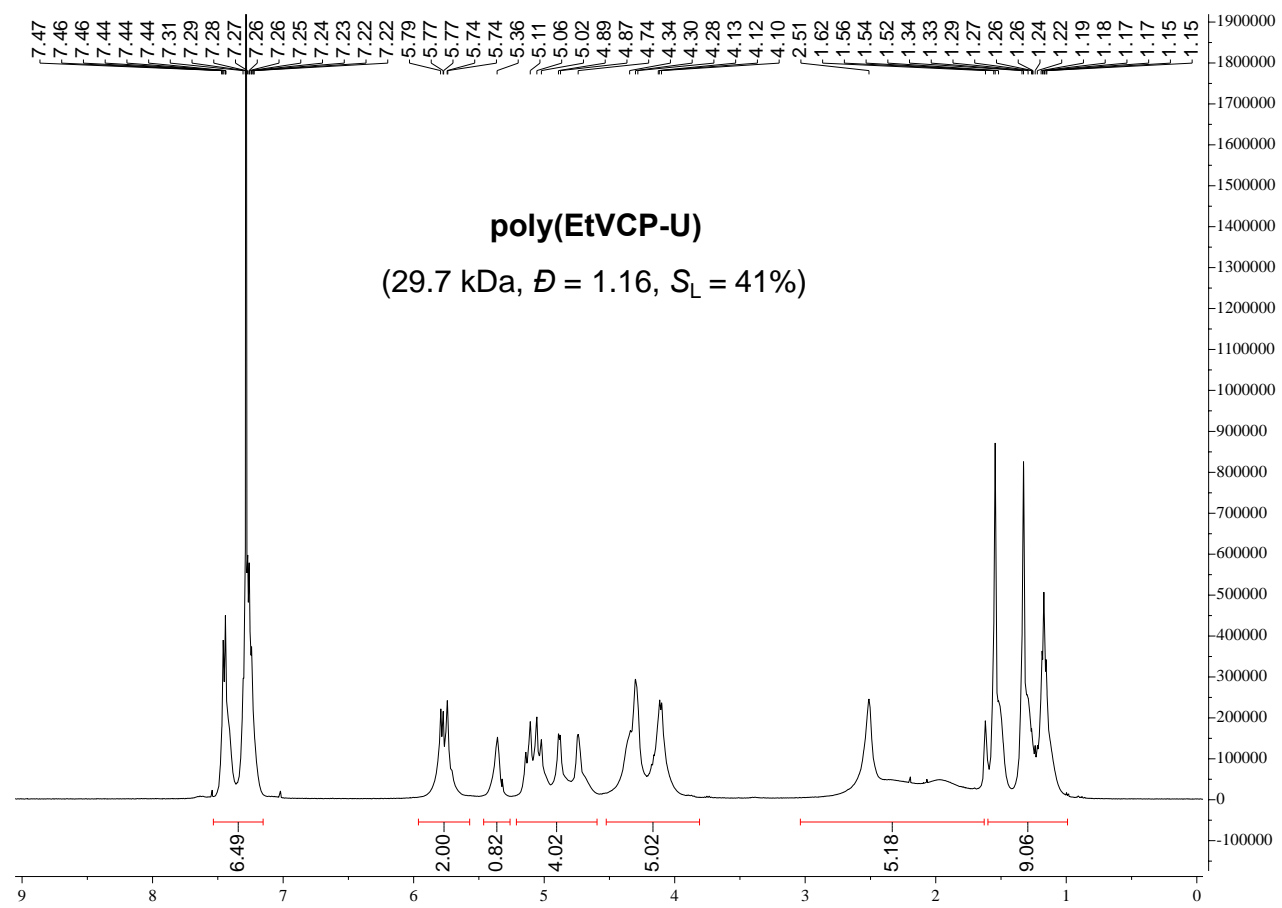

Figure S36. ${ }^{1} \mathrm{H}-\mathrm{NMR}$ spectra of poly(EtVCP-U) in Table 3, entry 4. 


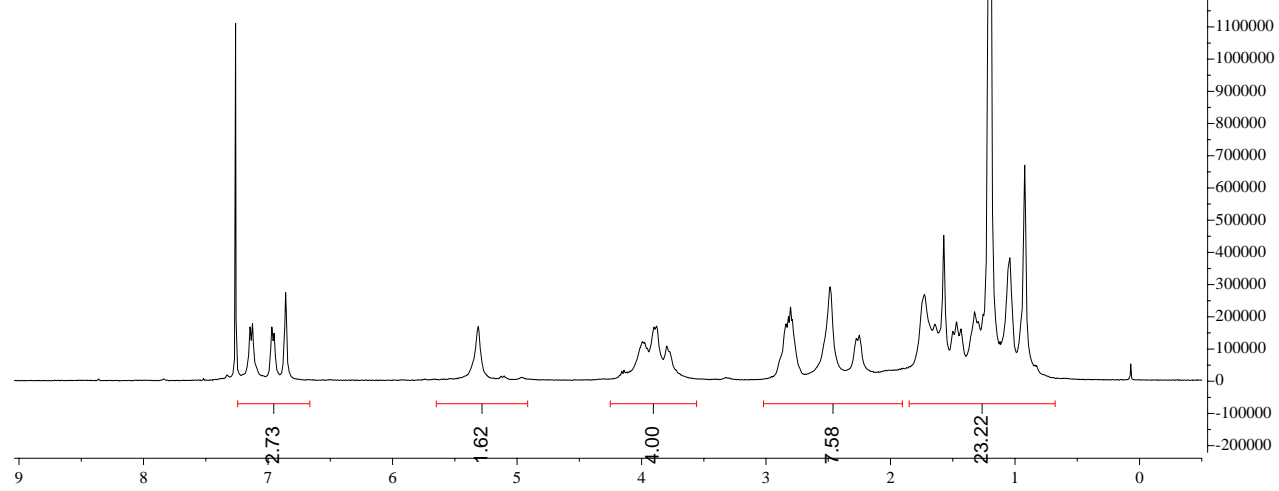

Figure S37. ${ }^{1} \mathrm{H}-\mathrm{NMR}$ spectra of poly(EtVCP-DA) in Table 2, entry 14.

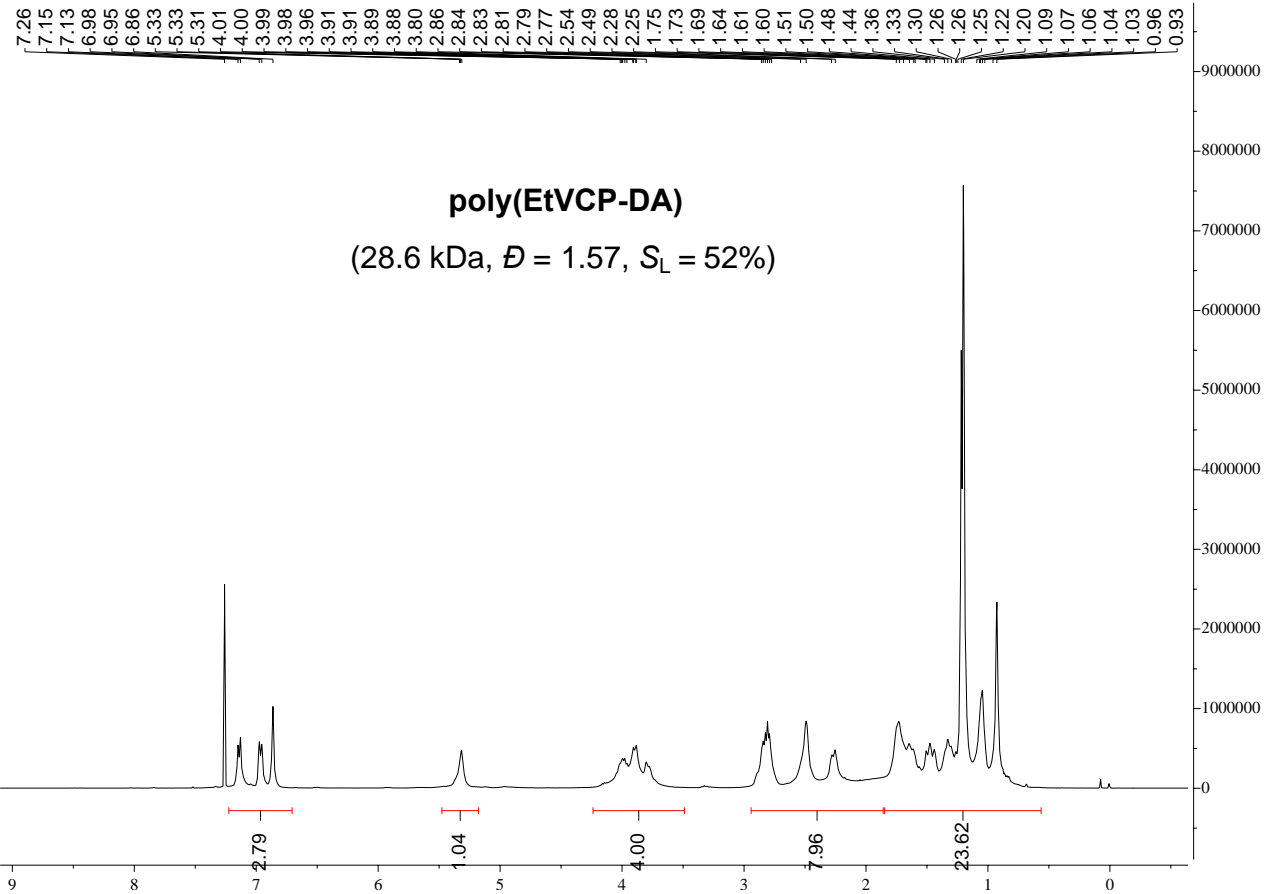

Figure S38. ${ }^{1} \mathrm{H}-\mathrm{NMR}$ spectra of poly(EtVCP-DA) in Table 3, entry 6. 


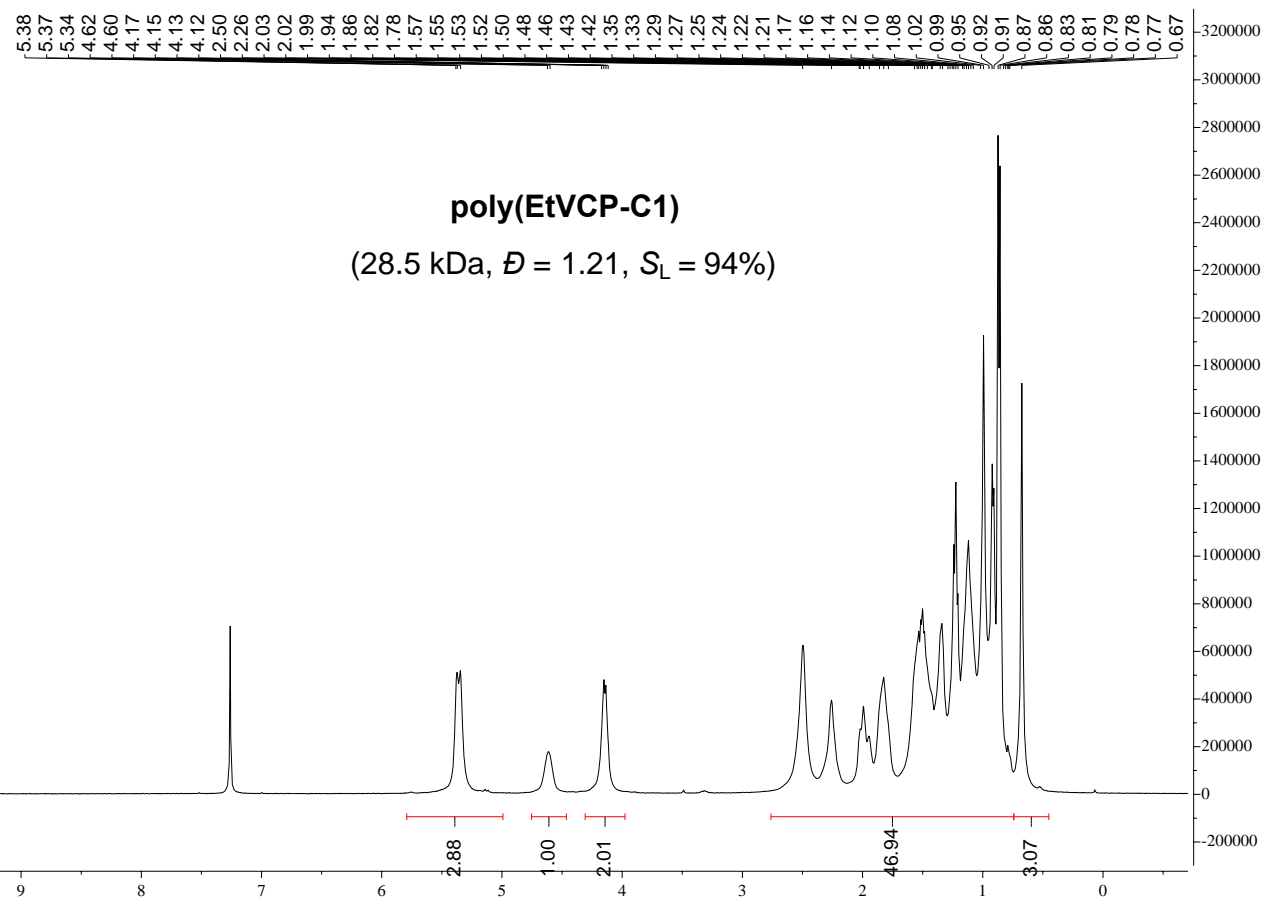

Figure S39. ${ }^{1} \mathrm{H}-\mathrm{NMR}$ spectra of poly(EtVCP-C1) in Table 2, entry 17.

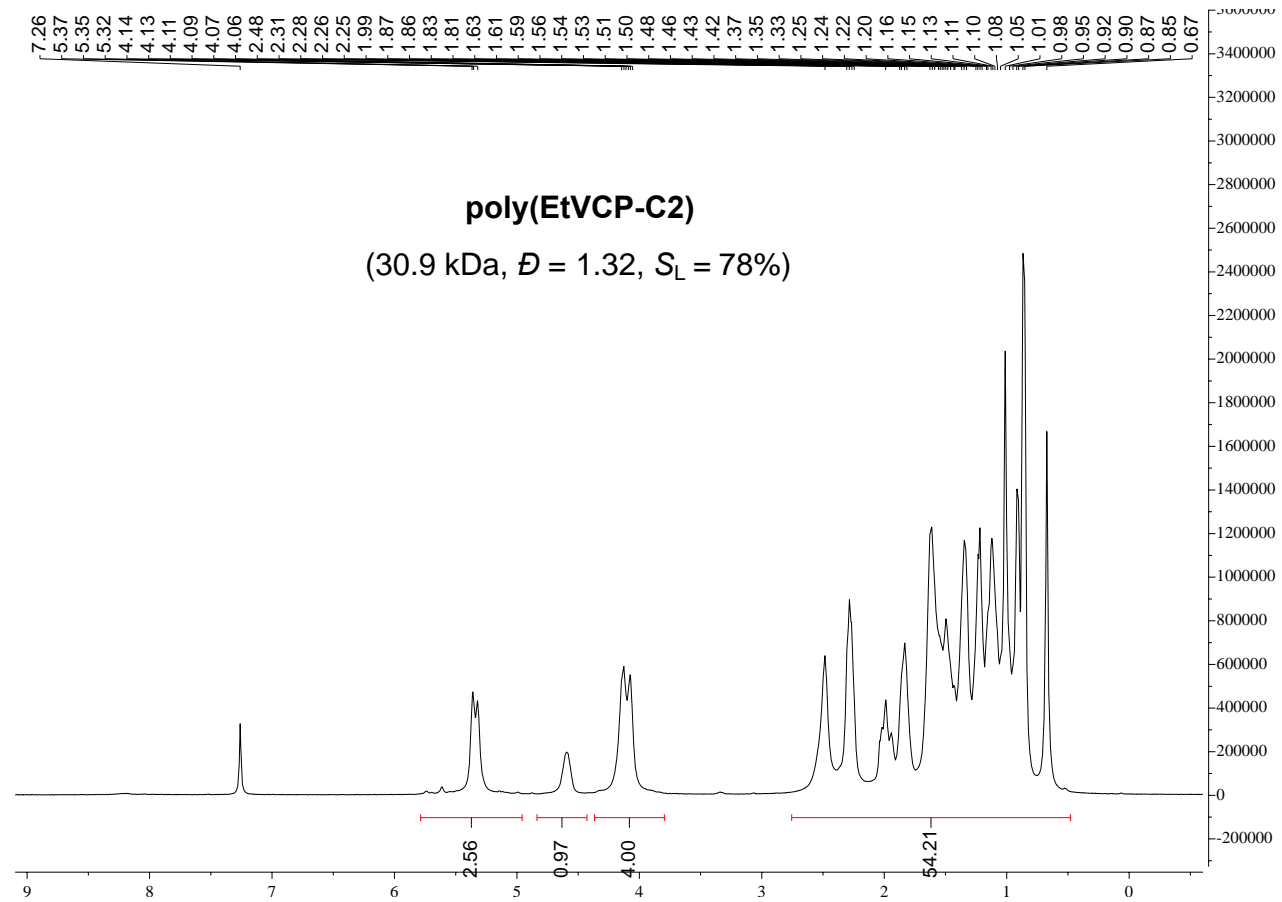

Figure S40. ${ }^{1} \mathrm{H}-\mathrm{NMR}$ spectra of poly(EtVCP-C2) in Table 2, entry 18. 


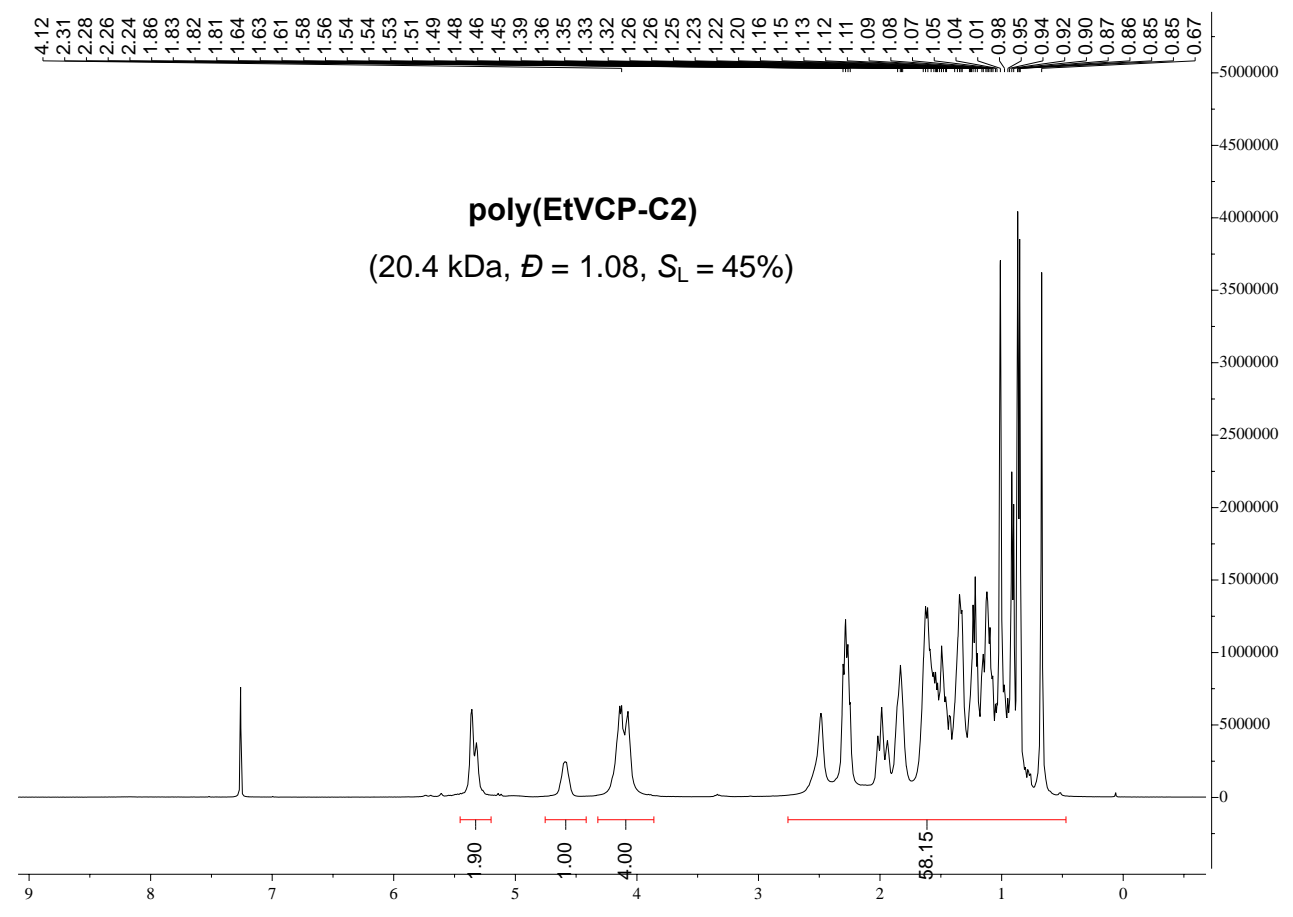

Figure S41. ${ }^{1} \mathrm{H}-\mathrm{NMR}$ spectra of poly(EtVCP-C2) in Table 3, entry 11. 


\subsection{GPC Traces of Natural Product-Derived Poly(vinylcyclopropanes)}

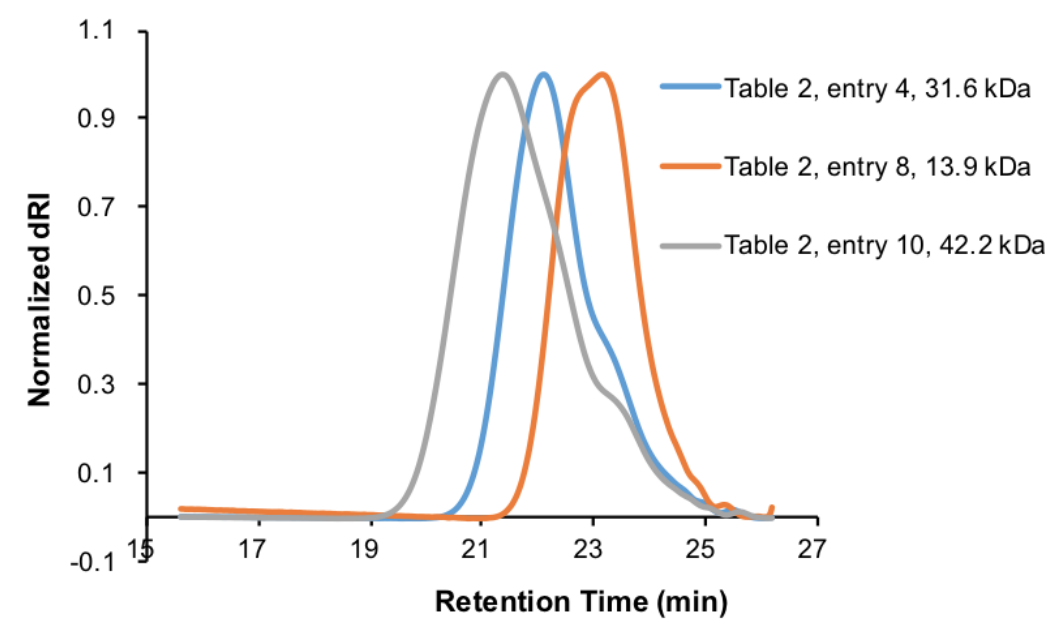

Figure S42. GPC traces of poly(EtVCP-VE) in Table 2.

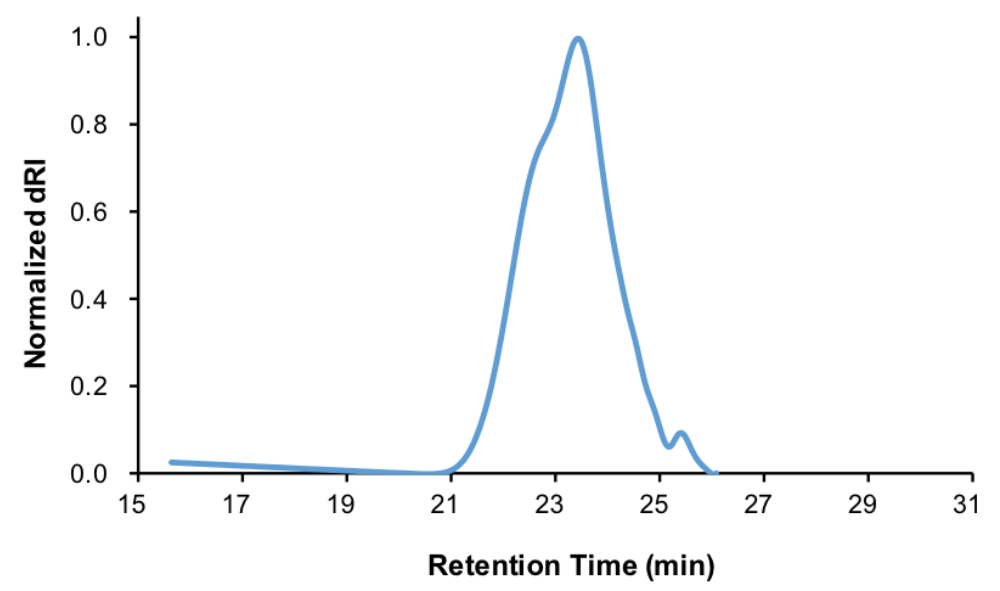

Figure S43. GPC traces of poly(EtVCP-VE) in Table 3, entry $3\left(29.2 \mathrm{kDa}, Ð=1.20, S_{\mathrm{L}}=\right.$ $42 \%)$. 


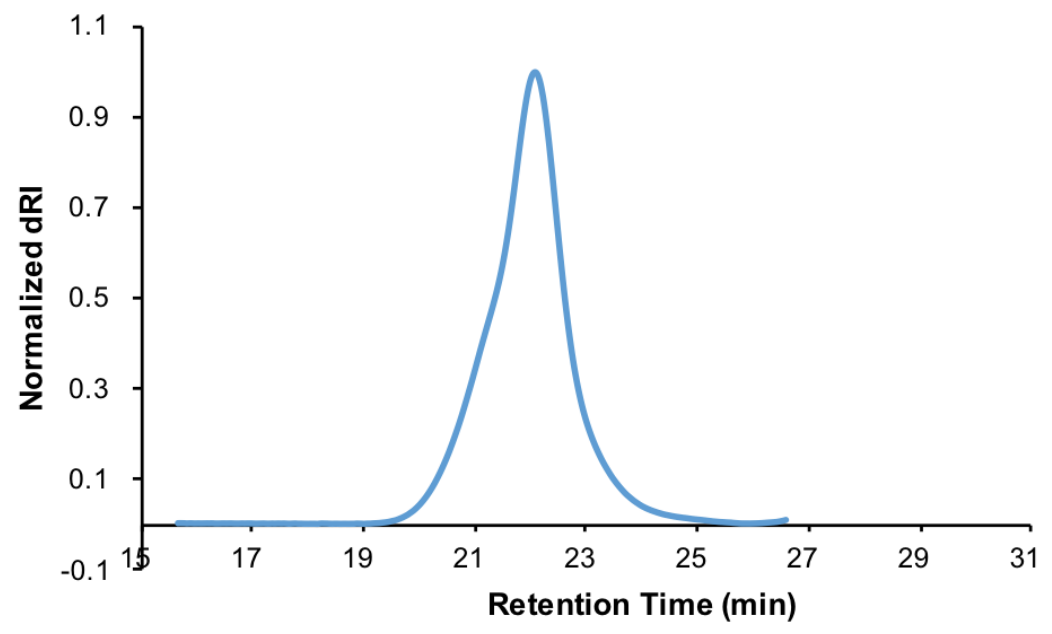

Figure S44. GPC traces of poly(EtVCP-U) in Table 2, entry $13\left(33.9 \mathrm{kDa}, Ð=1.35, S_{\mathrm{L}}=\right.$ $95 \%)$.

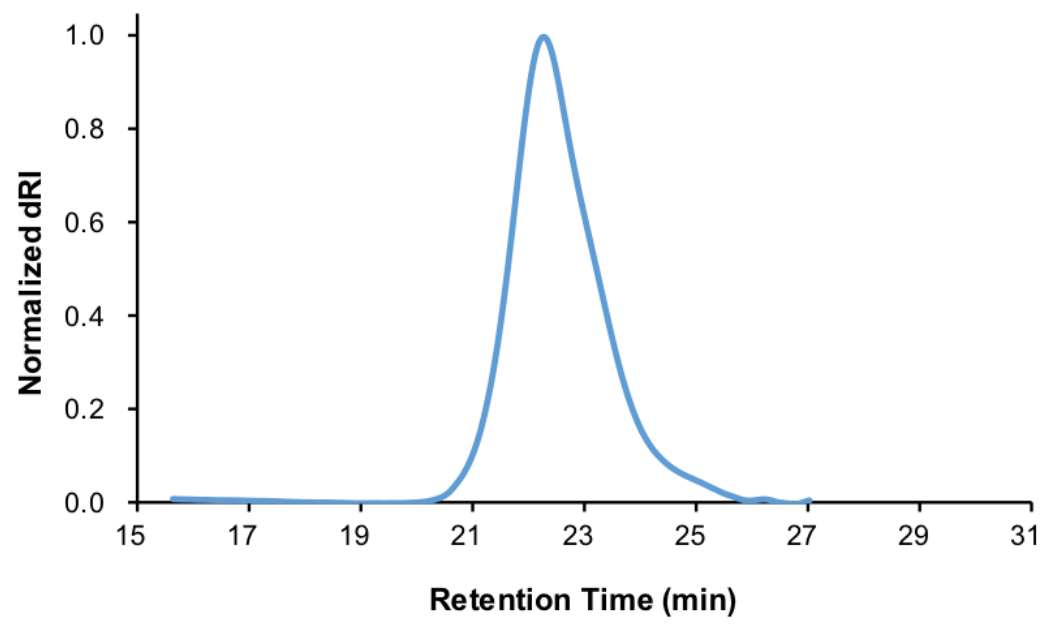

Figure S45. GPC traces of poly(EtVCP-U) in Table 3, entry $4\left(29.7 \mathrm{kDa}, Ð=1.16, S_{\mathrm{L}}=\right.$ $41 \%)$. 


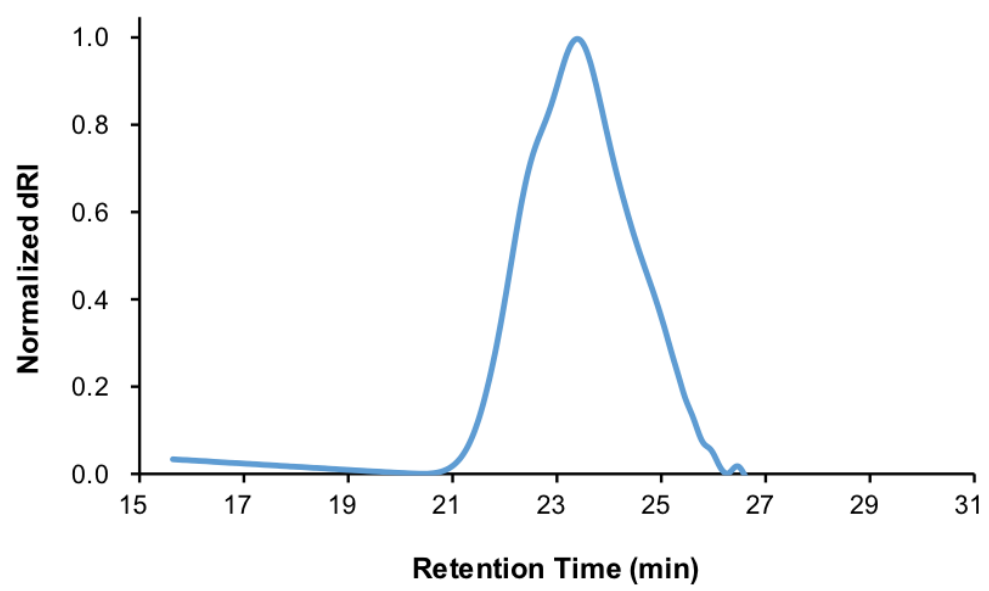

Figure S46. GPC traces of poly(EtVCP-DA) in Table 2, entry $14\left(21.6 \mathrm{kDa}, Ð=1.51, S_{\mathrm{L}}\right.$ $=81 \%$ ).

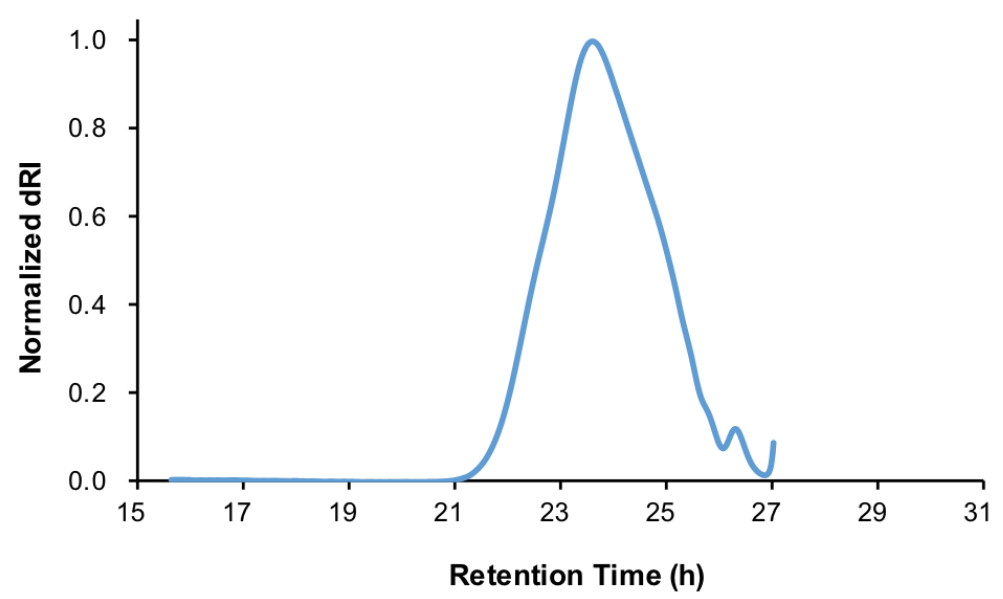

Figure S47. GPC traces of poly(EtVCP-DA) in Table 3 , entry $6\left(28.6 \mathrm{kDa}, Ð=1.57, S_{\mathrm{L}}=\right.$ $52 \%)$. 


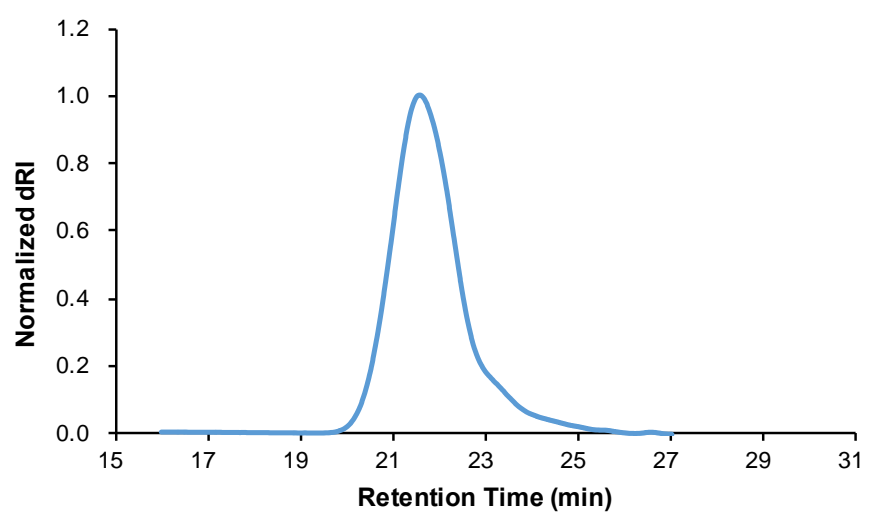

Figure S48. GPC traces of poly(EtVCP-C1) in Table 2, entry $17\left(28.5 \mathrm{kDa}, \emptyset=1.21, S_{\mathrm{L}}\right.$ =94\%).

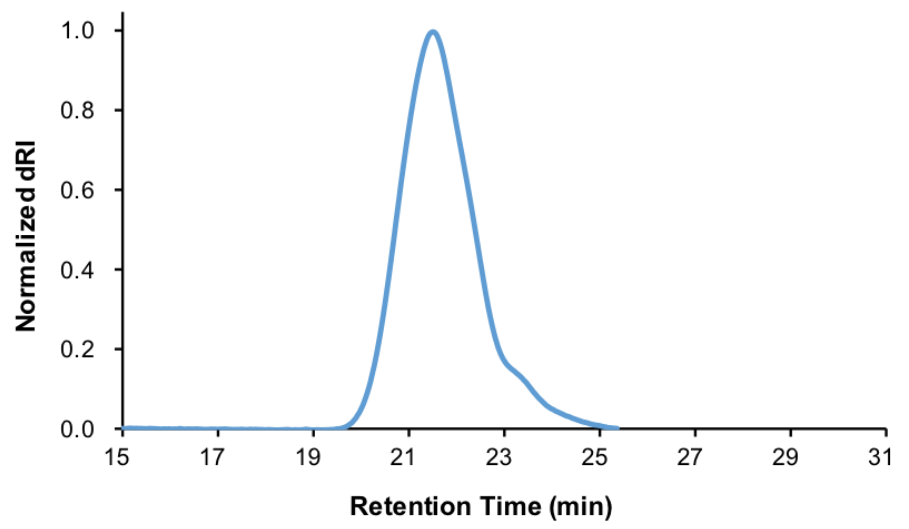

Figure S49. GPC traces of poly(EtVCP-C) in Table 2, entry $18\left(30.9 \mathrm{kDa}, Ð=1.32, S_{\mathrm{L}}=\right.$ $78 \%$ ).

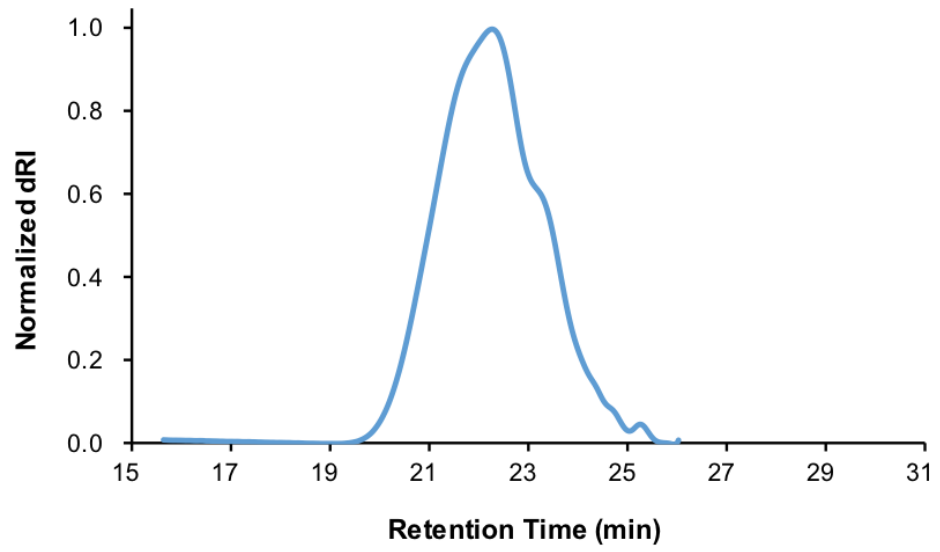

Figure S50. GPC traces of poly(EtVCP-C) in Table 3, entry $11\left(20.4 \mathrm{kDa}, Ð=1.08, S_{\mathrm{L}}=\right.$ $45 \%)$. 


\subsection{DSC and TGA Curves of Natural Product-Derived Poly(vinylcyclopropanes)}

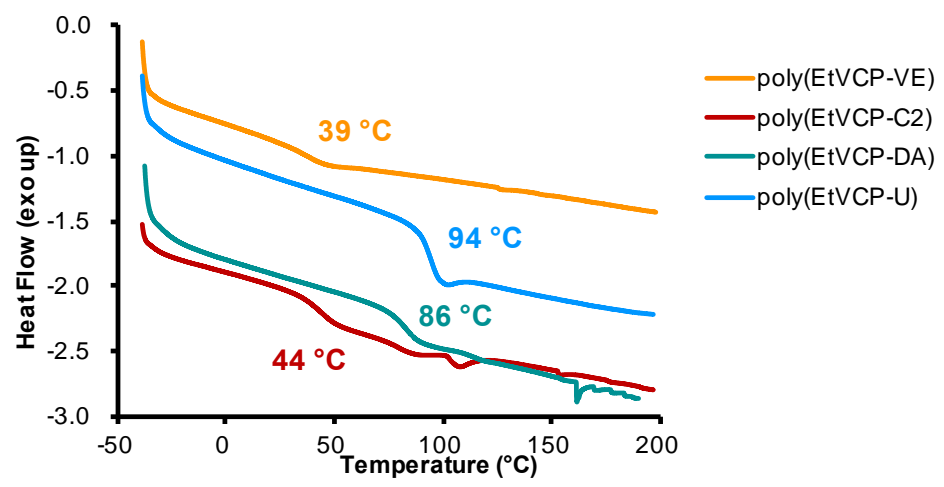

Figure S51. Overlay of DSC curves of poly(EtVCP-VE), poly(EtVCP-U), poly(EtVCPDA), and poly(EtVCP-C2) in Table 2, entries 4, 13, 14, and 18.

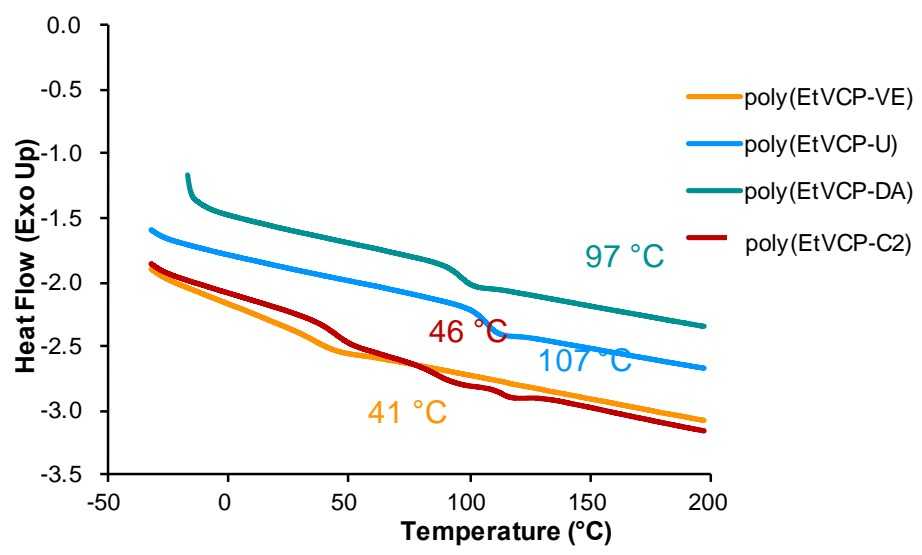

Figure S52. Overlay of DSC curves of poly(EtVCP-VE), poly(EtVCP-U), poly(EtVCPDA), and poly(EtVCP-C2) in Table 3, entries 3, 4, 6, and 11.

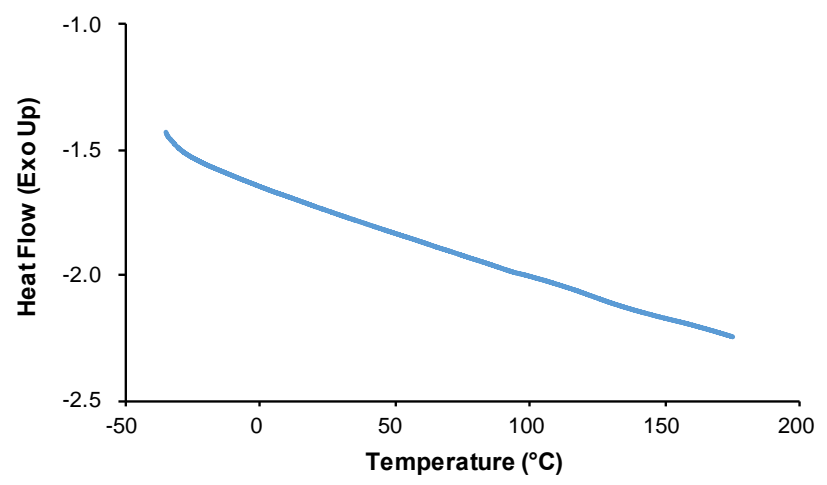

Figure S53. DSC curve of poly(EtVCP-C1) in Table 2, entries 17. No obvious $T_{\mathrm{g}}$ was observed. 


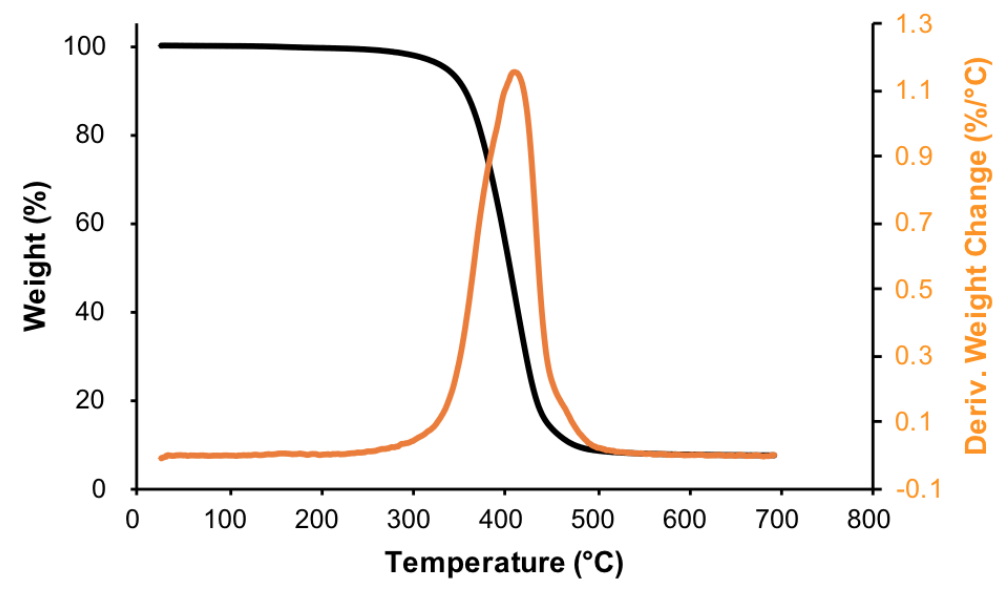

Figure S54. TGA and DTG curves of poly(EtVCP-VE) in Table 2, entry 4 . $T_{\mathrm{d}}$ (10\% weight loss $)=354{ }^{\circ} \mathrm{C} ; T_{\max }=411{ }^{\circ} \mathrm{C}$.

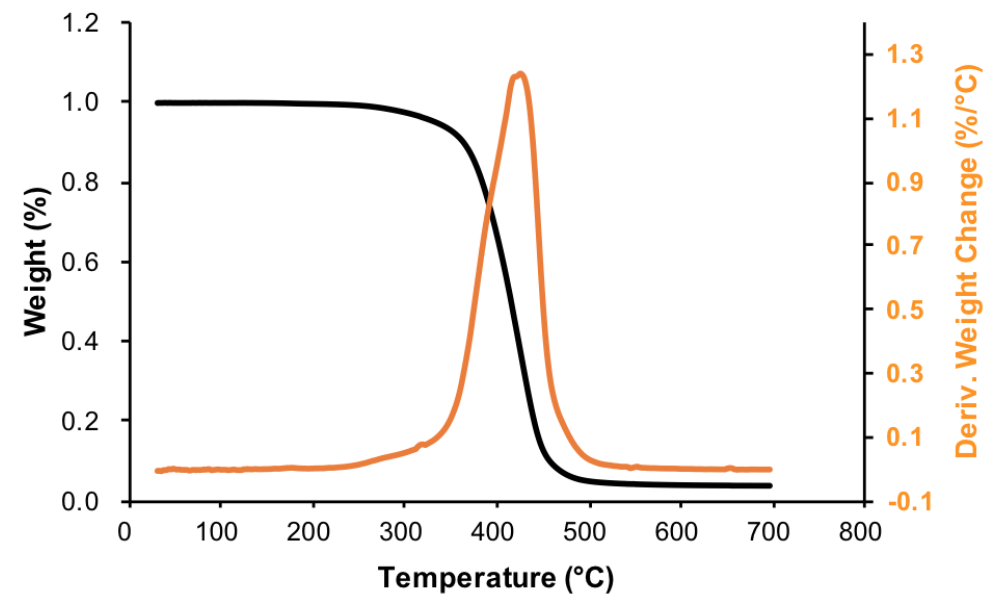

Figure S55. TGA and DTG curves of poly(EtVCP-VE) in Table 3, entry $3 . T_{\mathrm{d}}$ (10\% weight loss $)=363{ }^{\circ} \mathrm{C} ; T_{\max }=425^{\circ} \mathrm{C}$. 


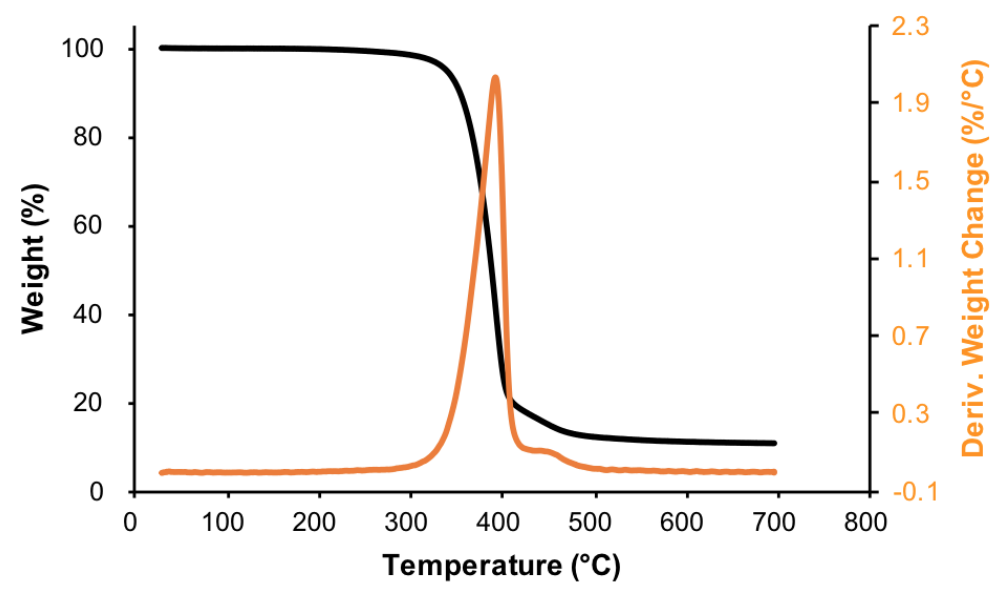

Figure S56. TGA and DTG curves of poly(EtVCP-U) in Table 2, entry $13 . T_{\mathrm{d}}(10 \%$ weight loss $)=354{ }^{\circ} \mathrm{C} ; T_{\max }=393{ }^{\circ} \mathrm{C}$.

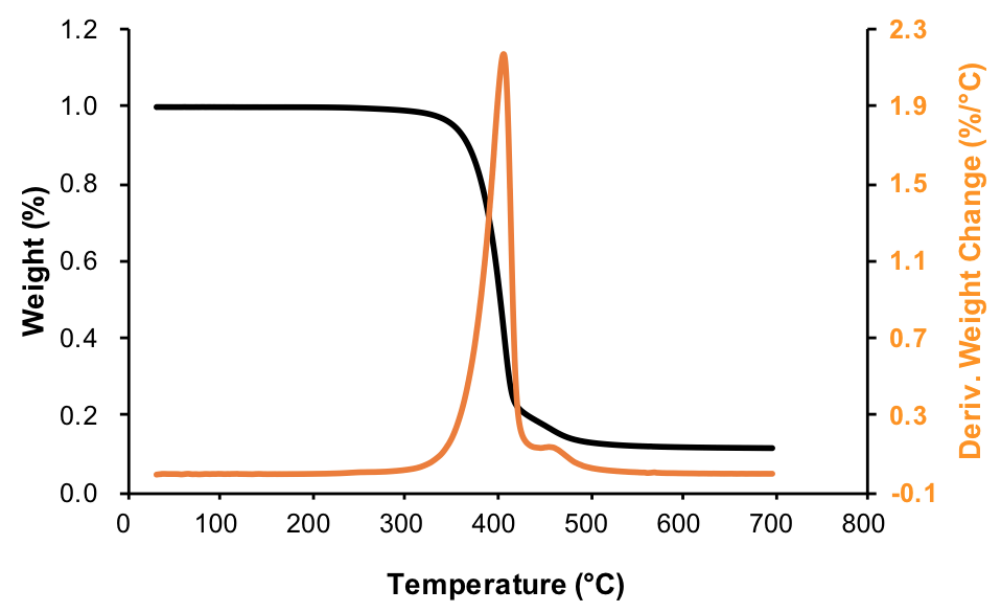

Figure S57. TGA and DTG curves of poly(EtVCP-U) in Table 3, entry 4. $T_{\mathrm{d}}(10 \%$ weight loss $)=367{ }^{\circ} \mathrm{C} ; T_{\max }=405^{\circ} \mathrm{C}$. 


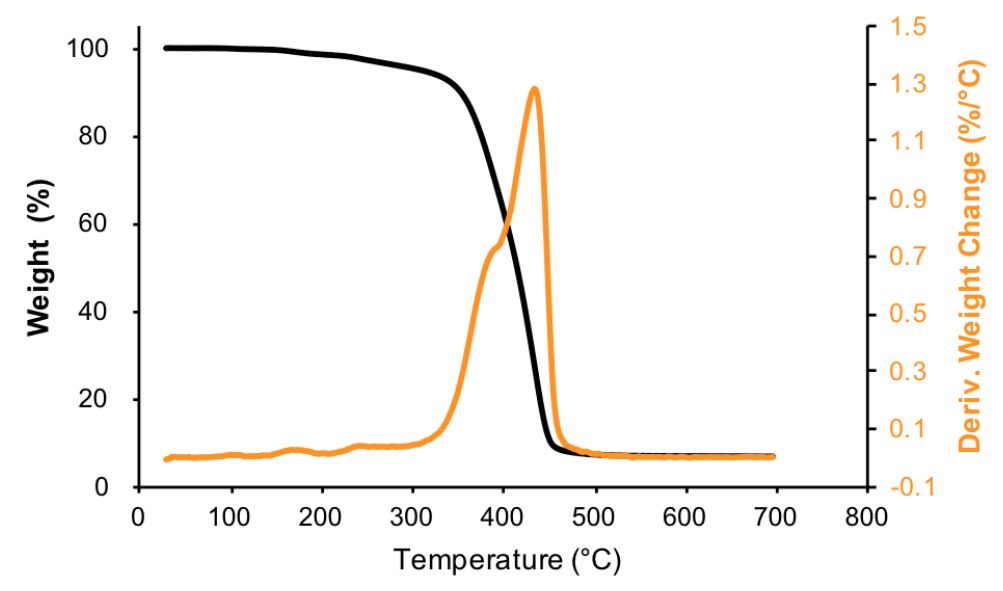

Figure S58. TGA and DTG curves of poly(EtVCP-DA) in Table 2, entry 14. $T_{\mathrm{d}}(10 \%$ weight loss) $=353{ }^{\circ} \mathrm{C} ; T_{\max }=434{ }^{\circ} \mathrm{C}$.

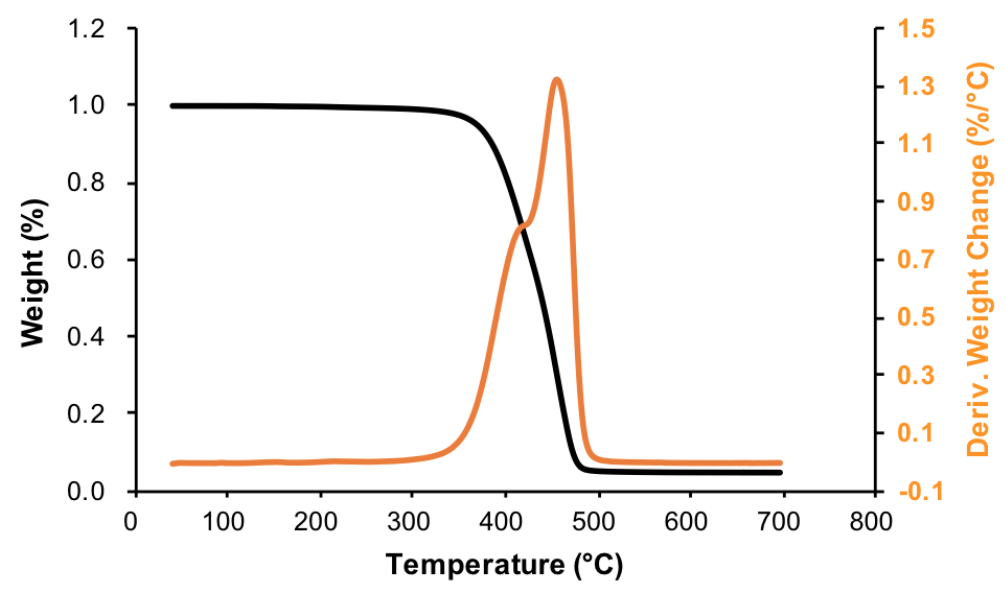

Figure S59. TGA and DTG curves of poly(EtVCP-DA) in Table 3, entry $6 . T_{\mathrm{d}}(10 \%$ weight loss $)=385^{\circ} \mathrm{C} ; T_{\max }=455^{\circ} \mathrm{C}$. 


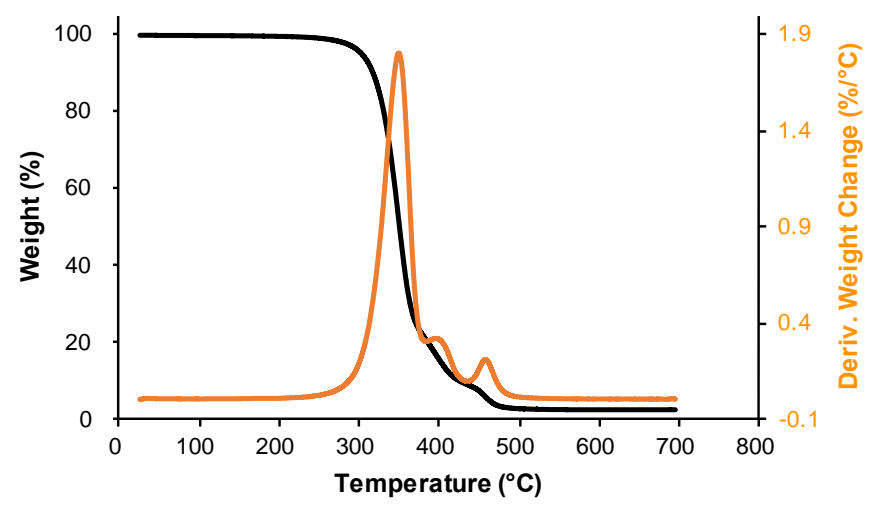

Figure S60. TGA and DTG curves of poly(EtVCP-C1) in Table 2, entry 17. $T_{\mathrm{d}}(10 \%$ weight loss) $=314{ }^{\circ} \mathrm{C} ; T_{\max }=352{ }^{\circ} \mathrm{C}$.

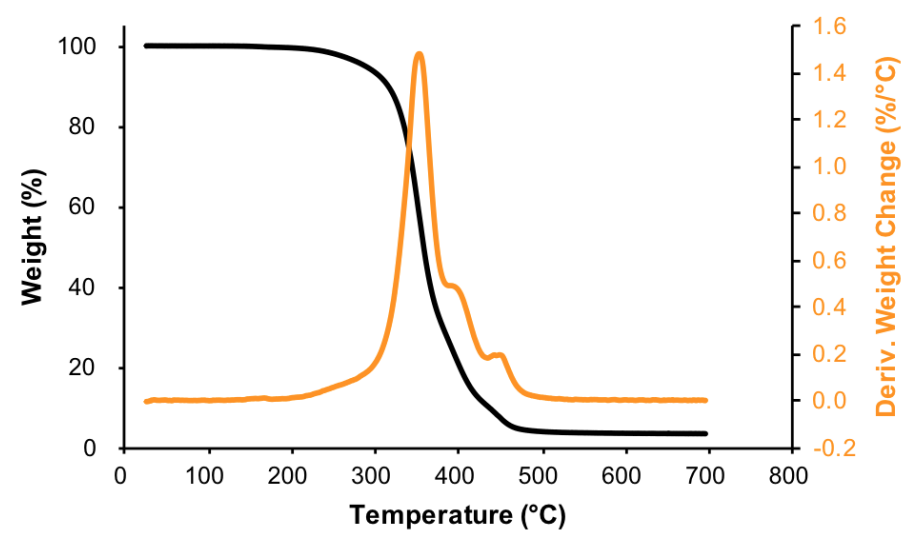

Figure S61. TGA and DTG curves of poly(EtVCP-C) in Table 2, entry $18 . T_{\mathrm{d}}(10 \%$ weight loss $)=314^{\circ} \mathrm{C} ; T_{\max }=352{ }^{\circ} \mathrm{C}$.

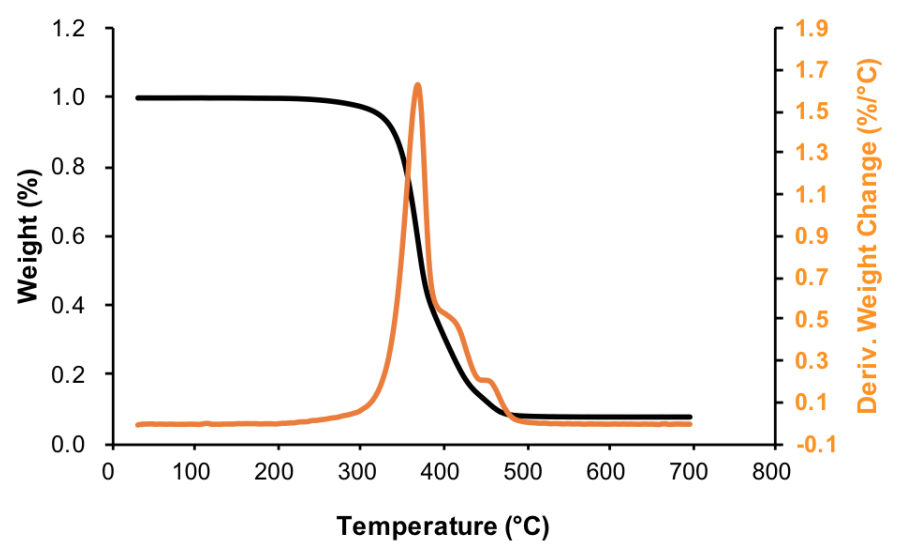

Figure S62. TGA and DTG curves of poly(EtVCP-C2) in Table 3, entry 11. $T_{\mathrm{d}}(10 \%$ weight loss) $=339{ }^{\circ} \mathrm{C} ; T_{\max }=368^{\circ} \mathrm{C}$. 


\section{Polymerization of EtVCP-PDMS}

\subsection{General Experimental Procedure}

General Procedure F: In a N2-filled glove box, M-1 or M-2 (0.20 mmol), 0.10 M DBMM stock solution in EtOAc, and EtOAc were added to a $20 \mathrm{~mL}$ scintillation vial pre-charged with a small magnetic stir bar and PC 1. The vial was then tightly capped and placed into a white-light LED beaker while stirring in the glove box. The temperature was about $28{ }^{\circ} \mathrm{C}$ with a cooling fan. For the analysis of the polymerization at a given time point, a $0.10 \mathrm{~mL}$ aliquot of the reaction mixture was taken via syringe and immediately quenched by injecting into a $1.5 \mathrm{~mL}$ vial containing $\sim 0.70 \mathrm{~mL} \mathrm{CDCl}_{3}$ with 250 ppm butylated hydroxytoluene (BHT). This aliquot was then analyzed via ${ }^{1} \mathrm{H}$ NMR for monomer conversion. The aliquot was then dried under vacuum to remove all volatiles, re-dissolved in THF and passed through a syringe filter for direct analysis by GPC.

General Procedure G: In a N -filled glove box, M-1 or M-2 (0.20 mmol), 0.10 M DBMM stock solution in EtOAc, and EtOAc were added to a $25 \mathrm{~mL}$ Schlenk storage tube precharged with a small magnetic stir bar and PC 1. The Schlenk tube was then tightly capped, taken out of the glove box, then placed into pre-heat oil bath $\left(60{ }^{\circ} \mathrm{C}\right)$ while stirring. A 34 W Kessil blue LED was then immediately turned on. The distance between the Schlenk tube and the blue LED was about $5 \mathrm{~cm}$. The monomer conversion and $S_{\mathrm{L}}$ were determined by crude ${ }^{1} \mathrm{H}-\mathrm{NMR}$ while $M_{\mathrm{n}}$ and $Ð$ of the obtained polymer was determined by the GPC. 


\section{2 ${ }^{1} \mathrm{H}-\mathrm{NMR}$ Spectrum of Brush Poly(EtVCP-PDMS)}

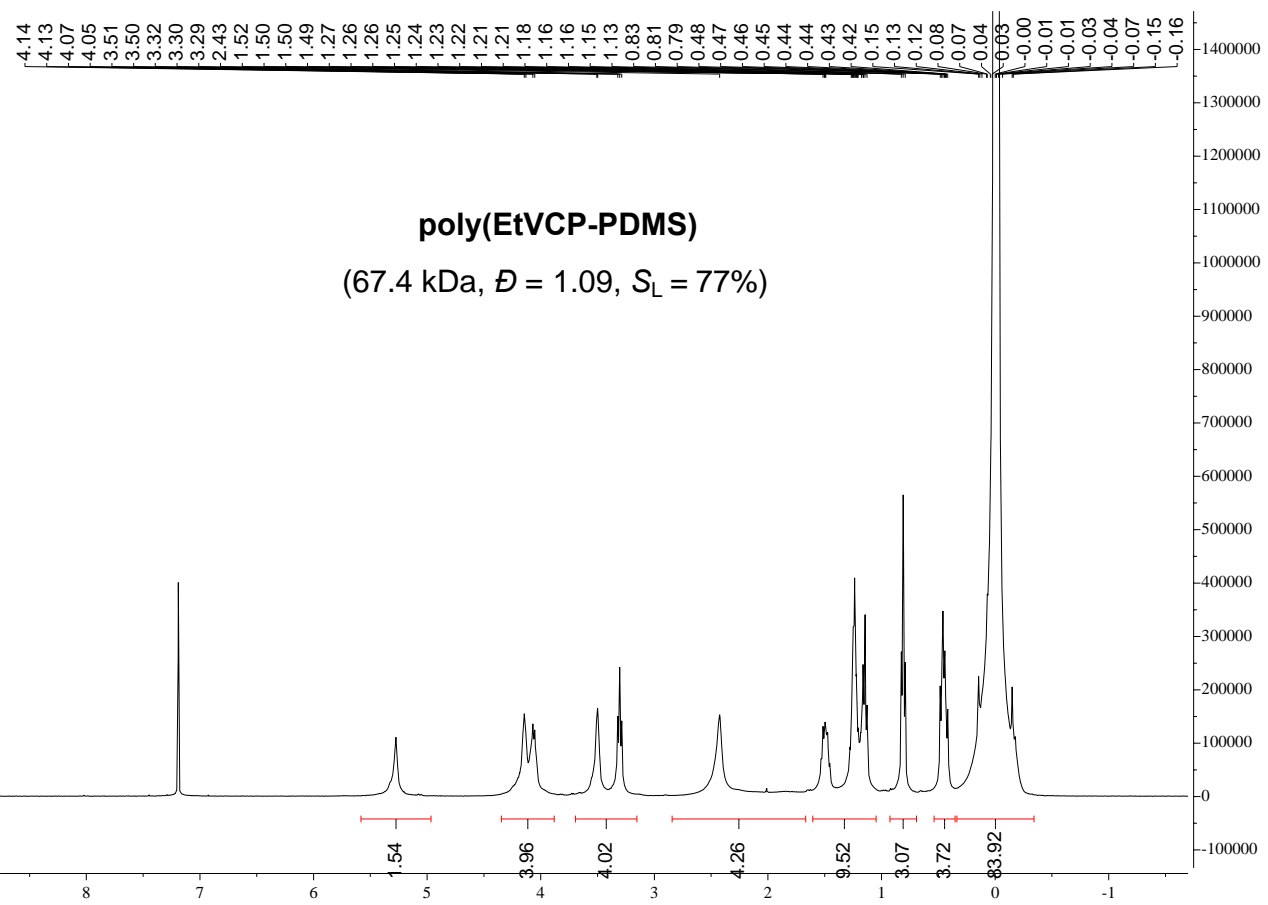

Figure S63. ${ }^{1} \mathrm{H}$-NMR Spectrum of poly(EtVCP-PDMS) in Table 4, entry 1.

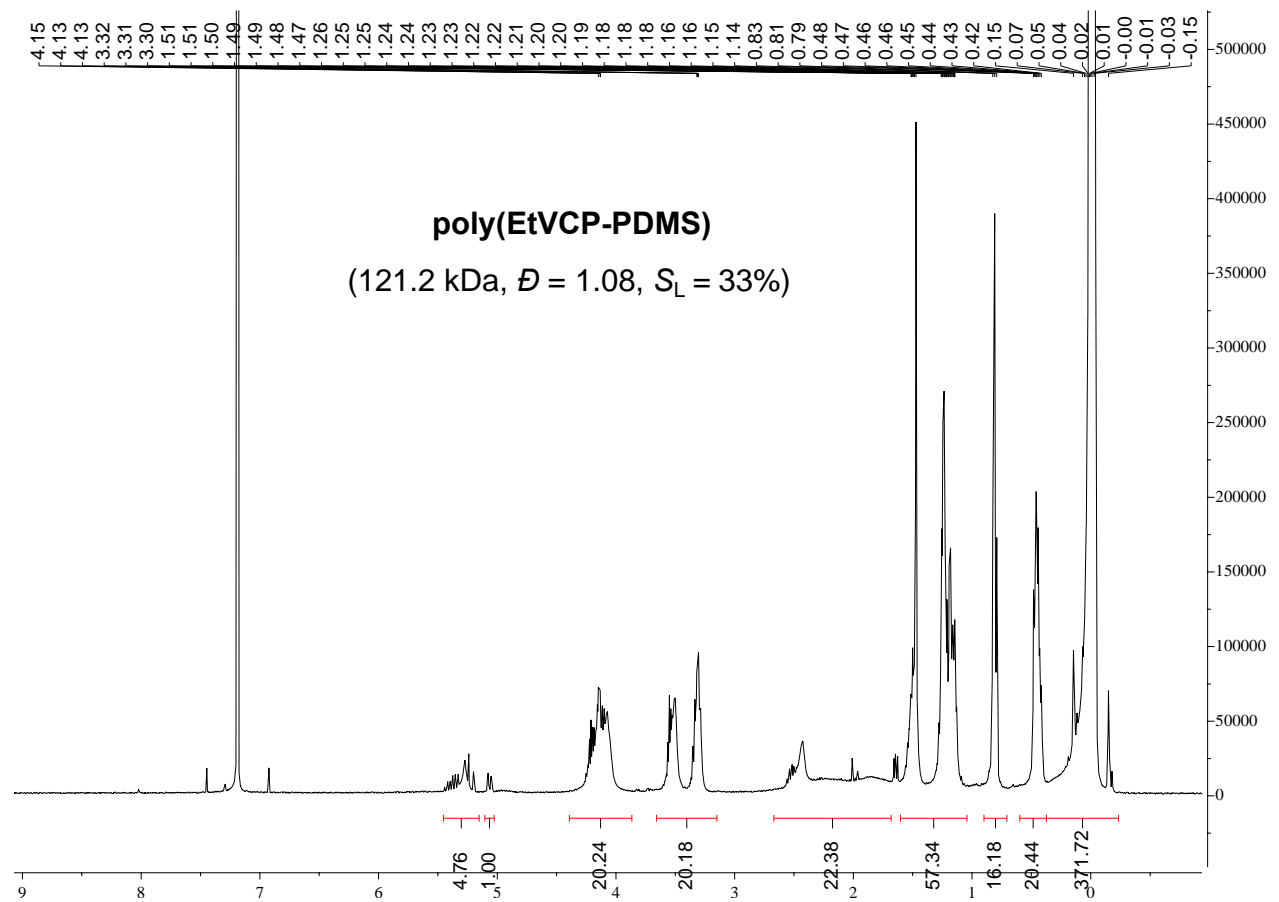

Figure S64. ${ }^{1} \mathrm{H}-\mathrm{NMR}$ Spectrum of poly(EtVCP-PDMS) in Table 4, entry 8. 


\subsection{GPC Traces of Brush Poly(EtVCP-PDMS)}

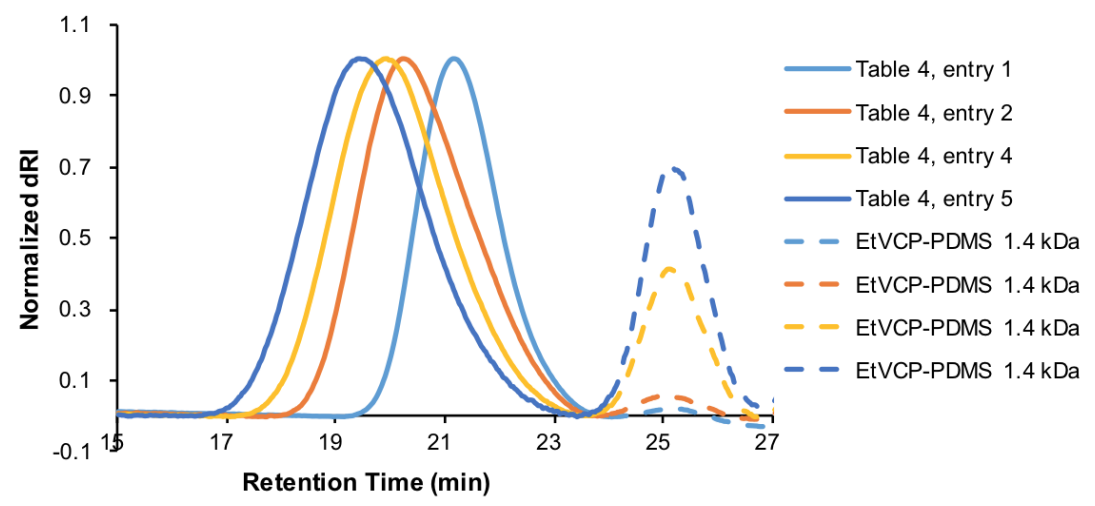

Figure S65. Overlay of GPC traces for brush poly(EtVCP-PDMS) in Table 4, entries 1-2 and 4-5.

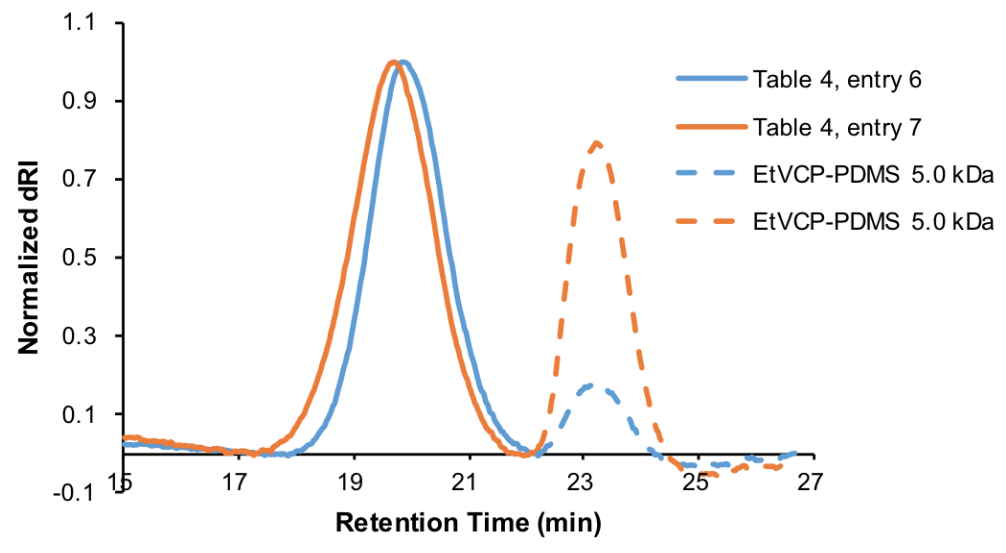

Figure S66. Overlay of GPC traces for brush poly(EtVCP-PDMS) in Table 4, entries 1-2 and 4-5.

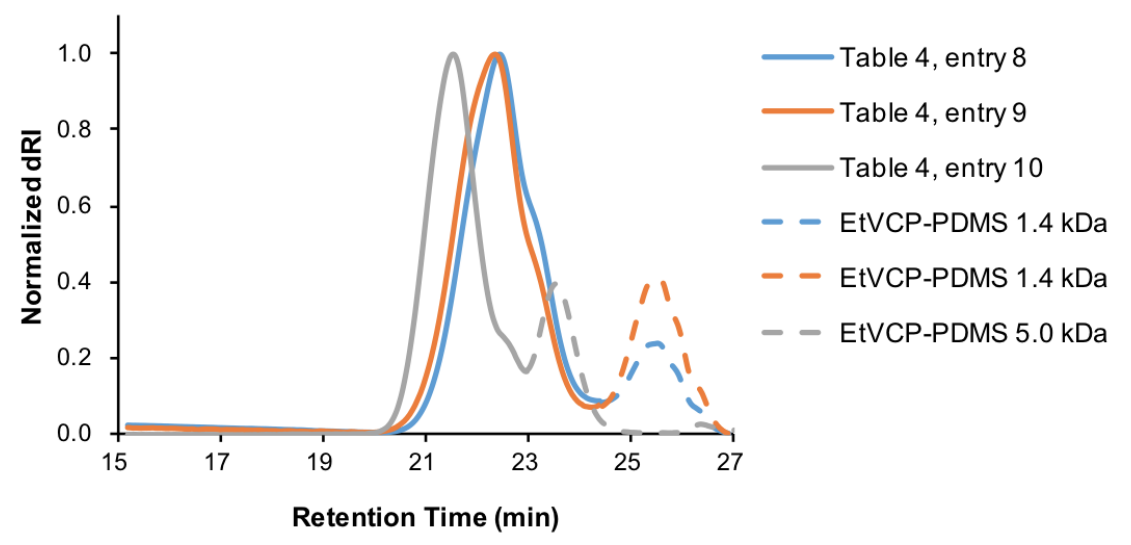

Figure S67. Overlay of GPC traces for brush poly(EtVCP-PDMS) in Table 4, entries 8-10. 


\subsection{DSC and TGA Curves of Brush Poly(EtVCP-PDMS)}

Note: in DSC analysis, no $T_{\mathrm{g}}$ s were observed for poly(EtVCP-PDMS) across the range of $-80{ }^{\circ} \mathrm{C}$ to $200{ }^{\circ} \mathrm{C}$.

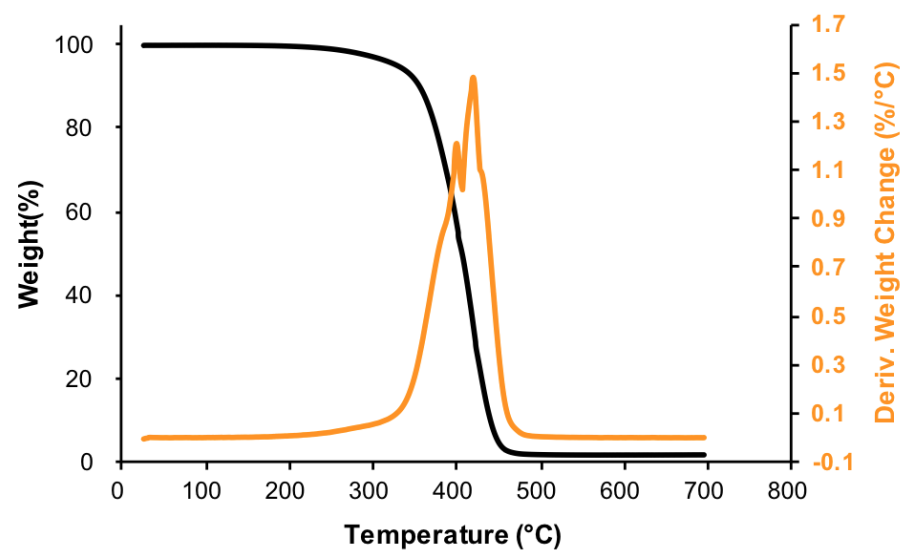

Figure S68. TGA and DTG curves of poly(EtVCP-PDMS) in Table 4, entry 1. $T_{\mathrm{d}}(10 \%$ weight loss) $=357{ }^{\circ} \mathrm{C} ; T_{\max }=422{ }^{\circ} \mathrm{C}$.

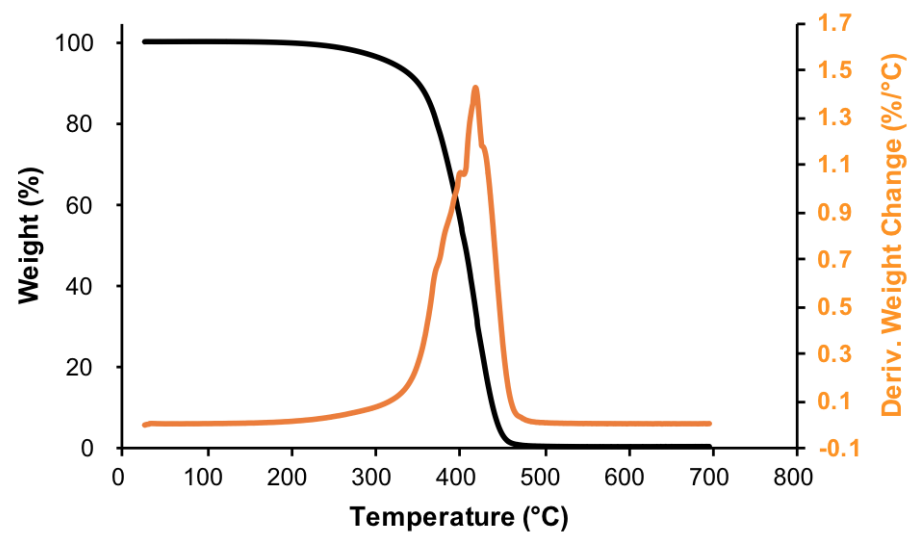

Figure S69. TGA and DTG curves of poly(EtVCP-PDMS) in Table 4, entry 8. $T_{\mathrm{d}}(10 \%$ weight loss) $=350{ }^{\circ} \mathrm{C} ; T_{\max }=419{ }^{\circ} \mathrm{C}$. 


\section{References}

1 Theriot, J. C.; Lim, C.-H.; Yang, H.; Ryan, M. D.; Musgrave, C. B.; Miyake, G. M. Organocatalyzed atom transfer radical polymerization driven by visible light. Science 2016, 352, 1082-1086.

2 Pearson, R. M.; Lim, C.-H.; McCarthy, B. G.; Musgrave, C. B.; Miyake, G. M. Organocatalyzed atom transfer radical polymerization using $\mathrm{N}$-aryl phenoxazines as photoredox catalysts. J. Am. Chem. Soc. 2016, 138, 11399-11407.

3 Casper, P.; Ritter, H. A Novel Liquid-Crystalline Vinylcyclopropane-Derivative Bearing Cholesterol: Synthesis and Polymerization. J. Macromol. Sci., Part A: Pure Appl.Chem. 2003, 40, $107-113$.

4 Emmett, M. R.; Kerr, M. A. Nucleophilic ring opening of cyclopropane hemimalonates using internal Brønsted acid activation. Org. Lett. 2011, 13, 4180-4183.

5 Sekine, M. General method for the preparation of N3- and O4-substituted uridine derivatives by phase-transfer reactions. J. Org. Chem. 1989, 54, 2321-2326.

6 Thommen, C.; Jana, C. K.; Neuburger, M.; Gademann, K. Syntheses of Taiwaniaquinone $\mathrm{F}$ and Taiwaniaquinol $\mathrm{A}$ via an Unusual Remote $\mathrm{C}-\mathrm{H}$ Functionalization. Org. Lett. 2013, 15, 1390-1393. 